\title{
Nonthermal particles and photons in starburst regions and superbubbles
}

\author{
Andrei Bykov
}

Received: date / Accepted: date

\begin{abstract}
Starforming factories in galaxies produce compact clusters and loose associations of young massive stars. Fast radiation-driven winds and supernovae input their huge kinetic power into the interstellar medium in the form of highly supersonic and superalfvenic outflows. Apart from gas heating, collisionless relaxation of fast plasma outflows results in fluctuating magnetic fields and energetic particles. The energetic particles comprise a long-lived component which may contain a sizeable fraction of the kinetic energy released by the winds and supernova ejecta and thus modify the magnetohydrodynamic flows in the systems. We present a concise review of observational data and models of nonthermal emission from starburst galaxies, superbubbles, and compact clusters of massive stars. Efficient mechanisms of particle acceleration and amplification of fluctuating magnetic fields with a wide dynamical range in starburst regions are discussed. Sources of cosmic rays, neutrinos and multi-wavelength nonthermal emission associated with starburst regions including potential galactic "PeVatrons" are reviewed in the global galactic ecology context.
\end{abstract}

Keywords Starburst, supernova remnants, stellar winds, interstellar medium, cosmic rays, high energy neutrinos

\section{Introduction}

Almost eighty years ago W. Baade and F. Zwicky proposed a very profound idea that supernovae are the sources of the bulk of relativistic particles that are detected at the Earth as cosmic rays (CRs). This idea connects the problem of the origin of cosmic rays to the problem of stellar evolution - the crucial phenomenon in physics of galaxies and clusters of galaxies. This connection is not just a one way relationship, as it puts the non-thermal components into the context of interstellar and intergalactic gas dynamics with tight links to cosmic magnetic fields, chemical evolution of matter, star formation $(\mathrm{SF})$ and other phenomena of fundamental

A M Bykov

Ioffe Institute, 194021 St.Petersburg, Russia

E-mail: byk@astro.ioffe.ru 
importance. Indeed, the observed energy density of relativistic particles in the Earth vicinity (about $1.5 \mathrm{eV} \mathrm{cm}^{-3}$ ) is comparable with energy densities of the other major constituents of the interstellar medium (ISM) in the Milky Way. The power of CR sources needed to sustain the observed CR energy density assuming a quasi-steady CR propagation regime and using the observed secondary to primary CRs ratios and anisotropy can be estimated as about $3 \times 10^{38} \mathrm{erg} \mathrm{s}^{-1} \mathrm{kpc}^{-2}$ with the total power $\gtrsim 3 \times 10^{40} \mathrm{erg} \mathrm{s}^{-1}$ (see, e.g., Ginzburg and Syrovatskii 1964 , Berezinskii et al 1990, Strong et al 2007).

The cosmic-ray pressure in violent star forming regions may reach a critical value which is analogous to the well known Eddington limit resulting in large scale outflows in the form of local chimneys or global galactic wind driven by cosmic rays (see e.g. Socrates et al 2008, Salem and Bryan 2014). The CR-driven outflows along with the pressure driven winds (Chevalier and Clegg 1985, Norman and Ikeuchi 1989, Veilleux et al 2005) could affect the star formation rate (see e.g. Bolatto et al 2013, and the references therein).

In this review we briefly outline the basic properties of SF regions and starburst phenomena, then concentrate on particle acceleration and magnetic field amplification processes in their connection to SF, and finally discuss radiation processes and multi-wavelength spectra of nonthermal emission of SF regions.

\section{Star formation in the Milky Way and other galaxies}

Young massive stars are the main acting agents in the chemical and dynamical evolution of galaxies and they are the sources of non-thermal components - cosmic rays and associated magnetic fields. These stars are associated with regions of recent SF activity. The SF is of fundamental importance for galactic ecology. The Milky Way is a mildly-barred, gas-rich spiral galaxy. The Galactic disk contains about $(2-6) \times 10^{9} \mathrm{M}_{\odot}$ of atomic hydrogen $\mathrm{H} \mathrm{I}$ and $(1-3) \times 10^{9} \mathrm{M}_{\odot}$ of molecular hydrogen $\mathrm{H}_{2}$ (see, e.g., Combes 1991, Ferrière 2008). It is characterised by the most active $\mathrm{SF}$ in the Local Group of about $2 \mathrm{M}_{\odot} \mathrm{yr}^{-1}$. The estimations of the rate of gas infall from the Local Group are highly uncertain and range from 0.1 to $1 \mathrm{M}_{\odot} \mathrm{yr}^{-1}$. The mean SF efficiency per giant molecular cloud in the solar vicinity is about $5 \%$ (see, e.g., Molinari et al 2014, for a review).

Recent high resolution multi-wavelength observations and numerical simulations allowed us to look deep into SF though some details of the process are not very clear as of yet. SF is known to be due to gravitational collapse of the dense cores of molecular clouds. Supersonic turbulent gas motions, magnetic fields in the molecular cloud, as well as radiative and mechanical feedback from the formed stars each play a role in this process (e.g., Federrath et al 2011, Hennebelle and Falgarone 2012, Renaud et al 2014).

Magnetic fields may provide a support to low density gas on large scales, while the local gas heating due to radiation feedback from (proto)stars was found to be able to strongly suppress fragmentation on the small scales (see, e.g., Price and Bate 2009). Both effects were predicted to result in rather an inefficient SF process in a molecular cloud, with a star formation rate (SFR) of less than $10 \%$ of the cloud mass for the free-fall time, that roughly corresponds to the observed rate. On the other hand, rather surprisingly, current observations support the empirical star formation laws, which indicate that the area-averaged SFR depends mostly on 


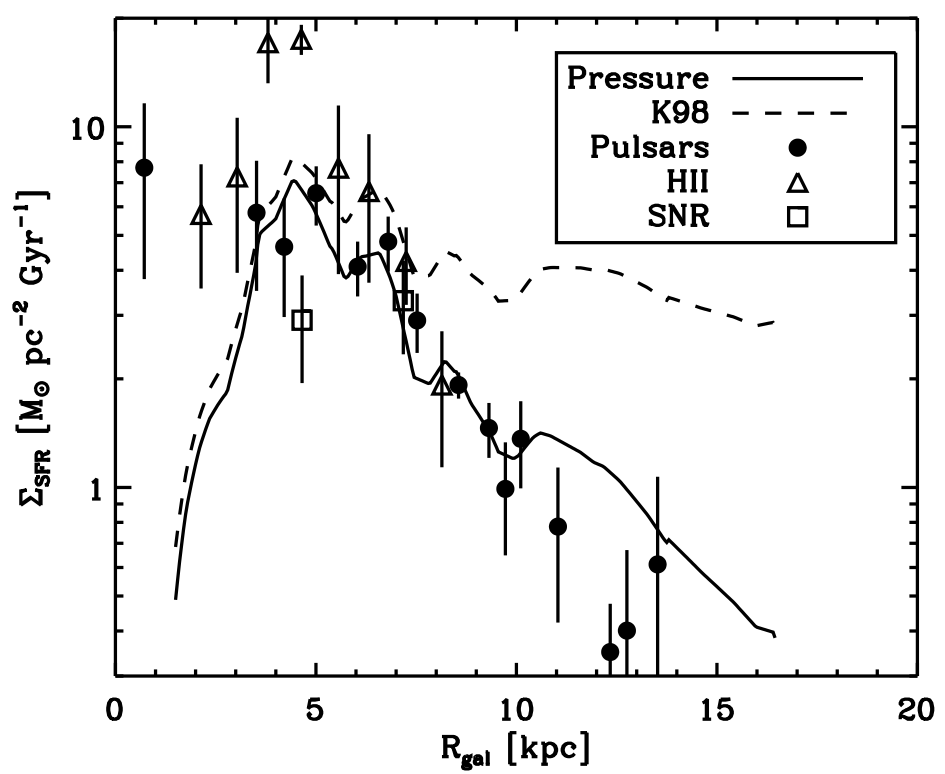

Fig. 1 Star formation (SF) rate as a function of galactocentric radius $\mathrm{R}_{\text {gal }}$ for the Milky Way adopted from Blitz and Rosolowsky (2006). The SF rate values as derived from observations of H II regions by Guesten and Mezger (1982) (triangles), from galactic pulsar population by Lyne et al. (1985) (circles) and from supernova remnants Guibert et al. (1978) (squares). The pressure-based SF prescription by Blitz and Rosolowsky (2006) is given by the solid line to be compared to that of Kennicutt (1998) (dashed line). The pressure formulation predicts not only the shape of the curve for the observed SF rate, but for most of the points, the amplitude as well.

the surface density of molecules (see, e.g., Elmegreen 2012). An analysis of Blitz and Rosolowsky (2006) revealed that the ratio of atomic to molecular gas $R_{\text {mol }}$ in galaxies is determined by hydrostatic pressure of the interstellar gas $P_{\text {ext }}$. They found that $R_{\text {mol }} \propto P_{\text {ext }}^{0.92 \pm 0.10}$, at a given radius in a spiral galaxy and that the relationship between the two holds over 3 orders of magnitude in pressure. Since SF in giant molecular clouds determine the galactic SFR, Blitz and Rosolowsky (2006) proposed a pressure-based prescription for $\mathrm{SF}$ in galaxies that is based on the $R_{\text {mol }}-P_{\text {ext }}$ relation which correctly describes SFR in molecule-poor galaxies (e.g., in the outer regions of large spirals, dwarf galaxies, damped Ly $\alpha$ systems) while for molecule-rich galaxies the prescription has a form similar to that suggested by Kennicutt (1998b). In Figure 1 adopted from Blitz and Rosolowsky (2006), the SFR in the Milky Way is shown as a function of galactocentric radius $\mathrm{R}_{\text {gal }}$. The figure compares the SFR values as estimated from observations of H II regions by Guesten and Mezger (1982), from galactic pulsar population by Lyne et al (1985), and from supernova remnants by Guibert et al (1978) with results of the pressure-based star formation prescription by Blitz and Rosolowsky (2006) given by the solid line and that of Kennicutt (1998b). The pressure formulation not only predicts the shape of the curve for the SFR observed in the Milky Way, but also accounts for its amplitude. It may naturally explain the high SFRs in some 
galactic merger events. In a recent parsec-resolution simulations of galaxy mergers Renaud et al (2014) demonstrated the triggering of starbursts. They showed that a gravitational encounter between two galaxies enhances tidal compression and compressive turbulent mode thus leading to high SF activity.

The empirical SF law found in galaxy disks suggested an approximately linear relationship between the SFR per unit area and the molecular cloud mass per unit area with a timescale for molecular gas conversion into stars to be about a few Gyr if the gas surface density is $\gtrsim 10 \mathrm{M}_{\odot} \mathrm{kpc}^{-2}$ (see, e.g., Elmegreen 2011a c, for a review). On the other hand, the depletion time, which is usually defined as the ratio of the gas mass to the SFR, is about 100 Gyr in the outer parts of galaxies and in dwarf irregular galaxies with the gas surface density below $10 \mathrm{M} \odot \mathrm{kpc}^{-2}$ where the disk is dominated by atomic hydrogen. Following Krumholz and McKee (2005) it is instructive to introduce $\epsilon_{\mathrm{ff}}$ - the ratio of the free fall time of self-gravitating gas in molecular clouds to the gas depletion time. Observations compiled in Figure 4 of Krumholz $(2014)$ suggested $\epsilon_{\mathrm{ff}} \approx 0.01$ as the universal value for galaxies in the local Universe. The complex process of matter cycling from the ISM gas into stars with the return of metal rich material with high energy stellar ejecta is the driver of galactic evolution through cosmic time (see for a recent review Draine 2011, Molinari et al 2014, Krumholz 2014, and references therein). Cosmic gas accretion by galaxies affects global SF. In particular, the relationship between stellar mass, metallicity, and the SFR (the fundamental metallicity relationship) observed in large samples of SF galaxies can be explained by metal-poor gas accretion which is especially important at high redshifts as argued by Sanchez Almeida et al (2014). They discussed the global SF history of the universe and the history of gas around galaxies as inferred from the observed absorption features in the spectra of background sources.

Synthesis of elements and dust, chemical evolution of galaxies, large scale motions, cosmic rays, and magnetic fields in ISM are the integral parts of the global star formation process. While multi-wavelength observations have yielded a lot of information and phenomenological models exist, a comprehensive, quantitative theory of the process is still to be constructed. A challenging issue is the feedback from newborn clustered stars on the SF environment in molecular clouds that limits the rate and efficiency of SF.

Observations support general picture of formation of highly structured, clumped dense molecular clouds from diffuse tenuous ISM gas resulting in SF with subsequent feedback processes due to radiation and kinetic energy release from $\mathrm{OB}$ associations and compact clusters of young massive stars. Formation of dense cold clouds in the ISM is likely a result of multi-fluid gravitational instabilities of gas shocked by by spiral density waves. The gas motions accelerated by the radiation and winds of young hot stars clear out cavities in the intercloud matter and accumulate gas on the periphery of the cavities.

The rate at which the gas is converted into stars is controlled locally by the radiation of young stars, turbulence, magnetic fields, and feedback by winds of massive stars and supernovae with the emergence of superbubbles though largescale galactic structures such as the spiral arms and the central bar (e.g., Price and Bate 2009, Elmegreen 2012, Krumholz et al 2014, Krause et al 2014). The relative role and the links between the processes listed above are still not very clear, but the pressure is an important factor. Stellar radiation and evolution of supernovae at later stages are the sources of gas momentum in SF regions, which is relaxed in 
part through shocks into high pressure gas bubbles and superbubbles. The bubbles may trigger sequential SF with locally increased SFR (Elmegreen and Lada 1977).

A statistical study of young stellar objects in the vicinity of 322 mid-infrared bubbles detected by Spitzer (Thompson et al 2012) revealed that the fraction of massive stars in the Milky Way formed by the triggering process is possibly between 14 and $30 \%$. Fast shocks of velocity $v_{\mathrm{sh}}$ of a few thousands $\mathrm{km} \mathrm{s}^{-1}$ produce hot gas with temperature well above $10^{6} \mathrm{~K}$, which slowly cools thus providing long lived high pressure bubbles. The shocks are also efficiently producing nonthermal pressure components consisting from highly amplified fluctuating magnetic fields and relativistic particles with partial pressure $P_{\mathrm{nt}}$, which may reach a sizeable fraction $\eta \lesssim 0.5$ of the shock ram pressure (i.e., $P_{\mathrm{nt}}=\eta \rho v_{\mathrm{sh}}^{2} / 2$ ). The nonthermal components may affect the ionization and sources of turbulence in molecular clouds. In this review we discuss the origins and major properties of the nonthermal components in SF regions.

The estimated global SFR in different galaxies ranges by many orders of magnitude from less than $10^{-3} \mathrm{M} \odot \mathrm{yr}^{-1}$ in some galaxies with very inefficient star formation to about $10^{4} \mathrm{M}_{\odot} \mathrm{yr}^{-1}$ in the so-called ultra luminous infrared galaxies (ULIRGs) which show far-infrared (FIR) luminosities above $10^{12} \mathrm{~L} \odot$. The average SFR has a clear trend with the galaxy Hubble type, though a strong dispersion is present in SFR distribulions of galaxies of the same type Kennicutt (1998a). The SFR variations are time dependent and can be caused by the high dispersion in gas content, galactic interactions and activity of galactic nuclei.

While the low SFR at the level below $10^{-2} \mathrm{M}_{\odot} \mathrm{yr}^{-1}$ was revealed by deep Fabry-Perot $\mathrm{H}_{\alpha}$ imaging in some $\mathrm{S} 0$ type galaxies, some other HI-rich S0 galaxies as well as other spirals of types Sa and later demonstrated somewhat higher SF activity. Observations with Spitzer and Herschel revealed that SFR strongly increases with redshift for luminous infrared galaxies. Use of mid-IR spectroscopy allowed to separate the signal from dust heated by massive stars from that heated by AGNs. At low redshifts ULIRGs are mainly characterized by circumnuclear type of starburst activity initiated by mergers while the high redshift population of ULIRGs is dominated by the galaxies with extended SF regions Kennicutt and Evans (see, e.g., 2012, and references therein). In this review we mainly focus on active SF regions and starburst phenomena.

\section{Active starforming regions and starburst galaxies}

Possible criteria of the starburst following are a high SFR with gas-consumption timescales of less than $1 \mathrm{Gyr}$ and SFR surface density averaged over the disk above $0.1 \mathrm{M}_{\odot} \mathrm{yr}^{-1} \mathrm{kpc}^{-2}$ (Kennicutt and Evans 2012). The high SFR can not be sustained for longer than a small fraction (e.g., less than 10\%) of the Hubble time. Starburst regions with SFR of about $100 \mathrm{M}_{\odot} \mathrm{yr}^{-1}$ are not active for much longer than 100 Myr. The typical observational appearances of the starburst in either nucleus or active regions of a galaxy are (i) the large ratio of the infrared (IR) to the bolometric luminosity, which is due to re-radiation of the luminous emission of hot young stars absorbed by the surrounding dust in the IR band, (ii) high luminosity of Balmer-line emission with large equivalent widths, or (iii) strong radio continuum emission. 
A number of starburst galaxies are already well known, the most studied among these being the nearby Irr2 type galaxy Messier 82, NGC 253 (a barred Sc galaxy), and Arp 220 (likely a merger). Starbursts are usual phenomena in interacting and merging galaxies. The Antennae Galaxies are a pair of interacting starburst galaxies NGC 4038/NGC 4039 located in the Corvus constellation. The observed ongoing phase of starburst in NGC 4038/NGC 4039 (see e.g. Whitmore and Schweizer 1995. Whitmore et al 2010) is supporting the suggestions that the dense high pressure regions in merging galaxies are the regions with high SFR (see e.g. Blitz and Rosolowsky 2006, Renaud et al 2014).

The observed FIR - radio correlation in SF galaxies indicates that massive stars are the energy source for both FIR and nonthermal radio emission (see e.g. de Jong et al 1985, Helou et al 1985, Voelk 1989, Condon 1992, Hartquist et al 2007, Schleicher and Beck 2013. Zweibel 2013). FIR in starforming galaxies is mostly produced by the dust heated by the radiation of stars more massive than $5 \mathrm{M}_{\odot}$, and $\mathrm{L}_{60 \mu \mathrm{m}}$ characteristic luminosity at $60 \mu \mathrm{m}$ is connected to the SFR as

$$
\operatorname{SFR}\left(M>5 \mathrm{M}_{\odot}\right)=\frac{\mathrm{L}_{60 \mu \mathrm{m}}}{5.110^{23} W H z^{-1}} \mathrm{M}_{\odot} \mathrm{yr}^{-1},
$$

where $\operatorname{SFR}(M>5 \mathrm{M} \odot)$ is the rate of the formation of stars heavier than $5 \mathrm{M}_{\odot}$ (see, e.g., Cram et al 1998).

Starburst galaxies are known to have high supernova rates in the active SF regions. Radio and IR observations of M82 (see Unger et al 1984, Kronberg et al 1985, Voelk 1989, Condon 1992, Huang et al 1994,|Pedlar and Wills 2007) revealed many supernova remnants (SNRs). The radio luminosity of an SF regions is assumed to be proportional to the recent $\mathrm{SF}$ rate and is thought to be connected to $S F R\left(M>5 \mathrm{M}_{\odot}\right)$. With a particular model of the origin of thermal and nonthermal radio continuum radiation it is possible to disentangle the nonthermal radio luminosity (e.g., Biermann 1976, Condon 1992, Cram et al 1998) and thus obtain the SFR as

$$
\operatorname{SFR}\left(M>5 \mathrm{M}_{\odot}\right)=\frac{\mathrm{L}_{1.4 \mathrm{GHz}}}{4.010^{20} W H z^{-1}} \mathrm{M}_{\odot} \mathrm{yr}^{-1} .
$$

\subsection{Initial mass functions of clusters and stars}

The star formation activity results in some hierarchical structure with both spatial and temporal correlations (see, e.g., Elmegreen 2011b), which is important for ISM ecology and evolution (McCray and Kafatos 1987, Mac Low and McCray 1988, Norman and Ikeuchi 1989, Heiles 1990, 2001). Young massive SF regions are often clumped Lada and Lada (2003), Portegies Zwart et al (2010). Active SF regions may consist of a number of clusters and extended populations of massive stars. On the other hand, some of the large SF regions are loose OB star associations like Cyg OB2, where no signatures of dense star clusters was found (see e.g. Wright et al 2014). It was argued by Tutukov (1978), Lada and Lada (2003) that about $90 \%$ of the clusters disperse within about 10 Myr.

A fundamental question is why and how the stars that are initially born in high density, highly-clustered SF regions eventually form a field population, where the majority of the stars are not bound to any structure smaller than the galaxy as a 
whole, while some minor population still resides in gravitationally-bound clusters with an initial mass function (IMF) at birth $d N / d M \propto M^{-\beta} \exp \left(-M / M_{*}\right)$ with $\beta \approx 2$ (e.g. Krumholz 2014). Observations of young compact clusters indicate that the characteristic mass $M_{*}$ is about $2 \times 10^{5} \mathrm{M}_{\odot}$ in the Milky Way type galaxies, and it is above $10^{6} \mathrm{M}_{\odot}$ in luminous infrared starburst galaxies. It was suggested that young massive clusters of mass well below $10^{6} \mathrm{M}_{\odot}$ may dissolve eventually into unbound field star populations due to their initial gas expulsion and some feedback effects. The most massive young clusters similar to those found in the Antennae galaxies have expected lifetimes of about the Hubble time and may evolve into old massive clusters, which are observed in the Milky Way and other galaxies as globular clusters (see Portegies Zwart et al 2010, and the references therein).

The present day mass functions of the globular clusters are different from the IMF of young stellar clusters discussed above. They are peaked or bell-shaped (for logarithmic mass bins) with the characteristic mass at about $2 \times 10^{5} \mathrm{M}_{\odot}$ which may be either due to evolution effects that affect the IMF or to the initial form of IMF (see Portegies Zwart et al 2010, for a discussion). For a long time globular clusters have been used as natural laboratories for studies of stellar evolution, relaxation of bound N-body systems, and stellar encounters which are frequent in the dense central regions of the globular clusters and may result in formation of a rich sample of exotic objects of various nature (see, e.g., Meylan and Heggie 1997).

Recent investigations revealed a number of new features of these objects. It was established that, contrary to a long-standing paradigm stating that the constituent stars of a globular cluster are born at the same time and made of the same material, it has recently become clear that different stellar populations are present there (see e.g. Anderson et al 2009). At least two different stellar populations were distinguished in 47 Tuc (NGC 104) by Dobrovolskas et al (2014) with small variation in the iron-peak and neutron capture elements but different abundances of proton-capture and alpha-elements. This suggests that a population of the cluster stars exists, which has most likely been formed from the gas enriched by the stars of the previous generation.

The form of the star IMF is of fundamental importance for studies of galaxy evolution. Observations are consistent with the power law $d N / d M \propto M^{-\alpha}$ where Salpeter $(1955)$ deduced $\alpha \approx 2.35$ valid for $0.4 \lesssim M / \mathrm{M}_{\odot} \lesssim 10$. Kroupa $(2001)$ proposed a three power-law IMF representation for Galactic field stars IMF (which is in average populated by 5-Gyr-old stars) with two characteristic mass values at 0.08 and $0.5 \mathrm{M}_{\odot}$. Namely, for the low mass end $0.08 \lesssim M / \mathrm{M}_{\odot} \lesssim 0.5$ the IMF index is $\alpha=1.3$, at $M / \mathrm{M}_{\odot} \leq 0.08$, it is flatter with $\alpha=0.3$, while in Salpeter's regime $M / \mathrm{M}_{\odot} \geq 0.5$ the IMF index is $\alpha=2.3$. Note, that if $\alpha=2.0$ then equal masses would be present in every star mass decade.

The problem of universality of the IMF was examined with numerous observational studies and models (see, e.g., Salpeter 1955, Scalo 1986, Massey 1998, Chabrier and Baraffe 2000, Kroupa 2002, Bonnell et al 2007, Andrews et al 2013). A critical review of both the resolved and the integrated properties of stellar populations in galaxies by Bastian et al (2010) concluded that the IMF is consistent with the gross of the available data and suggested no noticeable variations of the IMF over much of cosmic time. The apparent universality of the stellar IMF is non trivial given the strong variance of the conditions in SF regions in the galaxies. 
Star formation models are connecting the origin of the low-mass stars and brown dwarfs to fragmentation of dense filaments and disks, while the continued accretion in a clustered environment limited by the radiation pressure is the likely mechanism for the high mass SF (see, e.g., Bonnell et al 2007, and references therein). The maximal stellar mass in the IMF is likely governed by the feedback processes such as radiation pressure and ionization, though some other factors may be important as well. Observational estimations range from 150 to $300 \mathrm{M}_{\odot}$ with large uncertainties due to unresolved multiplicity, NIR magnitude-mass relation and some other factors (see, e.g., Tan et al 2014, for a recent discussion). The most massive star reported by now is R136a1 with an estimated mass substantially exceeding $150 \mathrm{M}_{\odot}$ (Crowther et al 2010). This Wolf-Rayet star resides in a super star cluster R136 located in the 30 Doradus region of the Large Magellanic Cloud (LMC).

The issue of a possible dependence of the IMF upper end of star mass $m_{\max }$ on the total stellar birth mass of the embedded star cluster $M_{e c l}$ is under discussion (see, e.g., Weidner et al 2010b, Andrews et al 2013, Weidner et al 2014). If there is no dependence of the maximum star mass on the cluster mass, i.e., the IMF is stochastically populated, then one can expect that a $10^{5} \mathrm{M}_{\odot}$ cluster should contain - in statistical sense - the stars of the same masses as a hundred of $10^{3} \mathrm{M}_{\odot}$ clusters. However, if there is a positive correlation between the maximal stellar mass and the cluster mass as proposed by Weidner et al (2010b) then no stars heavier then about $35 \mathrm{M}_{\odot}$ are expected in clusters of $10^{3} \mathrm{M}_{\odot}$. The selection criteria used by Weidner et al (2010b) to claim the presence of the $m_{\max }-M_{e c l}$ correlation is based on counting of the resolved very young stellar populations of age younger than 4 Myr with no SNRs in the cluster. It was also suggested by Weidner et al (2011)

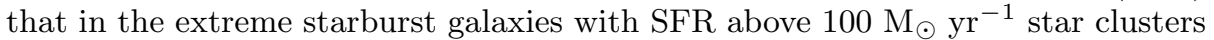
with masses $10^{6} \mathrm{M}_{\odot}$ may have the top-heavy stellar IMF with the slopes of high mass star distributions flatter than the standard index of 2.35 observed in the young massive clusters of mass below $2 \times 10^{5} \mathrm{M}_{\odot}$. However, Andrews et al (2013) used multi-wavelength Hubble Space Telescope WFC3 camera data to measure the production rate of the ionizing photons in unresolved stellar clusters of age below $8 \mathrm{Myr}$ in the nearby irregular galaxy NGC 4214. With this approach they did not find any dependence of the maximum stellar mass on the cluster mass and concluded that within the uncertainties the upper end of the stellar IMF appears to be universal in NGC 4214, thus requiring more observational studies of the existence of the $m_{\max }-M_{e c l}$ correlation.

\subsection{Supernovae in starburst regions}

Assuming that all stars which are more massive than $6.7 \mathrm{M}_{\odot}$ become radio emitting supernovae Condon (1992) estimated the supernova rate in SF regions as

$$
\nu_{\mathrm{SN}}=0.041 \frac{S F R\left(M>5 \mathrm{M}_{\odot}\right)}{\mathrm{M}_{\odot} \mathrm{yr}^{-1}} \mathrm{yr}^{-1} .
$$

From observations of radio SNRs Huang et al (1994) estimated the suprnova rate in M82 as $0.11 \pm 0.05 \mathrm{yr}^{-1}$. At the same time, Smith et al (1998) resolved the radio nucleus of Arp 220 and found many radio-emitting point sources, which were 
identified as SNRs. They estimated the SFR in Arp 220 as about $50-100 \mathrm{M}_{\odot} \mathrm{yr}^{-1}$ and estimated he supernova rate in the galaxy as 1.75-3.5 $\mathrm{yr}^{-1}$. Another nearby nuclear starburst galaxy NGC 253 is a barred Sc spiral galaxy in the Sculptor group located at a distance of about $3.5 \mathrm{Mpc}$ where one arcsecond corresponds to about 17 parsecs. Recent radio observations at $2.3 \mathrm{GHz}$ performed with the Australian Long Baseline Array in a combination with the Jansky Very Large Array were used by Rampadarath et al (2014) for multi-epoch studies of SNRs in NGC 253. Note that to estimate the value of $S F R\left(M>5 \mathrm{M}_{\odot}\right)$ with Eqs. (3) (3.2) it is enough to measure just a single parameter. This helps to constrain the supernova rate in a number of particular cases.

It is very important to find independent ways to estimate both the SFRs and the supernova rates. Hubble Space Telescope NICMOS [Fe II] $1.26 \mu \mathrm{m}$ line detection was proposed by Alonso-Herrero et al (2003) to independently measure supernova rate in M82. The authors suggested that the [Fe II] lines may be produced by an SNR population of ages up $10^{4}$ years, while radio SNRs are a younger population of a few hundred years old. Rosenberg et al (2012) found a nearly linear relationship between the $1.26 \mu \mathrm{m}$ [Fe II] luminosity and the supernova rate in a sample of 11 nearby starburst galaxy centers. This relationship is valid for normal $\mathrm{SF}$ galaxies but does not hold for extreme ultraluminous galaxies. The authors pointed out that a somewhat excessive supernova rate measured in NGC 6240 and Arp 220 compared to radio supernova rate estimatio ns is likely caused by merger shocks that are overexciting the $1.26 \mu \mathrm{m}$ [Fe II] emission line in addition to the excitations produced by SNRs themselves.

Multi-wavelength observations reviewed by Hartquist et al (2007) are consistent with the presence of a central SF region in M82 of about $0.7 \mathrm{kpc}$ scale size with $\operatorname{SFR}\left(M>5 \mathrm{M}_{\odot}\right) \sim 2 \mathrm{M}_{\odot} \mathrm{yr}^{-1}$ and the total $\mathrm{SFR}$ of about $10 \mathrm{M}_{\odot} \mathrm{yr}^{-1}$ within the central $0.3 \mathrm{kpc}$, which is 3 times higher than the SFR of the whole Milky Way. The ultraluminous merger Arp 220 (IC 4553/4) with IR luminosity of about $10^{12.2} \mathrm{~L} \odot$ in the $8-1,000 \mu \mathrm{m}$ band is located at about $75 \mathrm{Mpc}$ from the Sun. Since $1^{\prime \prime}$ roughly corresponds to $361 \mathrm{pc}$ at the estimated distance of Arp 220, very long baseline interferometry images at $2 \mathrm{~cm}$ and $3.6 \mathrm{~cm}$ wavelength were used to study compact radio continuum sources in this galaxy (see, e.g., Batejat et al 2011). The authors resolved a number of SNRs of diameters above $0.27 \mathrm{pc}$, whose radio luminosities are consistent with the standard diameter - surface brightness relation. The ratio of magnetic field energy density to that of relativistic particles was estimated to be about $1 \%$ and magnetic field strengths were found to be about $15-50 \mu \mathrm{G}$ in the SNR shells, while an upper limit of $7 \mu \mathrm{G}$ was suggested for the ISM magnetic field in Arp 220. Submillimeter Array imaging of Arp 220 by Sakamoto et al (2008) in the $860 \mu \mathrm{m}$ continuum and $\mathrm{CO}$ emission line reached a resolution of $0.23^{\prime \prime}(80 \mathrm{pc})$ and $0.33^{\prime \prime}(120 \mathrm{pc})$, respectively. The submillimeter continuum emission dominated by emission of heated dust morphologically agrees with the distribution of radio supernovae in the eastern nucleus, while in the bright western nucleus the dust emission is more compact than the supernova distribution. This possibly indicates that the dust heating in the western part of the galaxy is mainly due to the activity of the nucleus (AGN), though a presence of super star cluster can not be excluded yet. AGNs themselves may seriously affect the SF phenomena.

High resolution near IR adaptive optics imaging of the central 100 pc region in the Seyfert I NGC 2110 galaxy with the Keck OSIRIS instrument (Durré and 
Mould 2014) revealed a number of star clusters in the central $90 \times 35$ parsec bar where $[\overline{F e}$ II] emission indicated the shocked material. Each of these clusters is a few times brighter than the Arches cluster in the Galactic Center region of the Milky Way. The SFR in the central core of NGC 2110 is about $0.3 \mathrm{M}_{\odot} \mathrm{yr}^{-1}$. If most of the energy input from supernova and stellar winds in the localized SF regions is thermalized, a strong wind can be driven out of an active region. Chevalier and Clegg (1985) presented a very useful analytical solution for the wind which is driven from a region of a uniform mass and energy deposition. The solution is parameterized by the uniform mass and energy input rates within a SF region of a given radius $R$, which was assumed to be about 200 pc for a starburst galaxy M82. The non-thermal components including magnetic fields and relativistic particles which we shall discuss below may affect the wind properties in a number of aspects.

TeV range gamma-ray photons were detected from NGC 253 with the H.E.S.S. ground-based Cherenkov imaging atmospheric telescope (see, e.g., Acero et al 2009, Abramowski et al 2012b). GeV regime emission was detected with the Fermi Large Area Telescope from the starburst galaxies M82, NGC 253, NGC 1068, NGC 4945 (see, e.g., Abdo et al 2010a, Ackermann et al 2012) with gamma-ray luminosity ranging from $10^{39}$ to $10^{41} \mathrm{erg} \mathrm{s}^{-1}$. In Fig. 2 adopted from Ackermann et al (2012) gamma-ray luminosities of starburst galaxies are shown in comparison with the Local Group sources. Depending on the particular mechanism of emission of the accelerated particles the measured gamma-ray luminosity may constrain the energy content in the non-thermal components, i.e., relativistic particles and magnetic fields (see, e.g., Ohm 2012, Torres and Reimer 2013, Lacki| 2013,| Martin 2014, Yoast-Hull et al 2014). For instance, Lacki et al (2011) argued that if the gammarays observed from M82 and NGC 253 are of hadronic origin, i.e., they come from pion decays following inelastic collisions of relativistic nuclei with the interstellar matter, then $20-40 \%$ of the energy injected in the high-energy primary CR protons is lost in the inelastic collisions before escape from the starburst region. On the other hand, Mannheim et al (2012) argued that apart from the hadronic emission from CRs the gamma-ray pulsar wind nebulae left behind by the supernovae may substantially contribute to the observed TeV luminosity of starburst galaxies. They pointed out that the hard emission detected from NGC 253 can be attributed to about $3 \times 10^{4}$ pulsar wind nebulae expected in a typical starburst galaxy.

The starburst galaxies can contribute significantly to the diffuse gamma-ray background. Recent modeling by Tamborra et al (2014) suggested that starbursts, including those with active galactic nuclei and galaxy mergers, could be the main sources of the high-energy neutrinos observed by the IceCube Observatory (see also Anchordoqui et al (2014)). Assuming a cosmic-ray spectral index of the diffuse CR population to be 2.1-2.2 for all starburst-like galaxies and extrapolating from $\mathrm{GeV}$ to $\mathrm{PeV}$ energies the authors obtained fluxes consistent with both the Fermi and IceCube data. This extrapolation assumes that there is no spectral break at $\mathrm{PeV}$ regime as it is the case in the Milky Way. To justify this one have to find a population of Pevatrons in the starburst galaxies. We shall argue in 6.3 that supernova shocks colliding with the fast winds in the compact massive star (super)clusters like Westerlund I (see 3.4 can accelerate CRs well above PeV. Since the superclusters are likely to be much more frequent in starburst galaxies CRs escaped from these accelerators may support a diffuse CR population with spectral slope harder than that observed in the Milky Way and consistent with that derived 


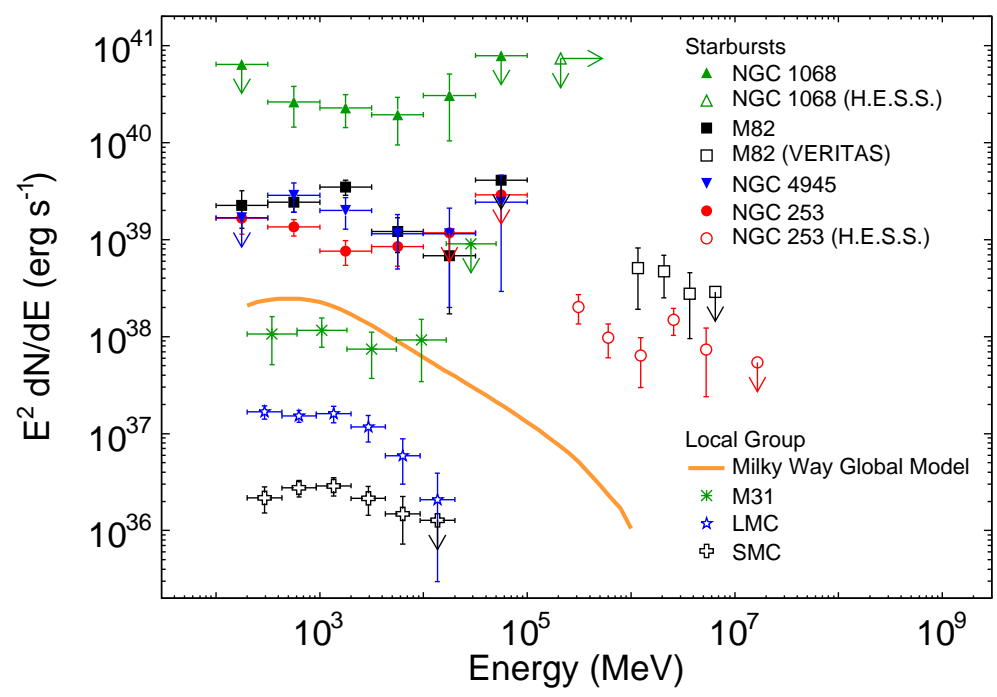

Fig. 2 The gamma-ray spectra of starburst galaxies M82, NGC 253, NGC 1068 and others detected by the Large Area Telescope aboard Fermi observatory and imaging atmospheric Cherenkov telescopes adopted from Ackermann et al (2012)

by Tamborra et al (2014). Other possible PeV neutrino sources were proposed recently including semirelativistic hypernova remnants by Liu et al (2014) and shock acceleration of $\mathrm{PeV}$ CR particles in massive galaxy mergers or collisions by Kashiyama and Mészáros (2014). In the case of massive galaxy mergers/collisions CR acceleration in colliding shock flows modeled by Bykov et al (2013b) may play a role.

\subsection{Galactic Center clusters}

Young massive star clusters of $\mathrm{M}>10^{5} \mathrm{M} \odot$ typically contain hundreds of thousands to millions of young stars within a parsec scale size core. They are called "super star clusters" (or superclusters hereafter) and are found in starburst galaxies with high SF rate (see, e.g., Portegies Zwart et al 2010). The formation of these large and dense superclusters was preferebly associated by Weidner et al (2010a) with irregular and interacting galaxies. The lack of rotational support in giant molecular clouds highly increases the probablitity of supercluster formation contrary to the picture in spiral disk galaxies like Milky Way where only a few superclusters have been found.

There is a number of impressive examples of such objects in the Local Group including the R136a cluster in Doradus 30 region of LMC, which contains as many as 39 O3 class stars - the hottest and most luminous massive stars resolved within spectroscopic observations of the Hubble Space Telescope by Massey and Hunter (1998) with the total estimated stellar mass of about $(2-3) \times 10^{4} \mathrm{M}_{\odot}$. Massey and Hunter (1998) also established that the number of high-mass stars is 
consistent with that predicted by the Salpeter-like IMF if it holds over the mass range $2.8-120 \mathrm{M}_{\odot}$. However, in a somewhat similar Galactic system NGC $3603-$ a massive star-forming $\mathrm{H}$ II region with the total mass of the associated molecular cloud about $4 \times 10^{5} \mathrm{M}_{\odot}$ where the starburst cluster of the estimated stellar mass $(1-1.6) \times 10^{4} \mathrm{M} \odot$ consists of six O3 class stars, three WNL stars, and many late O- and B-class stars - Harayama et al (2008) found that IMF can be well fitted with a power law of index $\alpha \sim 1.74$ within the mass range of 0.4 to $20 \mathrm{M}_{\odot}$. Such an IMF fit is substantially flatter then Salpeter's IMF.

Due to the extremely heavy extinction by the dust in the inner Galaxy stellar clusters are obscured in the optical band. However, radio, infrared, and X-ray observations revealed a number of young massive stellar clusters in the inner Galaxy. Within the last twenty years the Arches and the Quintuplet clusters, each of mass about $2 \times 10^{4} \mathrm{M}_{\odot}$, were discovered in the $100 \mathrm{pc}$ vicinity of the Galactic Center and thoroughly studied (see, e.g., Figer 2008, and references therein). The SF rate within the central $\sim 200$ pc of the Milky Way averaged over $\sim 10$ Gyr was estimated to be within 0.04-0.12 $\mathrm{M}_{\odot} \mathrm{yr}^{-1}$ (Crocker 2012), though local starburst events are very likely to occur in some epochs. The age of the Arches cluster is estimated as 3.5 $\pm 0.7 \mathrm{Myr}$ and the Quintuplet cluster as $4.8 \pm 1.1 \mathrm{Myr}$ (see Schneider et al 2014) thus supporting the idea of a number of local starburst events in the Galactic Center neighbourhood during the last 10 Myrs. The ionizing photon luminosity of the Arches cluster is about $4 \times 10^{51}$ photons s ${ }^{-1}$ with the total luminosity of about $6 \times 10^{7} \mathrm{~L} \odot$ and its stellar IMF appears to be of "top-heavy" type for the cluster with the estimated index $\alpha \sim 1.6 \pm 0.1$ (Figer et al 2002), which is similar to that of NGC 3603. The Arches cluster is very compact, its radius is about 0.2 pc providing a very high mass density $3 \times 10^{5} \mathrm{M}_{\odot} \mathrm{pc}^{-3}$, while the size of the Quintuplet star cluster is about a parsec.

Thermal-like and nonthermal X-ray emission was detected from both the Arches and Quintuplet star clusters with Chandra by Law and Yusef-Zadeh (2004) who found a few point like sources and sources of diffuse thermal emission near the cores of the clusters. The radius of the core of the Arches cluster is about $5^{\prime \prime}$ thus requiring the use of Chandra to resolve the point-like source contribution. A number of radio sources with X-ray counterparts were studied in both clusters with the Very Large Array. Some of the radio sources have been interpreted to be originating from the shocked colliding winds of massive binary stars Lang et al (2005). The authors interpreted three unresolved very compact sources of $<0.01$ pc size in the Quintuplet to be shocked stellar winds and estimated the total stellar wind mass injection rate for the cluster to be $\dot{\mathrm{M}} \sim 7 \times 10^{-4} \mathrm{M}_{\odot} \mathrm{yr}^{-1}$ while it is $\sim 3 \times 10^{-4} \mathrm{M} \odot \mathrm{yr}^{-1}$ for the Arches cluster. The mass-loss rates estimated from the radio data imply large kinetic power of cluster winds to be about $\gtrsim 10^{38} \mathrm{erg} \mathrm{s}^{-1}$. Such winds were considered to be the reason of the parsec scale size emission zones observed in the clusters with diffuse X-ray luminosity somewhat above $10^{34} \mathrm{erg} \mathrm{s}^{-1}$ (see e.g. Rockefeller et al 2005).

Apart from the thermal diffuse emission coming from optically thin plasma at temperatures $\sim 2 \mathrm{keV}$, non-thermal X-ray emission with relatively hard photon spectral index $\Gamma \sim 1.4$ and prominent Fe $6.4 \mathrm{keV} \mathrm{K-shell} \mathrm{line} \mathrm{emission} \mathrm{was} \mathrm{revealed}$ with a $100 \mathrm{ks}$ long Chandra observation by (Wang et al 2006) at the sub-arcminute large South-Eastern extension of the Arches cluster. The lightcurves of the Fe Kshell line emission from three bright molecular knots around the Arches cluster were found by Capelli et al (2011) to be constant over 8 years of XMM-Newton ob- 

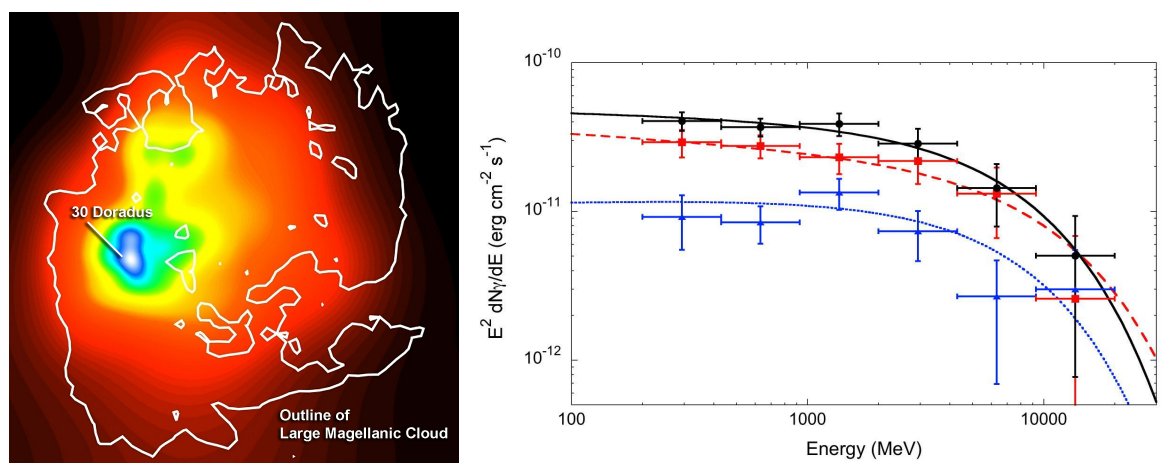

Fig. 3 Left Panel:The gamma-ray image of the Large Magellanic Cloud obtained with the Large Area Telescope aboard the Fermi Gamma-Ray Space Telescope. Bright colored region shows giant star-forming region 30 Doradus. Credit: NASA/DOE/Fermi LAT Collaboration. Right Panel: Spectrum of the gamma-ray emission from the Large Magellanic Cloud in the energy range $200 \mathrm{MeV}-20 \mathrm{GeV}$. Blue dots show the spectrum of 30 Doradus, while black and red dots show the total spectrum obtained by fitting the HII gas map and for the LMC disk, respectively. Error bars include statistical and systematic uncertainties. Adopted from Abdo et al (2010b).

servations. Tatischeff et al (2012) studied the bright $6.4 \mathrm{keV}$ Fe line features and the non-thermal emission around the Arches cluster with XMM-Newton. They found the metallicity of the ambient plasma in the cluster to be $1.7 \pm 0.2$ times the solar value and attributed the X-ray line emission to cosmic ray protons hitting the surrounding molecular gas. These authors pointed out that the Arches cluster region may be a $\mathrm{GeV}$ regime gamma-ray source potentially detectable with the Fermi Gamma-ray Space Telescope and may also produce gamma-ray line emission (see also Dogiel et al 2011a b). The model of Tatischeff et al (2012) requires large fluxes of low-energy cosmic ray ions, which can be produced if the ions are accelerated with the efficiency of a few percent at the shocks associated with the massive star cluster and some adjacent molecular cloud. Indeed such an efficiency was found plausible in a non-linear model of $\mathrm{CR}$ acceleration by shocks of velocities $\sim 1,000$ $\mathrm{km} \mathrm{s}^{-1}$ in superbubbles produced by OB stars (see Bykov 2001). Analyzing the Fe K-shell line emission from the G0.13-0.13 molecular cloud in the Galactic Center region altogether with the $74 \mathrm{MHz}$ radio continuum emission Yusef-Zadeh et al (2013) suggested that the Fe line production is due to the low-energy CR electrons interacting with the ambient matter.

The origin of the Fe K-shell line emission in the Galactic Center sources is a very interesting and still open problem. Being illuminated by powerful hard X-ray radiation from am outburst of the supermassive black hole at the Galactic Centre the neutral matter of molecular clouds can reflect and reprocess the radiation into the observed hard continuum and fluorescent $6.4 \mathrm{keV}$ line emission (Sunyaev et al 1993, Sunyaev and Churazov 1998). Indeed, Revnivtsev et al (2004) associated the hard X-ray source IGR J17475-2822 discovered with the International Gamma-Ray Astrophysics Laboratory, INTEGRAL with the giant molecular cloud Sgr B2 in the Galactic Center vicinity. They argued that the broadband spectrum of X-ray emission of Sgr B2 is due to Compton scattered and reprocessed radiation emitted by a flare of Sgr $A^{\star} 300-400$ years ago and fitted the data with a model of the central black hole, whose $2-200 \mathrm{keV}$ luminosity is about $1.5 \times 10^{39} \mathrm{erg} \mathrm{s}^{-1}$ and 
the photon spectral index is $\Gamma \approx 1.8$. Later Terrier et al (2010) analyzed $20 \mathrm{Ms}$ of INTEGRAL exposure of the Galactic Center region and found a significant weakening of the hard X-ray emission from the source in Sgr B2 associated with a molecular cloud, with characteristic time of $8.2 \pm 1.7 \mathrm{yrs}$. This fact supports the interpretation of the Sgr B2 source as an echo of a past activity of the central black hole, which came to an end only $75-155$ years ago. In the current quiet state the central black hole of mass about $4 \times 10^{6} \mathrm{M}_{\odot}$ shows luminosity less that $10^{-10}$ of its Eddington limit.

The extended characteristic $6.4 \mathrm{keV}$ line emission from low charge states of Fe being naturally produced by fluorescent scattering of the X-rays may be also excited by the low energy cosmic rays penetrating into molecular clouds in the vicinity of particle acceleration sites (see, e.g., Valinia et al 2000, Dogiel et al 2011a, Tatischeff et al 2012). Fast moving metal rich fragments of supernova ejecta may be sources of both $6.4 \mathrm{keV}$ Fe line emission as well as the high charge state Fe K-shell lines in 6.7-6.9 keV range (Bykov 2002, 2003, Bocchino et al 2012). Cataclysmic variables and coronally active binaries comprising a numerous population in the Galactic ridge are likely X-ray sources with prominent Fe lines and were proposed by Revnivtsev et al (2009) to contribute substantially to the Galactic Center X-ray emission observed by Muno et al (2009) in deep Chandra exposure.

The Arches cluster was recently observed above $10 \mathrm{keV}$ with NuSTAR (Krivonos et al 2014) in an attempt to establish the nature of the extended non-thermal Xray emission and Fe line sources. It was found that the spectrum of the hard X-ray emission extends at least up to about $40 \mathrm{keV}$ and it was not attributed to any point-like source in the Arches core, but rather shown to come from a truly extended region. The spectrum revealed by NuSTAR from a vicinity of the Arches cluster is consistent with that of thin thermal plasma of a temperature about $1.7 \mathrm{keV}$ which was previously found in the cluster core region and attributed to cluster winds thermalisation, but also contains a power-law component of photon index $\sim 1.6$. An interpretation of the hard X-ray NuSTAR observations can be suggested both within the context of the low-energy CRs interacting with the ambient cloud and within the X-ray reflection model. The X-ray reflection model is plausible if the X-ray illuminating source is associated with Sgr A* flare activity in the past, but not with the past activity of the Arches cluster itself. For the CR model the authors estimated the power of the CR proton injection above 10 $\mathrm{MeV}$ nucleon ${ }^{-1}$ in the cluster vicinity to be about $(5-8) \times 10^{38} \mathrm{erg} \mathrm{s}^{-1}$. Given the estimated above total stellar wind energy injection rate and the lack of apparent signatures of SNRs, such an injection would require some highly efficient process of kinetic power conversion into accelerated CRs. Strong ultraviolet radiation and stellar winds must have cleared and strongly heated the gas and dust in the compact clusters and thus the observational appearance of SNRs in the clusters may differ from that typical for SNRs in other phases of the ISM.

\subsection{Westerlund I supercluster and the magnetar CXOU J1647-45}

The cluster Westerlund 1 with estimated mass $\gtrsim 5 \times 10^{4} \mathrm{M}_{\odot}$ is more massive than Arches and Quintuplet, but its radius is $\sim 1 \mathrm{pc}$ resulting in the mass density $\sim 1.2 \times 10^{4} \mathrm{M} \odot \mathrm{pc}^{-3}$ which is somewhat below that in the Arches cluster (see, e.g., Figer 2008, Clark et al 2010). The cluster of the estimated age of $5.0 \pm 1.0 \mathrm{Myr}$ is 
located at a distance of about $3.8 \mathrm{kpc}$ (where $1^{\prime}$ is about $1 \mathrm{pc}$ ) in the constellation of Ara. The derived IMF slope $\alpha=1.8 \pm 0.1$ is of 'top heavy' type, i.e., it is flatter than that of Salpeter's IMF (see Lim et al 2013, and refernces therein). Muno et al (2006b) argued that for IMF with slope $1.8 \leq \alpha \leq 2.7$ for $\mathrm{M} \geq 30 \mathrm{M}_{\odot}$. the cluster should have originally contained from 80 to 150 stars of masses above $50 \mathrm{M} \odot$, which must have exploded as supernovae within the estimated age of the cluster. Then the average supernova rate over the last 1 Myr should be one in about 0.01 Myrs.

The supernovae would provide a very strong source of kinetic power of about $3 \times 10^{39} \mathrm{erg} \mathrm{s}^{-1}$ over a few Myrs time period. Observations indicated that if the scenario is appropriate only a small fraction $\lesssim 10^{-5}$ of the kinetic power estimated above is radiated in X-rays in the $5^{\prime}$ vicinity of Westerlund 1 . However, an ejecta of a supernova exploding in the cluster central region would pass through the cluster filled with tenuous hot plasma sooner than in 1,000 yrs, and thus most of the time the cluster would be free from supernova ejecta.

Diffuse X-ray emission from Westerlund 1 was revealed with Chandra observations by Muno et al (2006b) who was able to subtract the point-like X-ray source contamination from the observed emission of the cluster down to a completeness limit of $\sim 2 \times 10^{31} \mathrm{erg} \mathrm{s}^{-1}$. The rest of the $2-8 \mathrm{keV}$ emission of luminosity $L_{X}=(3 \pm 1) \times 10^{34} \mathrm{erg} \mathrm{s}^{-1}$ shows a core-halo morphology with a core of about $25^{\prime \prime}$ half-width and $\sim 5^{\prime}$ extended halo. A weak thermal plasma component of temperature $\sim 0.7 \mathrm{keV}$ was found comprising about $5 \%$ of the detected diffuse $\mathrm{X}$-ray flux while the dominant emission is due to hard continuum. The estimated emission measure $\int n_{e} n_{H} \mathrm{dV}$ is about $11_{-2}^{+4} \mathrm{~cm}^{-6} \mathrm{pc}^{3}$ within the $1^{\prime}$ inner circle and $6_{-2}^{+2} \mathrm{~cm}^{-6} \mathrm{pc}^{3}$ in the ring between $2^{\prime}$ and $3.5^{\prime}$ providing the plasma number density of $\sim 0.3 \mathrm{~cm}^{-3}$ in the outer region. Muno et al (2006b) suggested that the hard continuum component can be fitted either as hot $\gtrsim 3 \mathrm{keV}$ thin plasma with no prominent He-like Fe line emission requiring a low Fe abundance of $\sim 0.3$ of the solar value, or as power-law of photon index $\Gamma=2.7 \pm 0.2$ within the inner $1^{\prime}$ and harder index outward with $\Gamma=1.7 \pm 0.1$ in the $2.5^{\prime}-3^{\prime}$ annulus.

To address the dilemma Kavanagh et al (2011) analyzed a 48 ks XMM-Newton observation of Westerlund 1 and found He-like $\mathrm{Fe} 6.7 \mathrm{keV}$ line emission in the central $2^{\prime}$ region suggesting that the hard X-rays from the cluster core are mostly thermal in their origin. They concluded that the majority of the hard emission in the inner $2^{\prime}$ annuli likely comes from the thermalized cluster wind (see also models by Oskinova 2005) with a contribution from the pre-main sequence stellar population, while it is unlikely that SNRs are contributing significantly to the diffuse emission of Westerlund 1 at the current epoch. The Fe line signatures were not found in the outer extraction annuli by Kavanagh et al (2011) and therefore the question of the thermal or non-thermal origin of the hard emission in the outer regions has been left open.

The only clear signature of the presence of an SNR in Galactic compact clusters of young massive stars is the X-ray pulsar CXO J164710.2-455216 (CXOU J164745 afterwards) found in the Westerlund I cluster. The pulsar was discovered by Muno et al (2006a) with Chandra observations. CXOU J1647-45 has a period of $10.6107(1) \mathrm{s}$ and the estimated spin down power $\dot{\mathrm{E}} \lesssim 3 \times 10^{33} \mathrm{erg} \mathrm{s}^{-1}$ comparable to its X-ray luminosity, which imply that the pulsar is likely a magnetar powered by its high magnetic field (expected to be above $4 \times 10^{14} \mathrm{G}$ ). The pulsar is located 
at about $1^{\prime} .6$ away from the core of Westerlund 1 and possibly originates from a progenitor star of mass above $40 \mathrm{M}_{\odot}$. If a neutron star was born with a very high magnetic field (say, $\sim 10^{16} \mathrm{G}$ ) with a few ms period then the strong magnetic dipole radiation losses should slow down its period to several seconds in a few thousands years (see, e.g., Mereghetti 2008).

Note here that apart from the magnetar CXOU J1647-45 a number of other soft gamma-ray repeaters, namely SGR 052666, SGR 1900+14 and SGR 180620 are likely associated with different massive star clusters (see, e.g., Mereghetti 2008, for a review). This association may imply the origin of the magnetars from the very massive progenitor stars exploding in young massive star clusters.

Relativistic winds of newly born magnetars with initial spin rates close to the centrifugal breakup limit were suggested by Blasi et al (2000) and Arons (2003) to be the sources of ultra high energy cosmic rays. The authors pointed out that a magnetar with a magnetic dipole moment $\mu$ rotating with an angular velocity $\Omega$ has magnetospheric voltage drops across the magnetic field with magnitude $\Delta \Phi \propto \mu \Omega^{2} / c^{2} \approx 3 \times 10^{22}\left(\mu / 10^{33} \mathrm{cgs}\right)\left(\Omega / 10^{4} s^{-1}\right)^{2} \mathrm{~V}$. Arons (2003) argued for a hard power law distribution of light ions $\propto p^{-1}$ and the spectral steepening to $\propto p^{-2}$ at higher energies, with an upper cutoff energy at $10^{21}-10^{22} \mathrm{eV}$. In case of the relativistic magnetar magnetosphere the electromagnetic rate of rotational energy loss is close to that of vacuum dipole radiation $\dot{E}=c \Delta \Phi^{2}$. The electromagnetic energy emitted by the magnetar may escape the supernova envelope without substantial losses driving a relativistic blast wave in the ISM up to a parsec scale. In order to not overproduce the number of observed interstellar supershells Arons (2003) suggested that the huge initial spin-down energy of a magnetar is mostly radiated in kilohertz gravitational waves just in several hours after the birth. He also predicted that the gravitational radiation events should correlate with the bursts of UHECRs and that the Pierre Auger Observatory should see the bursts of CR particles with energy above $10^{20} \mathrm{eV}$ once in a few years. Recently, Lemoine et al (2014) proposed that CR protons of energies up to $4 \times 10^{20} \mathrm{eV}$ can be accelerated at the termination shock of the relativistic pulsar wind for a fiducial pulsar rotation period of one msec and a magnetic field of about $10^{13} \mathrm{G}$.

A few magnetars are apparently associated with known supernova remnants. Vink and Kuiper (2006) found evidences that the explosion energies of supernova remnants Kes 73 which is associated with AXP 1E 1841-045, CTB 109 (AXP 1E2259+586) and N49 (SGR 0526-66) are likely close to the canonical supernova explosion energy of $10^{51} \mathrm{erg}$, suggesting an initial spin periods of the magnetars to be about $5 \mathrm{~ms}$.

Although no radio-shell or any other standard SNR shell appearance was found yet, gamma-ray sources were detected in the vicinity of Westerlund 1 by the High Energy Stereoscopic System, H.E.S.S. in the TeV band (Abramowski et al 2012a) and by the Fermi Large Area Telescope in the GeV band (Ohm et al 2013) with gamma-ray flux $\gtrsim 10^{-11} \mathrm{erg} \mathrm{cm}^{-2} \mathrm{~s}^{-1}$. Ohm et al (2013) suggested a pulsar wind nebula is contributing to the observed gamma-ray emission and concluded that it provides a possible interpretation of the $\mathrm{GeV}$ emission, but can not explain the emission in the $\mathrm{TeV}$ band. On the other hand, they found that a model with proton acceleration in the cluster's supernova explosion(s) with subsequent interaction with the surrounding molecular material may explain both the $\mathrm{GeV}$ and $\mathrm{TeV}$ emission, but requires a very high energy input in protons and rather a slow diffusion. 


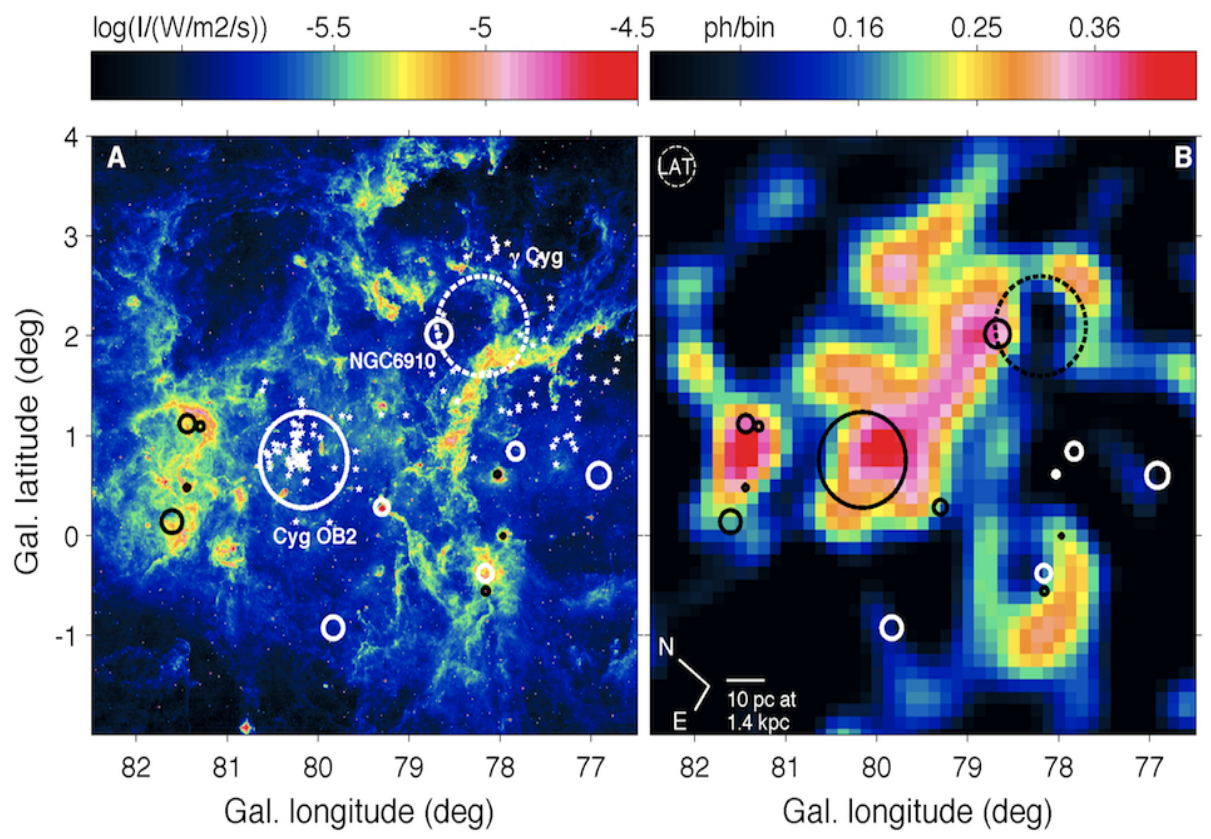

Fig. 4 Left Panel: Mid-infrared $8 \mu \mathrm{m}$ intensity map of the Cygnus X region made with Midcourse Space Experiment where rich OB stars association Cyg OB2 (white circle) and supernova remnant $\gamma$-Cygni (white dashed circle) are shown. Right Panel:Fermi-LAT 10- 100-GeV band photon count map of the Cygnus X region where a cocoon of freshly accelerated CRs of about 50-parsec-wide discovered by Ackermann et al (2011). Credit: NASA/DOE/Fermi LAT/Grenier Isabelle.

In $\$ 6.3$ we shall discuss in some detail another potentially very fast and efficient mechanism of particle acceleration in colliding shock flows suggested by Bykov et al (2013b). The colliding shock flow occurs in a compact cluster where a strong expanding supernova shock is approaching a fast wind of a nearby massive star or collides with the supersonic wind produced by the compact cluster. The colliding shock flows provide the most favourable conditions for the fast CR acceleration by the first order Fermi acceleration. In this scenario PeV regime CRs are efficiently produced on a short time scale of a few hundred years by a supernova remnant interacting with the winds in the compact cluster. Then the accelerated CRs diffuse away on a time scale of thousands years producing both gamma-ray and neutrinos by inelastic interactions of CRs with a matter of ambient clouds.

3.5 Non-thermal emission from globular clusters and superbubbles

Recently Wu et al (2014) re-analyzed Chandra observations of the old Galactic globular cluster 47 Tucanae and reported a new diffuse X-ray emission feature within the half-mass radius of the cluster. They fitted the spectrum of the diffuse emission with a unform thin thermal plasma model of $T \sim 0.2 \mathrm{keV}$ and a very hard power-law component of a photon index $\Gamma \sim 1.0$ which exponentially falls off 
outwards from the cluster core. The non-thermal X-ray luminosity of the region of interest was found to be $\gtrsim 10^{32} \mathrm{erg} \mathrm{s}^{-1}$ assuming a $4.5 \mathrm{kpc}$ distance to 47 Tucanae. The authors proposed two potential scenaria of the origin of the nonthermal emission: synchrotron/inverse Compton radiation of particles accelerated by multiple shocks of colliding winds or inverse Compton scattering of a pulsar wind. Possible signatures of non-thermal X-ray components were also reported from a nearby massive starforming region ON 2 (Oskinova et al 2010), and from a few superbubbles located in the Large Magellanic Cloud (see e.g. Bamba et al 2004, Maddox et al 2009).

Detailed study of 30 Dor C superbubble with XMM-Newton was performed recently by Kavanagh et al (2014). Apart from the hard X-ray shell which is highly correlated with the $\mathrm{H}_{\alpha}$ and radio shells the non-thermal X-ray emission was detected from all regions of 30 Dor $\mathrm{C}$ as well. The authors found that the non-thermal $\mathrm{X}$-ray emission may be fitted with a power-law model but an exponential cut-off synchrotron model may fit the spectrum better. The maximal energy of electrons $\epsilon_{\max }$ which is required to justify the X-ray synchrotron model was estimated by Kavanagh et al $(2014)$ as $\epsilon_{\max } \approx 80 \mathrm{TeV}(B / 10 \mu G)^{-1 / 2}$. The synchrotron losses time of the electrons is below 1.5 kyrs and therefore efficient particle acceleration is required. Kavanagh et al (2014) argued that 30 Dor C is currently undergoing a phase of high energy particle acceleration by massive star winds and perhaps supernova remnants expanding in the hot superbubble. The maximal energy of the electrons might be somewhat lower than that derived by Kavanagh et al (2014) if one account for the effect of turbulent magnetic fields in the superbubble on the spectra of the synchrotron radiation .

The spectrum of the giant starforming region 30 Doradus in the LMC in the energy range from $100 \mathrm{MeV}$ to $100 \mathrm{GeV}$ obtained with the Fermi LAT is shown in Fig. 3 (the blue data points). The spectrum of the source shown in the right panel is consistent with a photon index $\Gamma \approx 2$ and the estimated gamma-ray luminosity is $\gtrsim 4 \times 10^{36} \mathrm{erg} \mathrm{s}^{-1}$ (see, e.g., Abdo et al 2010b). The luminosity is well below the gamma-ray luminosities of $10^{39}$ to $10^{41} \mathrm{erg} \mathrm{s}^{-1}$, which are implied from recent observations of the powerful starburst galaxies M82, NGC 253, NGC 1068, NGC 4945 (see, e.g., Abdo et al 2010a, Ackermann et al 2012) (they are indicated in Fig. 2), but it is substantially larger than the 1-100 GeV luminosity of the Cygnus cocoon discovered by (Ackermann et al 2011) as discussed below.

The Fermi LAT telescope discovered a cocoon of about 50 parsec width located in between Cygnus OB2 and $\gamma$-Cygni SNR with a peak of the emission close to the massive-star cluster NGC 6910 (Ackermann et al 2011). The cocoon in Figure 4 shows signs of enhanced fluxes of low energy cosmic rays likely powered by multiple shocks of fast stellar winds and SNRs located in the Cyg OB2 region, which overlaps with a TeV source MGRO J2031+41. Pulsar wind nebulae expected in the region with multiple SNRs could also contribute to these fluxes, but Ackermann et al (2011) pointed out that the young pulsars J2021+4026 and J2032+4127 are unlikely to explain the Cygnus cocoon emission. The gamma-ray luminosity of the cocoon in the $1-100 \mathrm{GeV}$ range is about $(9 \pm 2) \times 10^{34} \mathrm{erg} \mathrm{s}^{-1}$ assuming the 1.4 $\mathrm{kpc}$ distance to the Cygnus OB2 region. Recently ARGO-YBJ reported an identification of the extended TeV source ARGO J2031+4157 ( MGRO J2031+41) with the Fermi Cygnus cocoon. They fitted 0.2-10 TeV spectrum of ARGO J2031+4157 with a photon index $\Gamma=2.6 \pm 0.3$ The ARGO-YBJ Collaboration et al (2014) while Fermi LAT spectrum below $100 \mathrm{GeV}$ seems to be better fitted with a flat- 

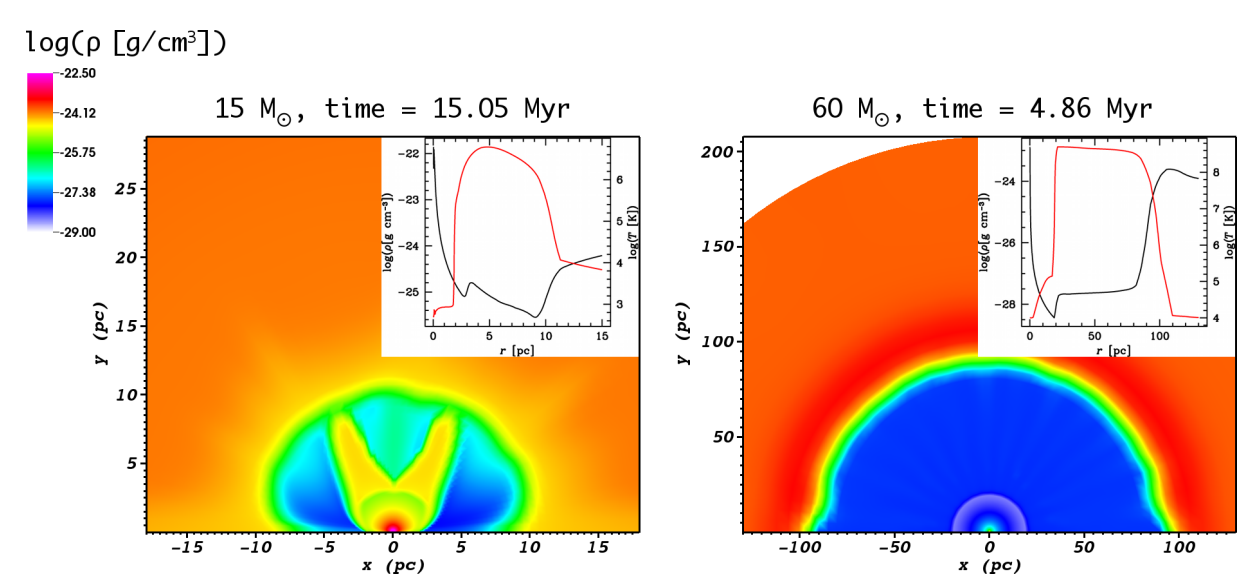

Fig. 5 Typical pre-SN aspect of the circumstellar matter around a $15 \mathrm{M}_{\odot}$ star (left panel) ending its stellar life as a red supergiant (and exploding as a type IIP $\mathrm{SN}$ ), and around a $60 \mathrm{M}_{\odot}$ star (right panel), ending as a Wolf-Rayet star (exploding as a type Ibc SN). In the small windows the mean density (black solid line) and temperature (red solid line) are shown as a function of the radius. Adopted from Georgy et al 2014).

ter power-law photon index $\sim 2.2$. All of these systems of different spatial scales and injected kinetic power are characterized by spectra of photon indexes 2-2.5 in $\mathrm{GeV}-\mathrm{TeV}$ regimes studied with Fermi LAT and ground-based TeV telescopes.

Gamma-ray observations in the $\mathrm{TeV}$ regime with ground based atmospheric Cherenkov telescopes H.E.S.S., MAGIC, VERITAS, and Milagro, a water Cherenkov detector reaching a high sensitivity, revealed a number of sources in the Cygnus region (see, e.g., Aharonian et al 2002, 2006, Albert et al 2008, Ong and for the VERITAS Collaboration $\mid 2013$, H.E.S.S. Collaboration et al |2011) and of young massive star clusters (see, e.g., Abramowski et al $2012 \mathrm{a} \mid$ H.E.S.S. Collaboration et al 2011). Some of the sources are likely extended. While the exact identification of the extended sources is still to be made with the multiwavelength observations, the most plausible associations are related to either pulsar wind nebulae or multiple shock systems within OB star clusters and SNRs (see, e.g., Butt et al 2003 , Torres and Reimer 2013, and references therein).

\section{Hydrodynamical models of massive star winds and superbubbles}

To interpret adequately the multi-wavelength observational data on the starforming and starburst regions one has to rely on models. The evolution of an SF system is an integral part of the ISM ecology and it starts from molecular cloud formation and ends with the destruction of the cloud by radiation and winds of massive stars, supernova explosions, dispersion of open clusters of massive stars or massive globular cluster formation. Above we have briefly discussed some aspects of the first stages of the SF process and a more detailed discussion can be found in Bromm and Larson (2004), McKee and Ostriker (2007), Zinnecker and Yorke (2007), Elmegreen (2011a), Kennicutt and Evans (2012), Sanchez Almeida et al (2014), Krumholz (2014), Molinari et al (2014), and Tan et al (2014). 
Basic physics of the mechanical and thermal equilibrium of rotating massive stars and their evolution has been thoroughly reviewed by Maeder and Meynet (2012) with implications to population synthesis models (see, e.g., Georgy et al 2014). Radiative forces of high luminosity young massive stars are accelerating near-surface layers initiating fast winds and stellar mass loss. Simulations of the line-driven stellar winds require a careful study of the instabilities at different scales, clumping and porosity effects Abbott (1982), Owocki et al (1988), Kudritzki and Puls (2000), Puls et al (2008), Owocki (2014) to model the dependence of mass loss and wind terminal velocity on the metallicity and stellar rotation and to establish the maximal mass limit and pursue evolution of young massive stars.

The mass loss rates of young massive stars are typically within the range $10^{-7}<\dot{\mathrm{M}}<10^{-4} \mathrm{M}_{\odot} \mathrm{yr}^{-1}$ (and may be as high as $5 \times 10^{-4} \mathrm{M}_{\odot} \mathrm{yr}^{-1}$ for a relatively short luminous blue variable phase) with the terminal velocities reaching $3,000 \mathrm{~km} \mathrm{~s}^{-1}$. For example, the total kinetic energy input from a wind of a $60 \mathrm{M}_{\odot}$ star is about $8 \times 10^{50}$ ergs for a mean terminal velocity of $2,000 \mathrm{~km} \mathrm{~s}^{-1}$ before the supernova explosion. The models of stellar evolution by Chiosi and Maeder (1986), Maeder and Meynet (1988), Lamers and Cassinelli (1999) predict that such a star would eject into the interstellar medium about $29 \mathrm{M}_{\odot}$ of hydrogen, $\sim 8$ $\mathrm{M}_{\odot}$ of $\mathrm{He}$, and $\sim 1 \mathrm{M}_{\odot}$ of $\mathrm{C}$ and $\mathrm{O}$ (almost $38 \mathrm{M}_{\odot}$ of gas in total). A very useful recipe to estimate the mass-loss rate as a function of the stellar mass and luminosity, effective temperature and terminal velocity of the wind for different metal abundances can be found in Vink et al (2000, 2001). Magnetic fields in OBstars are thought to be at the level of $\sim 100 \mathrm{G}$. They play a role in the structure and evolution of young massive stars maintaining a rotation law to be close to uniformity (see, e.g., Maeder et al 2009). The magnetic coupling is essential to regulate the interconnection between the differential rotation (which it tends to suppress) and the meridional circulation. The magnetic braking effect reduces stellar rotation if the star's mass loss is strong.

The origin of magnetic fields of different scales in massive stars is now a subject of discussions. The dynamo-type models are being considered along with a stellar merging process where the binary evolution may result in a rapid mass transfer phase accompanied by magnetic field amplification. This may account for magnetic fields of about $10 \%$ of O-type stars which are thought to be produced via merging of two main sequence stars (see e.g. Langer 2013).

Massive early type star winds inject energy and momentum into the surrounding medium to blow bubbles of different scale sizes surrounded by shells (see, e.g., Castor et al 1975, Weaver et al 1977, Lamers and Cassinelli 1999, Georgy et al 2013). Below we will briefly review some recent models of bubbles formed by individual massive early type stars as well as superbubbles produced by collective actions of clustered massive stars and supernovae on the time scale of $\gtrsim 10$ Myrs and compare them with available observational data depicted by $\mathrm{Chu}(2008)$ who discuss OB associations, hot interiors of superbubbles, swept-up dense shells, and interfaces between dense shells (see also Feigelson et al 2013).

In Figure 5 wind structure is illustrated along with the recent results of twodimensional axisymmetric hydrodynamical simulations of the circumstellar medium around $15 \mathrm{M}_{\odot}$ and $60 \mathrm{M}_{\odot}$ stars at rest taken at the pre-supernova stages perfomed by Georgy et al (2013). The simulations were made for a grid of massive stellar models from 15 to $120 \mathrm{M}_{\odot}$ the stellar evolution was followed from the zero-age main-sequence stage to the stage of supernova explosion. Gas velocity, 


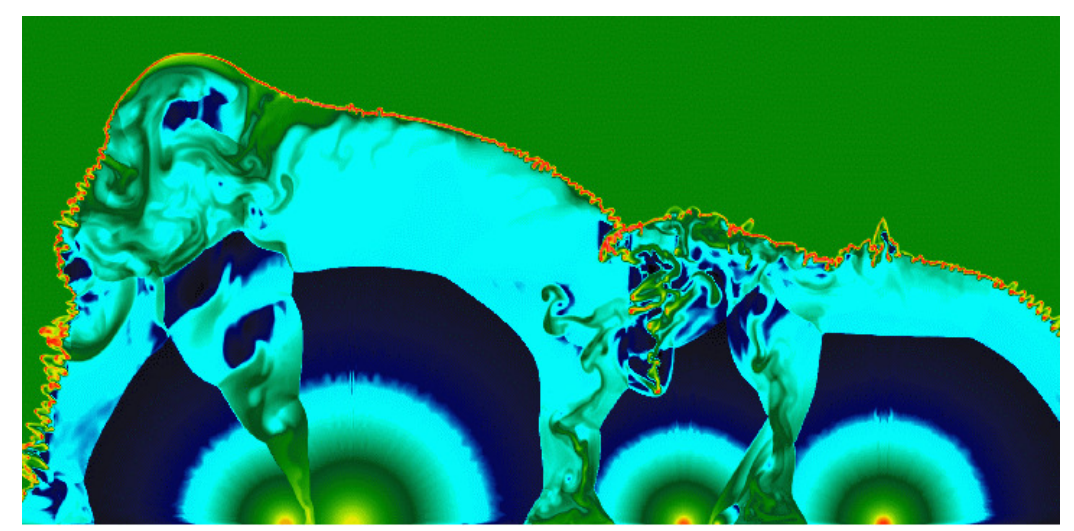

Fig. 6 Hydrodynamical simulation of an axisymmetric mini-star cluster of 5 stars (from Georgy et al (2014)). Thin shell instabilities develop in regions of interactions of different winds and of wind-interstellar medium. Gas densities are shown in color: $10^{3} \mathrm{~cm}^{-3}$ (white), $10 \mathrm{~cm}^{-3}$ (red), $1 \mathrm{~cm}^{-3}$ (green), $10^{-2} \mathrm{~cm}^{-3}$ (blue).

density and temperature profiles are shown in the insets (for details see Georgy et al (2013)). Fast wind of velocity of a few thousands $\mathrm{km} \mathrm{s}^{-1}$ is confined in a region of a few pc size and terminated at a strong shock where the tenuous gas is heated up to temperatures $10^{6}-10^{8} \mathrm{~K}$ and the hot bubble may extend for tens of parsecs. The bubble in the simulation expands slower than it is predicted by the self-similar model and its size depends on the metallicity of the central star. The low-metallicity stars have relatively smaller bubbles because their winds are weaker. The effect of the stellar motion on the structure of the circumstellar medium may be important and was studied by Mackey et al (2014) who have calculated a grid of models of bow shocks around main sequence and evolved massive runaway stars.

Since the young massive stars are highly clumped in the region where they originate, and the mean distances between the stars are typically smaller than the sizes of individual star bubbles, the structure of the circumstellar medium in such a region would be more complex. Velázquez et al (2003) performed a numerical hydrodynamic simulation of the interaction of supernova ejecta with a stellar wind. They modeled the X-ray emission from a supernova remnant — stellar wind collision region and found shell-like structures of enhanced X-ray emission. It is instructive to note that the simulated system at some evolutional stage (of about some thousands of years duration) may become a very efficient accelerator of energetic particles. It may transfer about a half of the kinetic ram pressure into the energetic non-thermal particles as demonstrated within the one-dimensional non-linear kinetic model of Bykov et al (2013b).

A 2D hydrodynamical model of the temporal evolution of an aspherical circumstellar bubble created by two massive stars of $25 \mathrm{M}_{\odot}$ and $40 \mathrm{M}_{\odot}$ separated by 16 pc in a cold $20 \mathrm{~cm}^{-3}$ medium, which included the stage of supernova explosion was presented by van Marle et al (2012). In Figure 6 adopted from Georgy et al (2013) we illustrate density distribution at some intermediate stage of a simulated $2 \mathrm{D}$ evolution of a cluster of 5 massive stars. 
Evolution and X-ray spectra of three massive star system of 25, 32, and $60 \mathrm{M} \odot$ in a background matrix of $10 \mathrm{~cm}^{-3}$ density was studied by Krause et al 2013 2014) in 3D hydrodynamics with an account for optically thin radiative cooling and photo-electric gas heating. They found that the energy injected by supernovae is entirely dissipated in a superbubble on the timescale of about $1 \mathrm{Myr}$, and that the deposition from the stars to the superbubble did not vary substantially if the massive stars were localized within a distance of $\lesssim 30 \mathrm{pc}$. The simulations showed the outer dense shell thickness to be $\sim 0.1$ of the superbubble size in accordance with observations. The X-ray luminosity of a superbubble increases by a factor $\lesssim 100$ for a period of about $0.1 \mathrm{Myr}$ after a supernova explosion reaching a peak luminosity $\sim 10^{36} \mathrm{erg} \mathrm{s}^{-1}$. Rogers and Pittard (2013, 2014) simulated distraction of a giant molecular cloud clump of about $3240 \mathrm{M}_{\odot}$ mass by three O-stars localised within a 4 pc radius.

The simulations demonstrated a highly structured environment into which the supernova energy is released after 4.4 Myrs of the system evolution, allowing most of the supernova energy to reach wider environments. The molecular component is completely destroyed and dispersed in $6 \mathrm{Myr}$. The authors found that the $\mathrm{X}$ ray luminosity of the system is about $10^{34} \mathrm{erg} \mathrm{s}^{-1}$ at the early stages of the evolution and during short periods of Wolf-Rayet stages. However, it goes down to about $1.7 \times 10^{32} \mathrm{erg} \mathrm{s}^{-1}$ between $1-4$ Myrs and it can increase to $\sim 10^{37} \mathrm{erg} \mathrm{s}^{-1}$ for relatively short time periods $\gtrsim 4,600$ yrs after each supernova explosion. Because of the leakage of hot gas material through gaps in the outer shell, the X-ray luminosities obtained in 3D simulations by Rogers and Pittard $(2014)$ are generally considerably fainter than those predicted within spherically-symmetric bubble models.

Mac Low and McCray (1988) presented a general view on the ISM in the vicinities of starforming regions where correlated supernova explosions in OB associations create a superbubble: a large, thin, shell of cold gas surrounding a hot pressurized interior. The dynamics of a superbubble was approximated as that of a source of continuous mechanical luminosity assuming that supernova blast waves become subsonic before they reach the walls of the shell. A thin shell (the Kompaneets' approximation) is widely used (see, e.g., Bisnovatyi-Kogan and Silich 1995) to describe supershell propagation in inhomogeneous interstellar medium. Superbubbles may be blown out of the H I layer into the halo. The evolution of superbubbles in an exponential ISM density distribution is a subject of recent analytic and numerical studies (see, e.g., Baumgartner and Breitschwerdt 2013. Booth et al 2013). In this context Dorfi and Breitschwerdt (2012) suggested a model which can explain the power-law distribution of CRs between the "knee" and the "ankle". The impact of supernovae in superbubbles on the galactic ecosystem was discussed in various aspects by Hennebelle and Iffrig (2014) and by Wang (2014). One should have in mind the existence of a feedback effect of supernova and superbubbles on the global galactic ecology. This is the effect of large scale galactic magnetic field amplification by the cosmic-ray-driven dynamo on the timescale of about 150 Myr simulated by Hanasz et al (2009), Siejkowski et al (2014). Price and Bate (2008) demonstrated that even a very low magnetic field is able to significantly influence the star formation process. Global properties of superbubbles can be affected seriously by the local effects of magnetic field, thermal conduction, turbulent mixing as well as by energy exchange with accelerated particles which are connected with the thermal plasma motions via fluctuating magnetic 
fields produced by CR-driven instabilities (see, e.g., Bell 2004, Zweibel and Everett 2010, Bykov et al 2012, Schure et al 2012, Blasi 2013) and may comprise a sizeable fraction of the free energy of a superbubble (Bykov 2001, Butt and Bykov 2008).

\section{Observational appearance of massive star winds and superbubbles: the non-thermal emission}

The structure of the circumstellar medium modified by a strong wind of a massive star and a presence of molecular clouds seriously influence both the thermal and the non-thermal appearance of a supernova remnant (see e.g. Chevalier 1999, 2005, 2014, Bykov et al 2000, Ellison et al 2012). The structure of magnetic fields in the winds of massive stars was discussed by, e.g., Walder et al (2012). The magnitude of magnetic field in the close vicinity of the progenitor star may be high enough to allow acceleration of particles in very young supernova remnants to energies well above the PeV regime (e.g., Voelk and Biermann 1988, Ptuskin et al 2010). On the other hand, magnetic field in the outer regions of the wind of a slowly rotating massive star may drop below a $\mu \mathrm{G}$ level. The low magnetic field allows the inverse Compton interpretation of the observed GeV-TeV gamma-ray photons from synchrotron X-rays dominated supernova shell RX J1713.7-3946 in the frame of the diffusive shock acceleration model with strong magnetic field amplification. In this model the low magnetic field in the outer region of pre-supernova wind is amplified by $\mathrm{CR}$ driven instabilities by a factor of $\sim 50$ (say, from $0.4 \mu \mathrm{G}$ to $20 \mu \mathrm{G})$ (Ellison et al 2012). The gamma-ray image of this SNR morphologically resembles the synchrotron X-ray map (Aharonian et al 2007). The models, which explain the origin of the gamma-ray photons via the inverse Compton scattering of background photons by the synchrotron X-ray emitting electrons typically require magnetic field in the emission region to be $\sim 20 \mu \mathrm{G}$ (see, e.g., Vink 2012, for a discussion). This is difficult to reconcile with an efficient particle acceleration at supernova shock, which results in a high magnetic field amplification factor (above 10 ) if the shock is propagating into the standard ISM with magnetic field of a few $\mu \mathrm{G}$ observed under the typical ISM conditions (Beck 2012, Heiles and Haverkorn 2012 ), unless the SNR is expanding into the wind of a pre-supernova.

\subsection{Colliding wind binaries}

The wind collision in early-type binary systems is the next level of complexity. The wind structure, position and shape of the contact discontinuity between the stars, double-shock structures, as well as the post-shock flows were examined by Stevens et al (1992) and later by Parkin and Pittard (2008), who correspondingly used 2D and 3D hydrodynamic codes with an account for radiative cooling and some instabilities. The models were aimed to describe the symbiotic systems and gamma-ray binaries, and also binary systems with O-type and Wolf-Rayet stars including the enigmatic object in $\eta$-Carinae. The colliding wind binaries were long known to be sources of non-thermal radio emission Eichler and Usov (1993), Dougherty et al (2005), Pittard and Dougherty (2006), De Becker (2007) and the catalog of colliding wind binaries with non-thermal particle acceleration compiled by De Becker and Raucq (2013) contains 43 objects. 
Dougherty et al (2005) presented milliarcsecond resolution Very Long Baseline Array observations of the colliding-wind binary WR 140 (HD 193793) at different orbital phases. WR 140 is a massive star binary with estimated masses of $20 \pm 4$ $\mathrm{M}_{\odot}$ for the $\mathrm{W}-\mathrm{R}$ star and $54 \pm 10 \mathrm{M}_{\odot}$ for the $\mathrm{O}$ star and a period of 7.9 days. The system has a highly elliptical orbit where the stellar separation varies between $2 \mathrm{AU}$ at periastron and about $30 \mathrm{AU}$ at apastron. The mass loss rate determined from the thermal free-free emission of WR wind is $\gtrsim 2 \times 10^{-5} \mathrm{M}_{\odot} \mathrm{yr}^{-1}$ being a subject of uncertainty due to likely clumping of the wind of a terminal velocity $\sim 3,000$ $\mathrm{km} \mathrm{s}^{-1}$. The estimated total kinetic luminosity of WR 140 is $\sim 6 \times 10^{37} \mathrm{erg} \mathrm{s}^{-1}$ while the radio luminosity is $\sim 2.6 \times 10^{30} \mathrm{erg} \mathrm{s}^{-1}$ (see De Becker and Raucq 2013). The total radio flux measured with VLBA does not change from one orbit to the next, but shows strong variations with the orbital motion exhibiting both optically thin and optically thick synchrotron radio emission (Dougherty et al 2005). Pittard and Dougherty (2006) estimated the kinetic power in the wind - wind collision to be $\sim 510^{36} \mathrm{erg} \mathrm{s}^{-1}$ of which - they suggested - about $0.5 \%$ is being transferred to the broad band non-thermal emission observed from radio to gamma-rays, which is due to energetic particles accelerated by shocks (c.f. Eichler and Usov 1993. Bednarek 2005) or magnetic field reconnection. By now Fermi LAT observations provided an upper limit of $\sim 9.6 \times 10^{-9} \mathrm{~cm}^{-2} \mathrm{~s}^{-1}$ (for one energy bin between 95.6 MeV and 44.9 GeV) for the gamma-ray emission from WR 140 (Werner et al 2013). The same authors established upper limits on gamma-ray emission from a number of other colliding wind binaries.

Gamma-ray emission and hard X-ray non-thermal emission was detected from $\eta$-Carinae (Tavani et al 2009, Abdo et al 2009, Farnier et al 2011, Dubus 2013) which is considered as a likely case of colliding wind binary. No clear evidence for a non-thermal synchrotron component in the observed radio emission of $\eta$-Carinae has been reported yet, possibly due to a high free-free absorption (see, e.g., De Becker and Raucq 2013, and references therein). An analysis of broad-band X-ray, optical and near-infrared observations carried out within about 60 yrs (Damineli et al 2008) revealed the binarity of the system with the period of $2022.7 \pm 1.3$ days (about $5.54 \mathrm{yrs}$ ). The total mass of the system is likely $\gtrsim 110 \mathrm{M} \odot$. The primary star $\eta$-Carinae A is likely a luminous blue variable star which is characterized by a great mass loss rate estimated as $2.5 \times 10^{-4}-10^{-3} \mathrm{M}_{\odot} \mathrm{yr}^{-1}$ and the wind terminal velocity $\lesssim 600 \mathrm{~km} \mathrm{~s}^{-1}$ with giant eruption events in the past that may have ejected some material at velocities about $3,000 \mathrm{~km} \mathrm{~s}^{-1}$. The probable companion star named $\eta$-Carinae B has not been directly observed, but the analysis of X-ray observations hinted on the presence of its wind of high terminal velocity $\gtrsim 3,000$ $\mathrm{km} \mathrm{s}^{-1}$ and the mass loss rate $\sim 10^{-5} \mathrm{M}_{\odot} \mathrm{yr}^{-1}$ (Pittard and Corcoran 2002, Groh et al 2010). The system is located at the distance of $2.3 \mathrm{kpc}$ and has a complex geometry of winds and shells (see, e.g., Madura et al 2013).

Analyzing Fermi LAT data on the source FGL J1045.0-5942 associated with $\eta$-Carinae together with INTEGRAL IBIS observations Farnier et al (2011) suggested a two-component gamma-ray spectrum of the source with a hard component of photon index $\Gamma=1.69 \pm 0.12$ with exponential cut-off at $\sim 1.8 \pm 0.5$ $\mathrm{GeV}$ present in the energy range $0.2-8 \mathrm{GeV}$. The authors suggested the leptonic origin of the component from the inverse Compton upscattering of the background photons. The second component is a power-law of index $\Gamma=1.85 \pm 0.25$ in the high-energy regime $10-100 \mathrm{GeV}$ with the normalization flux lower than that of 
the hard one; it was attributed to the hadronic component due to pion decays. The total gamma-ray luminosity of FGL J1045.0-5942 is about $10^{34} \mathrm{erg} \mathrm{s}^{-1}$.

\section{Particle acceleration and non-thermal radiation in starburst complexes}

Non-thermal emission detected in the broad band spectra of colliding wind binaries, supernova remnants, young massive star clusters, superbubbles and starburst galaxies are the signatures of violent energy release in the systems and of particle acceleration. Intense plasma motions powered by gravitation, anisotropic radiation flows, and magnetic fields may transfer a part of the available free energy to a population of highly non-thermal particles. The efficiency of the energy conversion depends on the particular acceleration mechanism and may reach tens of percent. Two kinds of particle acceleration processes are evidently at work in different astrophysical objects: those related to magnetic field reconnections and to magnetohydrodynamic shocks and turbulence.

To release the free energy of highly magnetized plasma systems with globally frozen-in magnetic fields effective dissipative processes have to work in a regime with infrequent Coulomb collisions as well. Electric fields associated with the magnetic field reconnection/dissipation regions which allow to change the field topology accelerate charged particles and heat plasma.

The second type of process is most likely operating in starburst complexes given that the radiative pressure driven winds of massive stars are not highly magnetized and that fast MHD shocks are the most common phenomena in supersonic flows of colliding winds and supernovae. Before a discussion on particle acceleration by shocks and turbulence we will briefly summarize some facts concerning particle acceleration during magnetic reconnection.

\subsection{Particle acceleration due to magnetic field reconnection}

Efficient particle acceleration may occur in the systems where the magnetic field configuration storages free energy, for example in stellar atmospheres, magnetotails of fast moving stars, highly magnetized winds or jets. Rapid reconnection of magnetic fields of different topologies may happen in spontaneous current sheets or be due to an external driving force (see, e.g., Zweibel and Yamada 2009). Mechanisms of direct acceleration of charged particles by the reconnecting magnetic field were studied in detail both analytically and numerically (see, e.g., Zelenyi et al 1990, Hoshino 2005, Birn and Priest 2007, Drake et al| 2006, Pritchett||2006, Lazarian and Opher 2009, Artemyev et al 2013, Birn et al 2012).

An early analytical study of test particle orbits around X-line field topology with and without a guide field (where the electrons remain magnetized) was performed by Bulanov and Sasorov (1976) and later advanced with Particle-in-Cell (PIC) technique (see, e.g., Oka et al 2010, Bessho and Bhattacharjee 2012, Sironi and Spitkovsky 2012). Bulanov and Sasorov (1976) came up with an exponential asymptotic shape of the energetic electron spectra in both relativistic and nonrelativistic regime. In the relativistic regime $N(\gamma) \propto \exp \left(-\left(\gamma / \gamma_{m}\right)^{1 / 2}\right)$ where the electron Lorentz factor $\gamma \gg 1$ and the maximal Lorentz factor $\gamma_{m}$ can be roughly 
estimated as $\gamma_{m} \sim \delta_{\mathrm{s}} \times \epsilon^{2}$. Here $\epsilon$ is the ratio of the electric field inside the X-line to the amplitude of magnetic field at the boundary of the current sheet system, whose geometry is characterized by the dimensionless current sheet stretching $\delta_{\mathrm{s}}$. Under typical current sheet conditions $\epsilon \sim 10^{-3}-10^{0}$, while $\delta_{\mathrm{s}} \sim 10^{4}-10^{6}$ for the electrons and $\delta_{\mathrm{s}} \sim 10^{3}-10^{4}$ for the ions. Recent two-dimensional PIC simulations of magnetic reconnection and particle acceleration in relativistic Harris sheets in low-density electron-positron plasmas with no guide field performed by Bessho and Bhattacharjee (2012) revealed particle spectrum $N(\gamma) \propto \gamma^{-1 / 4} \exp \left(-\left(\gamma / \gamma_{m}\right)^{1 / 2}\right)$.

Due to spatial and temporal localization of a magnetic reconnection region the efficiency of charged particle acceleration is substantially limited. In particular, formation of exponential tails of accelerated particle distributions results from the instability of charged particle motion in the vicinity of magnetic field null points. The amounts of accelerated particles and their energies can be significantly increased in case the of multiple reconnection when one considers formation of several magnetic islands interacting with each other (Hoshino 2012). Transient magnetic reconnection in hot plasma is responsible for generation of strong plasma jets, which accelerate electrons via adiabatic mechanisms (Birn et al 2004, AshourAbdalla et al 2011). Hall electric fields play an importnat role in electron trapping and acceleration in the vicinity of a reconnection site (Hoshino 2005, Zharkova et al 2011).

Additional effects responsible for charged particle acceleration within a reconnection region are due to resonant and nonresonant charged particle interaction with electromagnetic turbulence generated by plasma flows originated from the reconnection site (e.g., Grigis and Benz 2006, Petrosian and Bykov 2008, Bykov and Fleishman 2009). Strictly speaking, only models including turbulence or multiple reconnections pretend to reproduce the power law energy distribution of accelerated particles. In these models piece-wise power law distributions of accelerated particles may have hard spectral indexes due to fast acceleration rates and extended acceleration regions. The essential part of such models corresponds to stochastic charged particle interaction with magnetic field structures representing magnetic islands, current filaments and strong waves.

It was recently established that magnetic field reconnection effects are likely responsible (under some specific conditions) for dissipation of fully developed magnetic turbulence. In the scenario considered by Servidio et al (2011) spontaneous magnetic reconnection is locally driven by the dynamical forces under boundary conditions provided by the turbulence.

With kinetic simulations that span a wide dynamical range from the macroscopic fluid scales down to the electron scales Karimabadi et al (2013) demonstrated that a turbulent cascade results in formation of coherent structures of multiple current sheets that steepen to the electron scales. This results in strong localized heating of the plasma due to magnetic reconnection and the dominant heating mechanism is related to parallel electric fields within the current sheets leading to anisotropic distributions of electrons and ions. Plasma heating by current sheets was found to be locally several orders of magnitude more efficient than wave damping. This mechanism may be very important to understand plasma heating due to dissipation of strong collisionless magnetic turbulence in fast winds of massive stars, colliding binary systems, supernova shells and superbubbles. By now dissipation of collisionless turbulence has been treated within MHD models at best by introducing some ad hoc phenomenological macroscopic viscosities and 
an important issue of the electron to ion heating rate ratio shall be addressed with the new approaches to come.

\subsection{Particle acceleration by collisionless shocks}

Shock waves are thought to be the main gas heating and particle acceleration agents in astrophysical flows. We shall discuss below some of the properties of collisionless shocks with an accent on the production of non-thermal components in the form of energetic particles, fluctuating magnetic fields and radiation, which are rather common because of the very slow rate of the Coulomb relaxation process in this type of flows. The large number of degrees of freedom in the astrophysical collisonless shocks determines their multi-scale nature.

It was established more than fifty years ago both observationally and theoretically that collisionless shocks do exist in the mildly magnetized plasmas (see, e.g., Sagdeev 1966, Tidman and Krall 1971). Nevertheless, a non-trivial question still remains - whether the collisionless shocks may occur in a hot unmagnetized plasma, as well as in the highly magnetized plasmas. Two-dimensional PIC simulations of the structure of nonrelativistic collisionless shocks in unmagnetized electron-ion plasmas by Kato and Takabe (2008, 2010) revealed that the energy density of the magnetic field generated by a Weibel-type instability within the shock transition region typically reaches $1 \%-2 \%$ of the upstream bulk kinetic energy density. The width of the shock transition region was found to be about 100 ion inertial lengths, independent of the shock velocity. The hybrid plasma simulations with kinetic treatment of ions and fluid electron description (see, e.g., Winske and Quest 1988, Winske et al 1990, Giacalone et al 1997, Treumann 2009, Gargaté and Spitkovsky 2012, Burgess and Scholer 2013) allow to study domains of some thousands gyroradii of upstream protons around non-relativistic shocks. Two-dimensional hybrid simulations of a quasi-parallel shock with the Alfven Mach number $M_{a}=6$ by Gargaté and Spitkovsky (2012) revealed a power law distribution of energetic ions of index about $2 \pm 0.2$ in the shock downstream. The modelled energetic particle population contained about $15 \%$ of the upstream flow energy. Recent 3D hybrid simulations of non-relativistic collisionless strong shocks with $M_{a}=6$ in the domain of $2000 \times 200 \times 200$ ion inertial lengths performed by Caprioli and Spitkovsky (2013) showed magnetic field amplification (MFA) in the shock upstream and particle acceleration effects.

A distinctive feature of collisionless shocks in extended astrophysical flows is their ability to transfer a sizeable fraction of mechanical power of the flow to non-thermal particles and fluctuating magnetic fields by means of the first order Fermi acceleration mechanism called the diffusive shock acceleration (see, e.g., Bell 1978, Axford 1981, Drury 1983, Blandford and Eichler 1987, Berezhko and Krymskii 1988, Jones and Ellison 1991, Malkov and Drury 2001, Bykov et al 2008,

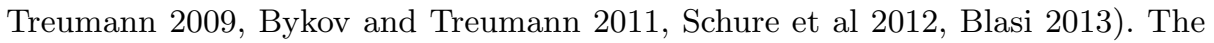
high efficiency of particle acceleration which may be well above $10 \%$ as deduced, in particular, from observations of young supernova remnants (see, e.g., Vink 2012 , Helder et al 2012, Blasi 2013) implies strong coupling between the accelerated particle population and the shock structure. The coupling is realized through the electromagnetic fluctuations carried with the shock flow and responsible for inelastic scattering the particles. The fluctuations are strongly amplified by CR driven 

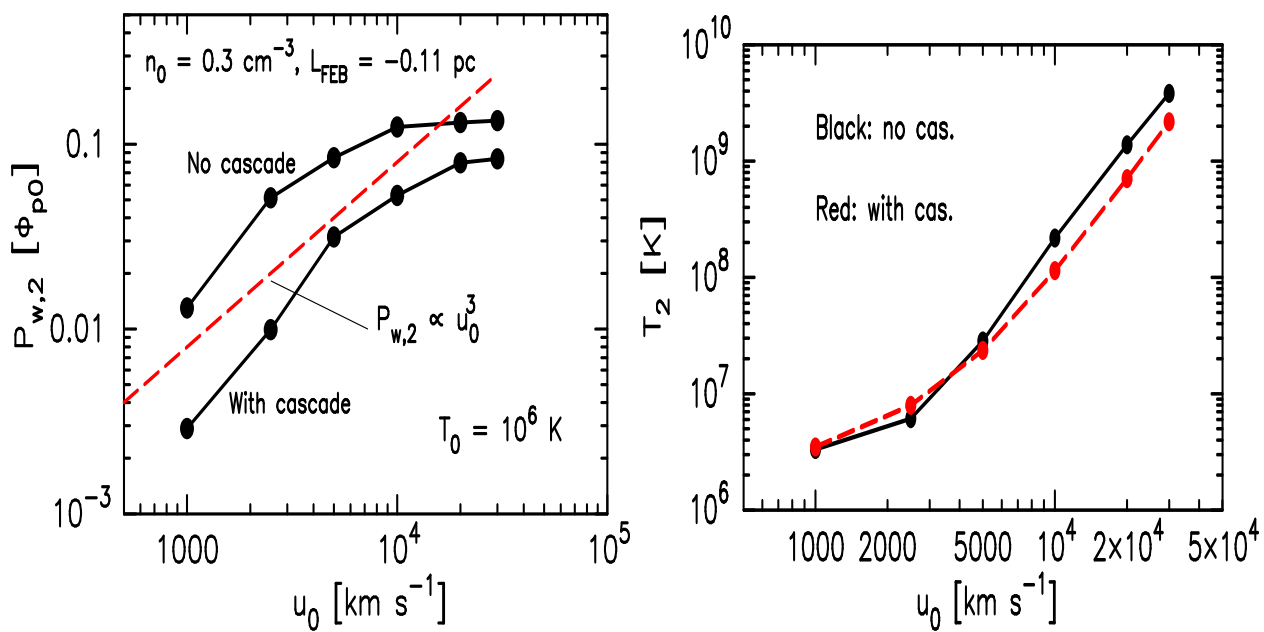

Fig. 7 Left Panel: Magnetic field amplification factor in the shock downstream $\mathrm{P}_{\mathrm{w}, 2}=$ $B_{\mathrm{t}}^{2} / 8 \pi \rho_{0} u_{0}^{2}$ as a function of shock velocity $u_{0}$ simulated in non-linear Monte Carlo diffusive shock acceleration model by Bykov et al (2014) with turbulent cascade and without turbulent cascade. Shocks propagate in plasma with initial number density $n=0.3 \mathrm{~cm}^{-3}$ and $\mathrm{T}=10^{6} \mathrm{~K}$. Right Panel: The downstream proton temperatures as a function of shock velocity for the same models as that in the left panel. The departures from the single fluid scaling $T_{2} \propto u_{0}^{2}$ evident at low velocities are due high gas compression ratio because of kinetic energy escape with CRs in the DSA models.
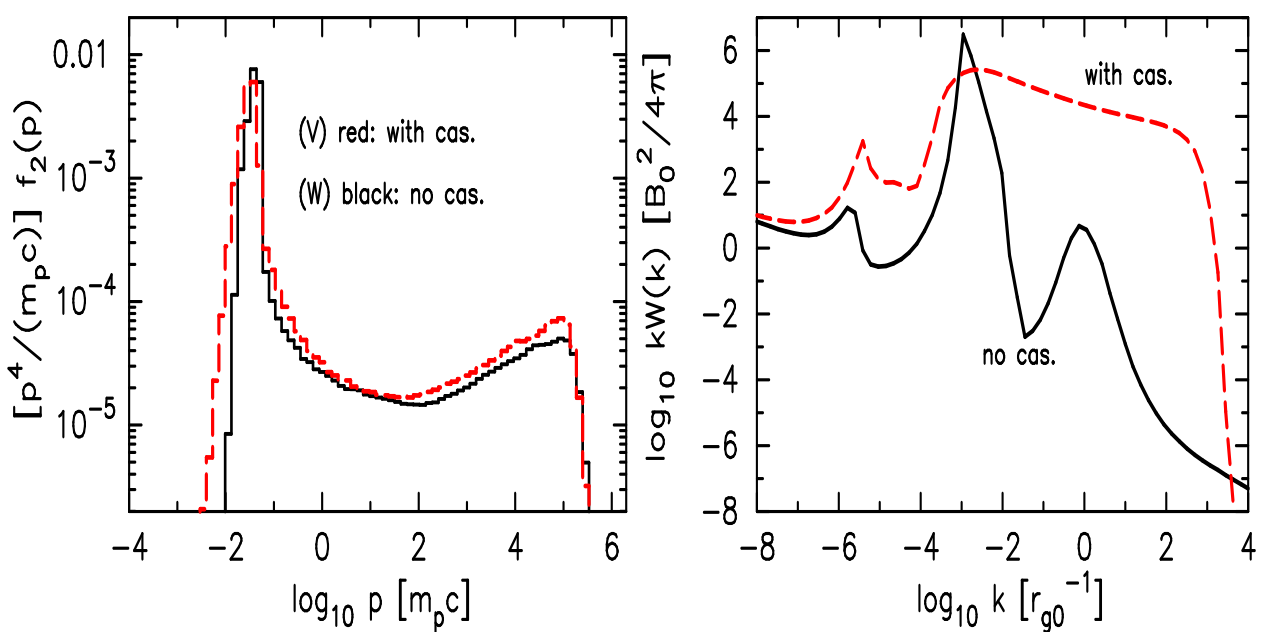

Fig. 8 Left Panel: Particle momentum distribution in the shock downstream simulated with the non-linear Monte Carlo diffusive shock acceleration model by Bykov et al (2014) with (red curve) and without turbulent cascade (black curve). Shocks of $5,000 \mathrm{~km} \mathrm{~s}^{-1}$ propagate in a plasma with initial number density $n=0.3 \mathrm{~cm}^{-3}$ and temperature $\mathrm{T}=10^{6} \mathrm{~K}$. Right Panel: Simulated magnetic field fluctuation spectra in the downstream of the same shock as in the left panel. 
instabilities on a wide range of scales, thus making the shock modeling an essentially nonlinear problem. Namely, the ponderomotive force acting on plasma due to the CR pressure gradient decelerates the upstream flow on a mesoscale which is much large than the CR gyroradii for non-relativistic shocks. Therefore the strong collisionless shock modeling is a complex multi-scale nonlinear problem. We shall not discuss here the details of diffusive shock acceleration models, referring the reader to numerous papers and reviews mentioned above, but rather illustrate in Figure 8 a simulated CR distribution and magnetic field spectra in the downstream of a shock of $5,000 \mathrm{~km} \mathrm{~s}^{-1}$ in a plasma of temperature $10^{6} \mathrm{~K}$ and number density $0.3 \mathrm{~cm}^{-3}$. The Monte Carlo simulations of non-linear diffusive shock acceleration were performed with a model account of turbulence amplification by CR-driven instabilities and some semi-phenomenological modeling of turbulence cascading. In strong shocks magnetic fluctuations are amplified by CR-driven instabilities and their magnetic spectra span a wide dynamical range of wavenumbers from macroscopic fluid scales down to the thermal ion and electron scales where the dissipation occurs. The spectra of particles and waves in the acceleration region simulated with Kolmogorov-like cascade models are shown in Figure 8 by red curves.

It is important to note that one should always be careful and distinguish energetic particles confined within an accelerator and CRs that are escaping it because they may have drastically different spectra. The problem of CR escape from accelerators is fundamentally important for the connection of the observed sources of non-thermal emission to the galactic pool of CRs and their global energetics. In popular models of diffusive shock acceleration particles mostly escape with energies close to the highest energy achieved at the given evolution stage, which is changing with time. This is a complicated non-linear problem some aspects of which were addressed in Jones and Ellison (1991), Ptuskin and Zirakashvili (2005), Reville et al (2009), Gabici et al (2009), Caprioli et al (2010), Ellison and Bykov (2011), Dwarkadas et al (2012), and Bell (2013). The fate of the rest of the confined low energy particles which are subject of adiabatic deceleration in an expanding CR accelerator at the stage when shock acceleration is slow and which determine the eventual efficiency of CR injection into the ISM has not been studied in detail yet.

The fluctuating magnetic field in the shock downstream may be highly amplified by CR-driven instabilities. A strong non-relativistic shock may transfer up to $10 \%$ of the ram pressure into magnetic fields as it is illustrated in the left panel of Figure 7. There are convincing observational evidences for strong non-adiabatic amplification of magnetic fields in the vicinities of forward shocks of a number of young SNRs including Cas A, Tycho, SN 1006, RXJ 1713.7-3946 and others obtained via arcsecond angular resolution imaging of synchrotron structures in these SNRs with Chandra (see, e.g., Vink 2012, Bamba et al 2005, Uchiyama et al 2007, Reynolds et al 2012, Helder et al 2012, Ressler et al 2014, Tananbaum et al 2014). The mechanisms of magnetic field amplification which are associated with CR-driven instabilities were thoroughly discussed and reviwed in Blandford and Eichler (1987), Bell and Lucek (2001), Malkov and Drury (2001), Zweibel (2003), Bell (2004), Lemoine and Pelletier (2010), Schure et al (2012), Blasi (2013), Bykov et al (2013a).

It should be noted that CR acceleration may strongly affect thermodynamical properties of a shock and namely, the gas compression ratio and the downstream ion temperature. The escaping CRs carry away some energy of the incoming flow 
thus increasing the gas compression and correspondingly reducing the downstream ion temperature. This is illustrated in the right panel of Figure 7. The simulations by Bykov et al (2014) also revealed that the gas compression ratio may be a nonmonotonic function of shock velocity. One should have in mind that these results were obtained for plane parallel shocks and therefore a more realistic geometry may add a factor. Also, a rigorous treatment of turbulence cascading and dissipation, which will be achievable with future numerical approaches, would allow us to check and improve current Monte Carlo models. Apart from the most popular diffusive shock acceleration, there are some models which consider multiple interactions of CRs with a shock with non-diffusive test particle transport between the encounters (see, e.g., Kirk et al 1996, Zimbardo and Perri 2013). The super-diffusive particle propagation may affect both the acceleration efficiency and the spectra of particles and magnetic turbulence. In the case of shocks propagating through partially ionized plasmas charge-exchange particle collisions provide a return flux of highly super-thermal neutrals heating the upstream plasma reducing the fluid Mach number and the compression ratio in Balmer-type shocks of velocities below $3,000 \mathrm{~km} \mathrm{~s}^{-1}$ (see, e.g., Blasi et al 2012). The efficient CR acceleration may also modify the structure and the ultraviolet-optical-infrared emission spectra of MHD radiative shocks (see, e.g., Bykov et al 2013c).

We have discussed above observational evidences for the presence of nonthermal components in colliding stellar winds, supernovae in compact clusters of massive stars, and superbubbles. All of the systems include ensembles of shocks of different strengths and likely contain strong MHD turbulence produced by fast supersonic flows in the sources of kinetic energy release. More complex flows than just isolated shocks are expected in starburst regions. Therefore, we shall discuss specific features of particle acceleration in colliding shock flows and then acceleration by multiple shocks and large scale compressive turbulence.

6.3 Particle acceleration in colliding shock flows: compact stellar clusters

The mechanism of $\mathrm{CR}$ acceleration via particle collisions with moving magnetic fields suggested by Fermi $(1949,1954)$ has a number of different realizations and it is widely used in many astrophysical applications (see, e.g., Axford 1981, Toptygin 1985, Jones 1994, Biermann 1997, Pelletier 2001). One of the most efficient versions of Fermi-type acceleration can be realized in the case of two colliding shocks driven by supersonic and super-alfvenic flows carrying magnetic fluctuations of different scales. This case is illustrated in Figure 9 by a sketch of a forward supernova shell shock approaching a termination shock of a fast wind of a massive star. At the stage when the shocks are close enough providing the distance between the shocks $\mathrm{L}_{12}$ is less then the shocks radii, the flow geometry may be approximated as plane parallel. If the flows carry magnetic fluctuations, they scatter energetic particles providing some mean free path $\Lambda(p)$ which is usually energy-dependent. The mean free path is likely larger inbetween the shocks than in a close vicinity of a shock. High energy CR particles which have mean free paths larger than $\mathrm{L}_{12}$ may cross the region between the shocks back and forth experiencing only head-on collisions with magnetic fluctuations in the approaching flows. Such particles are subject to a very efficient first order Fermi acceleration. The flow ram pressure can 

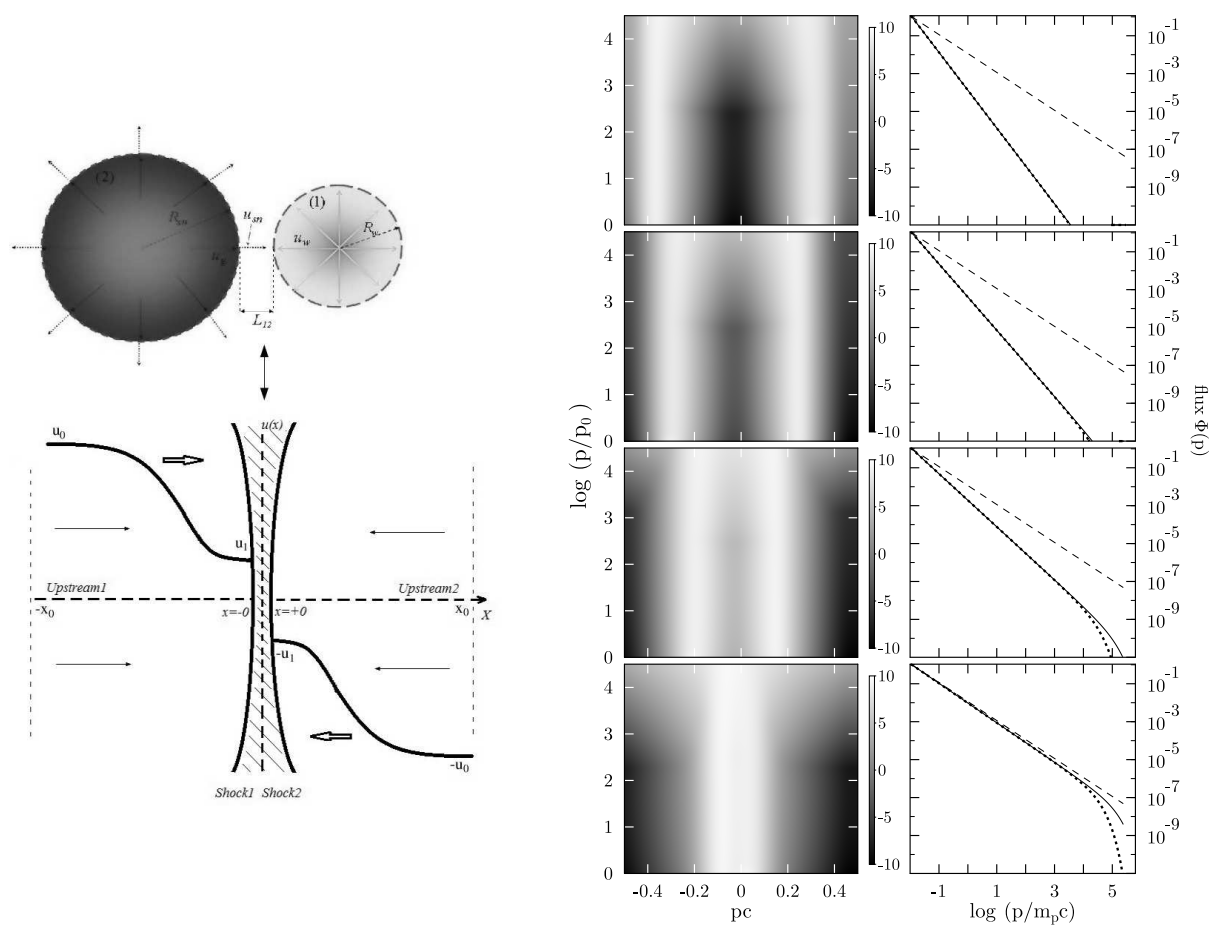

Fig. 9 Left Panel: The sketch shows an SNR shock (left circle) approaching a termination shock of a wind of a young massive star (right circle). At the stage when the shocks are close enough providing the distance between the shocks $\mathrm{L}_{12}$ is less then the shocks radii, the flow geometry may be approximated as plane parallel. High energy CR particles which have mean free paths larger than $\mathrm{L}_{12}$ may cross the region between the shocks back and forth experiencing only headon collisions with magnetic fluctuations in the approaching flows. Such particles are subject to Fermi I acceleration. Right Panel: The spatial distribution (gray scale) and differential spectra $\Phi(p)=d N / d p(p)$ of the accelerated high energy particles taken at different evolution stages simulated with a non-linear kinetic model by Bykov et al (2013b). The panels from top to bottom are corresponding to four different distances between the approaching shocks $0.6,0.5$, $0.3,0.1 \mathrm{pc}$ respectively. The proton spectrum is shown by the solid curve while the electron spectrum affected by the synchrotron and Compton losses is shown by the short dashed curve. The dotted curve shows the expected form $\Phi(p)=d N / d p(p) \propto p^{-1}$ of the time asymptotic spectrum of the protons.

be efficiently converted into the pressure of energetic non-thermal particles both confined in the accelerator and escaping the source.

The pressure of high energy particles may modify the shock velocity profiles decelerating the flows as it is shown in the bottom of the left panel of Figure 9 in the rest frame of the unperturbed ISM inbetween the shocks. On the other hand, the velocity profiles determine the spectra of accelerated particles. Therefore, the model is non-linear and the backreaction of the accelerated particles on the plasma flow is accounted for using in a semi-analytical way similar to that was developed by Malkov (1997), Blasi (2002) and Amato and Blasi (2005). This model assumes a Bohm-like recipe of diffusive propagation of CR particles in the close vicinity of the shocks, supposing a high level of CR-driven instabilities amplified 
by magnetic fluctuations (e.g., Bell 2004, Schure et al 2012, Bykov et al 2014), while the mean free path of the highest energy particles in between the shocks is larger than $\mathrm{L}_{12}$. In this system particle distribution is nearly isotropic even for high energy particles with $\Lambda(p) \gtrsim L_{12}$ and the kinetic equation reduces to the so-called telegraph equation.

The right panel of Fig. 9 shows the results of non-linear time-dependent modeling of energetic particle distribution in the vicinity of two closely approaching fast plane-parallel MHD shocks at different distances between the shocks (from the top to bottom) as it was simulated by Bykov et al (2013b). The gray scale panels in Fig. 9 illustrate the spatial distribution of particles of different energies at different moments of time. The proton spectra are approaching the asymptotic power-law distribution, which corresponds to a very hard spectrum $N(\gamma) \propto \gamma^{-1}$ before the break (the dotted line in the far right spectral panels). The electron spectrum in this simulation is similar to that of proton, but has a maximal energy below that of the protons because of the synchrotron and inverse Compton energy losses. The electrons and positrons in this case are confined within the accelerator and only a fraction of high energy protons can escape the system. If the synchrotron or Compton losses are not strong enough then the highest energies of electrons and protons are similar and electrons might escape the accelerator as well.

The hard spectra of the accelerated particles confined in the acceleration zone result in very hard emission spectra with rather sharp breaks of high energy radiation both of leptonic and hadronic origin. The modelled objects may appear as "dark accelerators" with a peaked spectral energy distribution of the high energy non-thermal radiation, but no apparent counterparts at longer wavelengths. The protons which escape the accelerator at different stages of the system evolution and produce pions via collisions with the gas in the surrounding dense shells or clouds may radiate spectra of various hardness depending on the time evolution of their maximal energy. It is important to note that the spectra of escaped protons integrated over the lifetime of the accelerator are typically significantly softer than the spectra of particles confined within the source and may have spectral indexes close to -2 . The exact value of the spectral index of the escaped particles which are contributing to the diffuse galactic CR population depends on the details of the complex evolution of the source.

In the case of a supernova shock of velocity $u_{s}$ approaching a fast wind of a massive star of velocity $u_{w}$ in Bohm diffusion regime with $\Lambda(p) \approx R_{g}(p)$, the particle acceleration time $\tau_{a}$ can be estimated as

$$
\tau_{a}(\gamma) \approx \frac{c R_{g}(\gamma)}{u_{s} u_{w}}
$$

where $R_{g}(\gamma)$ is the gyro-radius of a CR particle. The scale of the magnetic field amplified by CR-driven instabilities in this system can be approximated as $B \approx$ $\sqrt{4 \pi \eta_{b} \rho} u_{s}$, where the efficiency of magnetic field amplification $\eta_{b} \sim 0.1$ (c.f. the left panel in Fig. 7) and $\rho \approx m_{p} n$ is the ambient density. Then the acceleration time is $\tau_{a} \approx 2 \cdot 10^{10} \gamma_{6}\left(\eta_{b} n\right)^{-0.5} u_{s 3}^{-2} u_{w 3}^{-1} \mathrm{~s}$, where $u_{s 3}$ and $u_{w 3}$ are measured in the units of $10^{3} \mathrm{~km} \mathrm{~s}^{-1}$ and CR proton Lorentz factor $\gamma_{6}$ is in the units of $10^{6}$. The free expansion phase lasts about 250 yrs $\left(M / \mathrm{M}_{\odot}\right)^{5 / 6} n^{-1 / 2} E_{51}^{-1 / 2}$, where the velocity of an ejected mass $M$ is constant at about $10^{4}\left(M / \mathrm{M}_{\odot}\right)^{-1 / 2} E_{51}^{1 / 2} \mathrm{~km} \mathrm{~s}^{-1}$. 
6.4 Neutrinos and gamma-rays from compact clusters

In the case of a young supernova shock propagating through the winds of massive stars in the compact cluster Westerlund I where $n \sim 0.6 \mathrm{~cm}^{-3}$ (Muno et al 2006b), one can estimate $\tau_{a} \approx 400$ yrs for a proton of energy about $40 \mathrm{PeV}$, if $u_{s 3} \sim 10$ and $u_{w 3} \sim 3$. The high velocity of a supernova shock $u_{s 3} \sim 10$ is expected at the free expansion stage if the mass of the fast ejecta is about of a few solar masses. Therefore, the maximal energy of the protons accelerated at a few hundred years old supernova shock approaching a fast wind of a massive star may reach $20-40 \mathrm{PeV}$ and thus, supernovae in compact clusters of massive stars should be considered as potential "PeVatron" sources. It should be noted that the estimations above are rather optimistic since the real geometry of such a system is likely to be more complicated. However, if a supernova explosion occurs a few parsecs outside of the core of a cluster of massive stars, the geometry factors might be alleviated since the supernova shock may collide with the cluster-scale wind of $u_{w 3} \gtrsim 3$.

The efficient CR acceleration in the colliding shock flow lasts for about a few hundred years when a substantial part of the mechanical power of the supernova shell is transferred to $\mathrm{PeV}$ energy CRs with a very hard spectrum. The spectra of gamma-ray and neutrino emission from CRs inside the acceleration zone have to be derived with an account for both the pion decay hadronic emission and the inverse Compton components. The corresponding radiation cross sections can be found in ( e.g., Aharonian 2004, Kelner et al 2006, Kamae et al 2006). During this relatively short stage the accelerator can be detected as a source of high energy radiation of both hadronic and leptonic origin. For the distant sources of $\mathrm{PeV}$ emission the gamma-ray opacity of the extragalactic background light must be also accounted for (see, e.g., Aharonian 2004, Dwek and Krennrich 2013). At the later stage the CRs initially confined in the accelerator are released and the escaped particles diffuse through the ambient ISM producing $\mathrm{PeV}$ regime photons and neutrinos due to hadronic CR interactions.

In Fig. 10 we show the simulated spectra of neutrino and gamma-ray emission produced by the CRs escaped from the accelerator and diffusing into the surrounding cloudy medium. PeV regime particles are expected to diffuse to distances $\gtrsim$ 30 pc from the source in $\lesssim 10,000$ yrs after the release from the acceleration site. The diffusion time roughly corresponds to the estimated age of the supernova which has produced the magnetar CXOU J1647-45 discussed in $\S 3.4$, because the lifetime of the accelerator is much shorter. A possible "PeVatron" source at the distance of Westerlund I simulated with the nonlinear CR acceleration model of Bykov et al (2013b) for the case of a supernova shock of velocity $u_{s 3} \sim 10$ at the free expansion stage colliding with a cluster scale wind of $u_{w 3} \sim 3$ as it was discussed above. The data points were taken from the H.E.S.S. observations by (Abramowski et al 2012a). We do not discuss here the Fermi-LAT source found in the vicinity of Westerlund I since Ohm et al (2013) attributed the GeV emission to a pulsar wind nebula. Supernovae interacting with fast winds of massive star clusters are plausible sources of $\sim 40 \mathrm{PeV}$ protons and $\mathrm{PeV}$ regime gamma-ray emission and neutrinos, produced by the CRs in p-p collisions with shock-compressed ambient medium. This can possibly explain some of the neutrino events detected recently by the Ice Cube Observatory (Aartsen et al 2013, IceCube Collaboration et al 2013a, Aartsen et al 2014) which are clumped within three median angular errors 
from the direction to Westerlund I and the surrounding clouds associated with the TeV source detected by H.E.S.S. ${ }^{1}$

Diffusive shock acceleration in isolated SNRs is a likely mechanism to accelerate the bulk of the galactic cosmic rays up to PeV regime energies (Hillas 2005. Bell et al 2013, Amato 2014). However, the type IIb supernovae, a subclass which comprises likely about $3 \%$ of the observed core collapsed $\mathrm{SNe}$ rate, was argued by Ptuskin et al $(2010)$ to be able to produce CRs of energies $\sim 100 \mathrm{PeV}$ at the early stages of SNR evolution. This type of sources may be in competition with SNR - wind accelerators, which we have discussed above. The sources of nonthermal emission and CRs in compact stellar clusters may contribute to galactic $\mathrm{CR}$ spectrum in the $\mathrm{PeV}$ regime and above. Some spectral features above the spectral knee indicated by recent observations (see, e.g., IceCube Collaboration et al 2013b, Berezhnev et al 2013, Apel et al 2013) may be attributed to individual contributions from some of the sources associated with the compact stellar clusters.

Apart from a few parsec scale winds from the compact stellar clusters discussed above, a larger scale wind outflow of velocity $u_{w 3} \lesssim 0.3$ was revealed in the Galactic Center region (see, e.g., Bland-Hawthorn and Cohen 2003, Law 2010, McClure-Griffiths et al 2013, Carretti et al 2013). The large scale outflow from the Galactic Center region is likely connected to the recently discovered "Fermi bubbles" Su et al (2010) and to the two giant radio lobes (Carretti et al 2013). Supernova remnants in the Galactic Center region may collide with the wind producing radio and gamma-ray sources with flat spectra which may account for some of the filamentary non-thermal radio structures with flat spectra observed within the inner $\sim 100 \mathrm{pc}$ vicinity of the Galactic Center (see, e.g., Morris and Serabyn 1996, Yusef-Zadeh 2003).

$\mathrm{PeV}$ neutrino sources with hard spectra may contribute to starburst galaxy high energy radiation. CR interactions in starburst galaxies are expected to produce efficiently high energy gamma-rays and neutrinos. Loeb and Waxman (2006) predicted that the cumulative contribution of starburst galaxies would produce a measurable neutrino background well above that is expected from atmospheric neutrino production.

6.5 Particle acceleration by long-wavelength turbulence and multiple shocks: superbubbles

Particle acceleration by individual SNRs and colliding shock flows may occur on time scales well bellow 1,000 yrs. However, as discussed in $\S$, an OB star association on the time scales above a few hundred thousand years may enter the evolution stage with multiple SN explosions, which create large caverns of $\gtrsim$ 50 pc size - galactic superbubbles. The injected mechanical power may reach $\sim 10^{38} \mathrm{erg} \mathrm{s}^{-1}$ over $\gtrsim 10 \mathrm{Myr}$ - the lifetime of a superbubble . This process is accompanied by formation of multiple shocks, large scale flows and broad

\footnotetext{
1 After this review was published the model of Westerlund I described above was refined and the detailed version with comparison to 3 years Ice Cube data is published in "Ultrahard spectra of $\mathrm{PeV}$ neutrinos from supernovae in compact star clusters" (MNRAS v. 453, p.113$121,2015)$. Therefore I updated in this arxive version the Figures 10-12 which are adopted from the paper.
} 


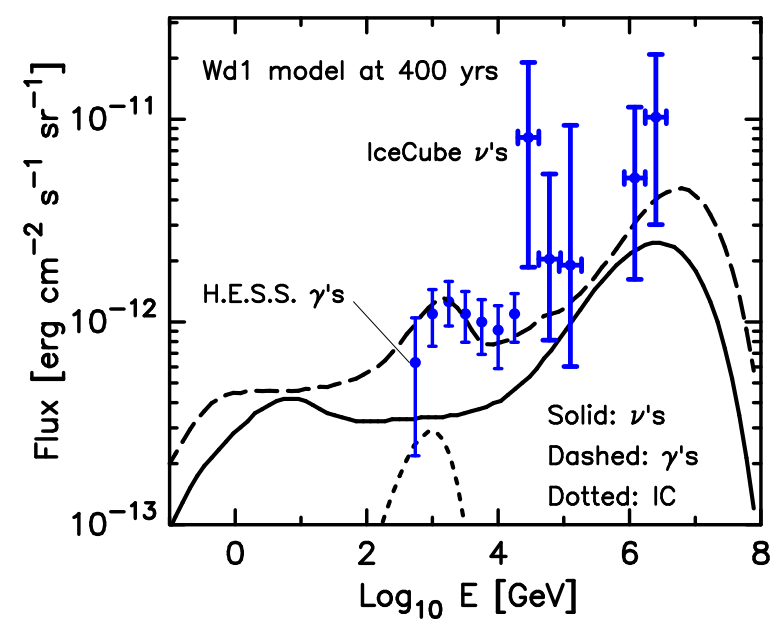

Fig. 10 Spectra of the neutrino and gamma-ray emission from a PeVatron source simulated in a model of particle acceleration by a free expanding shock of a supernova remnant colliding with a wind of a compact cluster of massive stars. Both the neutrino (solid line) and gamma-ray emission spectra (dashed line) are from the pion decays produced due to inelastic $p p$ collisions of protons accelerated by the short lived accelerator with the surrounding clouds. The spectra of particles accelerated by colliding shocks may be significantly harder than that accelerated by young isolated supernova remnants. This results in the apparent $\mathrm{PeV}$ regime spectra upturn. For illustration purposes we put the source to the distance of the compact cluster Westerlund 1 and the data points are from H.E.S.S. observations of Westerlund 1 by Abramowski et al (2012a). The data points for the H.E.S.S. source, and the five Ice Cube events explained in Fig. 12 are presented here just to illustrate how they compare to the model predictions when the source is $\sim 400 \mathrm{yr}$ old and point-like, i.e., as we expect Westerlund I was about $10^{4}$ years ago. The simulated gamma-ray and neutrino emission at the present time from CRs that escaped the accelerator in Westerlund $\mathrm{I} \sim 10^{4} \mathrm{yr}$ ago are summarized in Figs. 11 and 12 The figure is adopted from "Ultrahard spectra of $\mathrm{PeV}$ neutrinos from supernovae in compact star clusters" (MNRAS v. 453, p.113-121, 2015).

spectra of MHD fluctuations in a tenuous plasma with frozen-in magnetic fields. The collective effect of multiple SNRs and strong winds of young massive stars in a superbubble may be able to re-energize CR particles (see Montmerle 1979 , Cesarsky and Montmerle 1983, Bykov and Fleishman 1992, Axford 1994, Higdon et al 1998, Klepach et al 2000, Bykov and Toptygin 2001, Bykov||2001, Ramaty et al 2001, Parizot et al 2004, Marcowith et al 2005, Ferrand and Marcowith 2010) and even to extend the spectrum of accelerated particles to energies well beyond the Knee (Bykov and Toptygin 2001).

Nonthermal particles in superbubbles are scattered by small scale magnetic fluctuations which determine their diffusive mean free paths $\Lambda(p)$, and also experience multiple interactions with shocks and large scale (i.e., of scales above $\Lambda(p)$ ) compression and rarefaction waves. Estimations of the mean free paths of super-thermal particles depend on rather uncertain hypothesis on the strength, polarization and statistical properties of small scale magnetic fluctuations which are produced either via cascading power from the stirring scales down to the scales of thermal gyroradii or by small scale instabilities of a local flow. Even in a single 


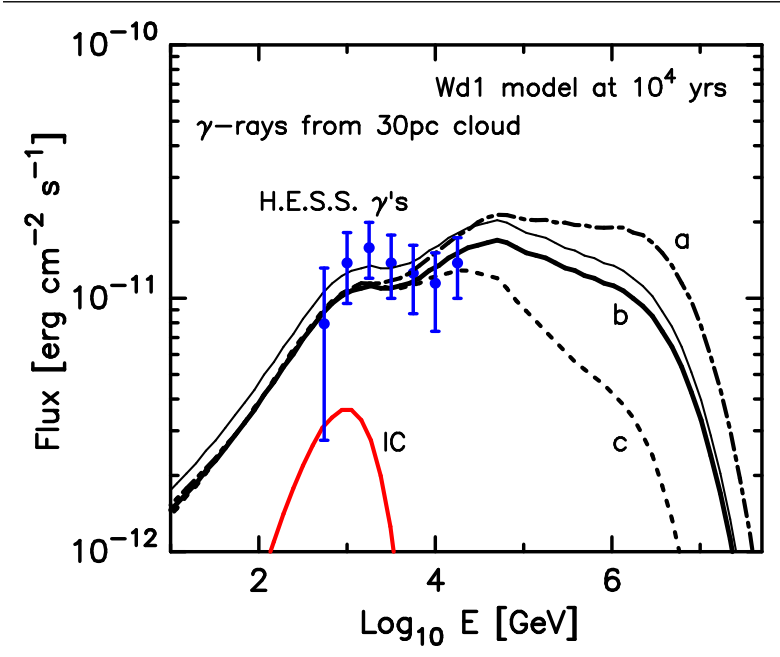

Fig. 11 Gamma-ray emission from inelastic $p-p$-interactions in the CSF source at $\sim 10^{4} \mathrm{yr}$ after the SN explosion when CR protons produced in the short-lived accelerator have propagated into a nearby cloud of $\sim 30 \mathrm{pc}$ size. The magnetic field amplified by the CR-driven instabilities in the vicinity of the fast shock in the CSF accelerator were parameterized as 0.8 $\mathrm{mG}$ (c), $0.9 \mathrm{mG}$ (b), and $1 \mathrm{mG}$ (a), all below $10 \%$ of the ram pressure. The IC curve is inverse Compton emission from the secondary electrons produced by the inelastic $p-p$ interactions in the cloud. Only the $\gamma$-rays from the H.E.S.S. field of view are included. The gas number density of the nearby cloud is $25 \mathrm{~cm}^{-3}$, except for the light-weight solid curve where it is $30 \mathrm{~cm}^{-3}$ with $B=0.9 \mathrm{mG}$. The figure is adopted from "Ultrahard spectra of $\mathrm{PeV}$ neutrinos from supernovae in compact star clusters" (MNRAS v. 453, p.113-121, 2015).

fluid MHD models cascading and dissipation in magnetic turbulence are rather complex processes (see, e.g., Kolmogorov 1941, Biskamp 2008, Schekochihin et al 2009, Brandenburg and Nordlund 2011), where a transition from collisional to collisionless plasma regimes occur, as it is the case in the starburst environment.

These processes are a lot more complex in the presence of cosmic rays. CRdriven instabilities strongly affect the turbulent dynamics at the mesoscopic gyrokinetic scales which span the range between the gyroradii of energetic particles and the scales of the energy-containing magnetohydrodynamic plasma (see Bykov et al 2013a, and references therein). The CR-driven instabilities result in crossscale coupling in the turbulence. In the lack of rigorous models of the magnetic turbulence one can rely on direct observations of collisionless turbulence in the Earth's magnetosphere and in the heliosphere (Matthaeus and Velli 2011, Dudok de Wit et al 2013) where the anisotropic magnetic fluctuations with piece-wise power-law spectra are measured down to the electron gyroradius scale (Tu and Marsch 1995, Leamon et al 1998, Bale et al 2005, Alexandrova et al 2013). Given the complexity of the multi-scale flows and the lack of the observational data on the different scales, a practical way to treat particle acceleration processes in starburst regions and superbubbles on $\sim$ Myr time scale is to use coarse-grained kinetic equations which are consequently averaged over scales ranging from particle microscopic gyro-scales up to the macroscopic hydrodynamical scales determined by the repeated shock waves produced by supernovae and stellar winds. Within 


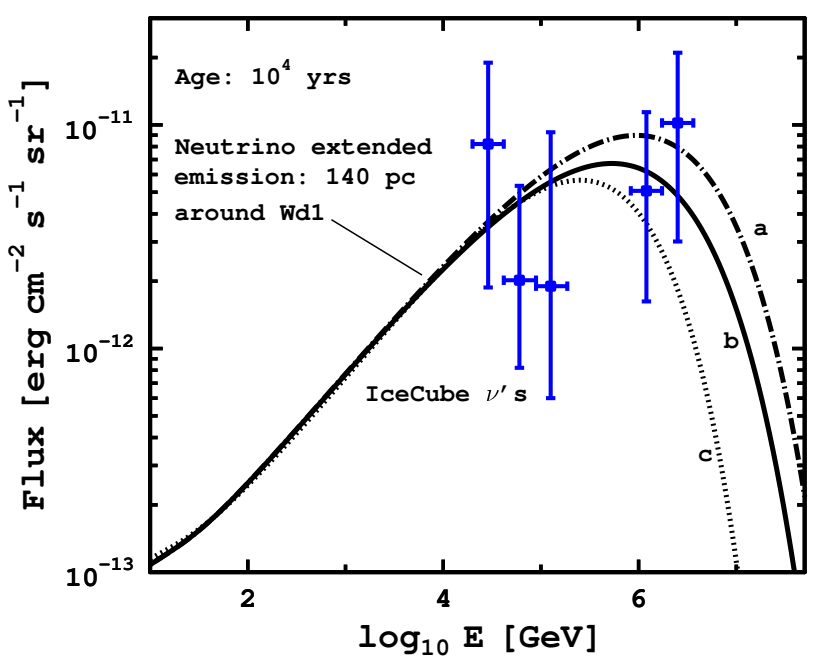

Fig. 12 Neutrino emission from an extended $(\sim 140 \mathrm{pc}$ radius $)$ source $\sim 10^{4} \mathrm{yr}$ after the SN explosion when CRs produced in the short-lived accelerator have propagated into the surrounding material. The amplified magnetic fields in the short-lived acceleration source which determine the maximal energy of accelerated protons are $0.8 \mathrm{mG}$ (in the curve c), $0.9 \mathrm{mG}$ (in the curve b), and $1 \mathrm{mG}$ (in the curve a). We note that the gamma-rays in Figs. 11 and the neutrinos here originate from different volumes: only the $\gamma$-rays from the H.E.S.S. field of view are shown in Fig. 11] while the neutrinos are from a larger region of radius $140 \mathrm{pc}$. The neutrino data points ( $1 \sigma$ energy flux error bars) are a subset of the five events in the vicinity of Westerlund I from 37 Ice Cube events of the first 3 years (Aartsen et al 2014)). The figure is adopted from "Ultrahard spectra of $\mathrm{PeV}$ neutrinos from supernovae in compact star clusters" (MNRAS v. 453, p.113-121, 2015).

this approach the CR distribution function $F(\boldsymbol{r}, p, t)$ satisfies a kinetic equation subsequently averaged over an ensemble of fluctuations of electric and magnetic fields induced by turbulent motions of highly conductive plasma with shocks of different strengths which introduce intermittency into the system.

A statistical description of non-thermal particle distribution in intermittent systems with long wavelength compressible motions and multiple shocks was developed by Bykov and Toptygin (1993) who obtained a kinetic equation for the mean distribution function away from the strong shocks in the form

$$
\frac{\partial F}{\partial t}-\frac{\partial}{\partial r_{\alpha}} \chi_{\alpha \beta} \frac{\partial F}{\partial r_{\beta}}=G \hat{L} F+\frac{1}{p^{2}} \frac{\partial}{\partial p} p^{4} D \frac{\partial F}{\partial p}+A \hat{L}^{2} F+2 B \hat{L} \hat{P} F+Q_{j}(p),
$$

the source term $Q_{j}(p)$ is determined by injection of the nuclei of a type $j$. The integro-differential operators $\hat{L}$ and $\hat{P}$ are given by

$$
\hat{L}=\frac{1}{3 p^{2}} \frac{\partial}{\partial p} p^{3-\delta} \int_{0}^{p} \mathrm{~d} p^{\prime} p^{\prime}{ }^{\delta} \frac{\partial}{\partial p^{\prime}} ; \quad \hat{P}=\frac{p}{3} \frac{\partial}{\partial p} .
$$

The index $\delta$ of the CR momentum distribution at a strong shock in the test particle case is about 4, but in non-linear models it may differ from this universal value. 


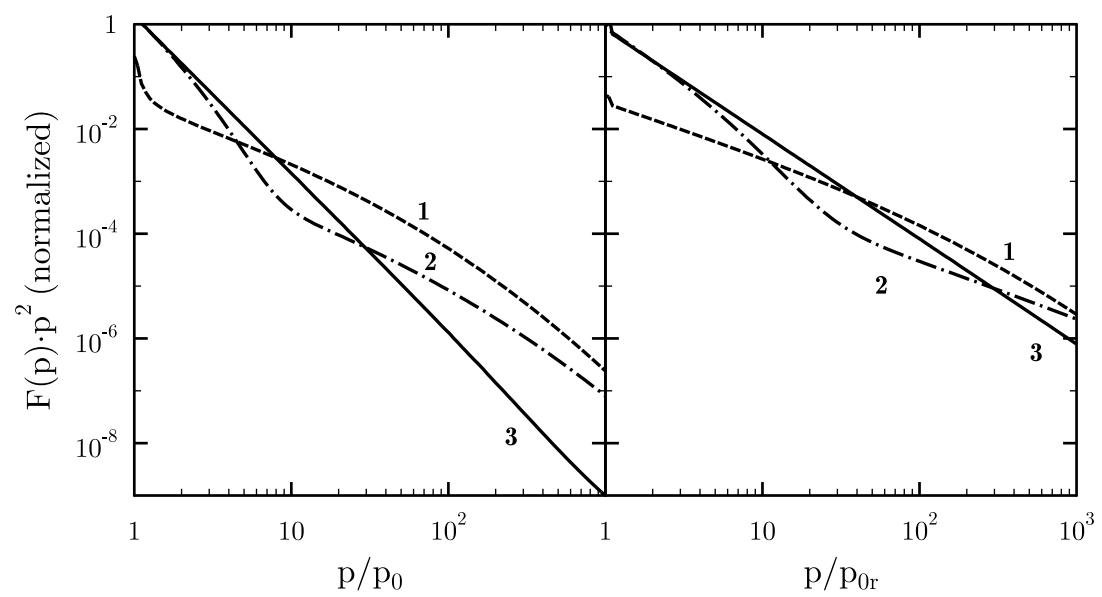

Fig. 13 Temporal evolution of a particle distribution function in a superbubble simulated with the non-linear model of low-energy CR acceleration by Bykov (2001). Monoenergetic injection was assumed with the injection energies of about $10 \mathrm{keV}$ (left panel) and $1 \mathrm{GeV}$ (right panel). The turbulence energy-containing scale was $10 \mathrm{pc}$ and the r.m.s. velocity amplitude was 1,000 $\mathrm{km} \mathrm{s}^{-1}$ for a superbubble of $50 \mathrm{pc}$ size. The acceleration time was about $3 \times 10^{5} \mathrm{yrs}$. Curves 1,2 , and 3 show the CR spectra at 4, 6, and 10 acceleration times, correspondingly.

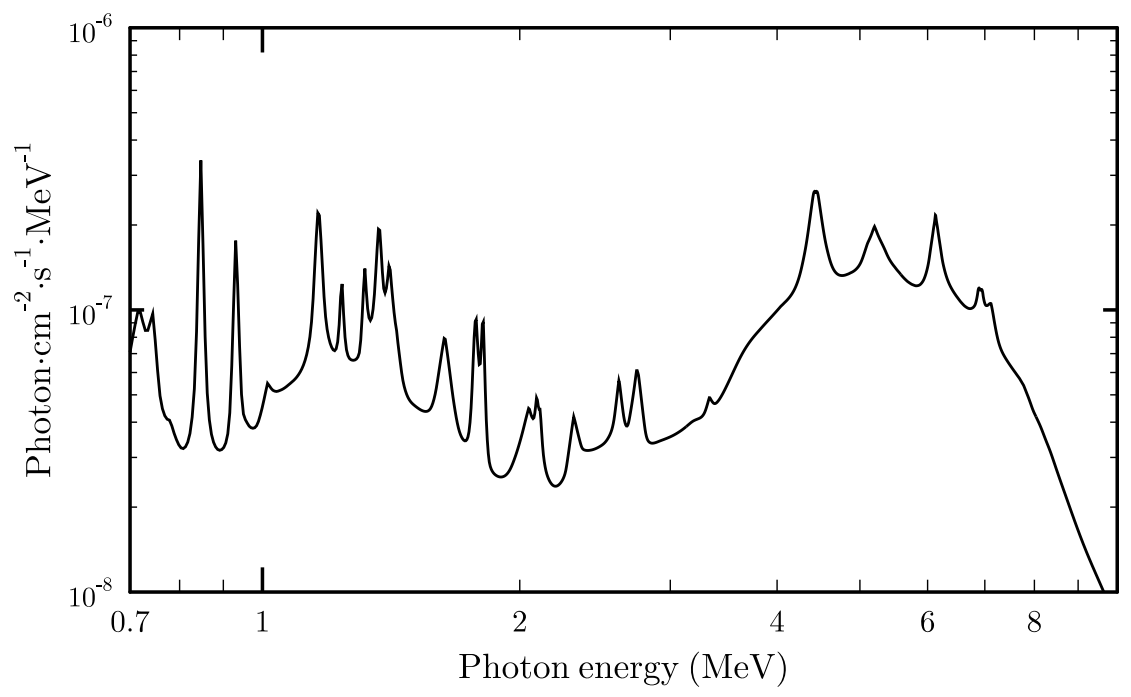

Fig. 14 A simulated spectrum of nuclear interaction gamma-ray line emission for a superbubble of a scale size of about $50 \mathrm{pc}$ of the age $\sim$ Myr simulated with low energy CR particle spectra given in left panel Fig. 13 
The information on the turbulence and shocks is in the kinetic coefficients $A$, $B, D, G, \tau_{s h}$, and diffusion tensor $\chi_{\alpha \beta}$ which were expressed in terms of spectral functions describing correlations between shocks and the long wavelength (i.e., of scales $l \gg \Lambda(p))$ turbulent motions of compressible plasma. Short scale magnetic fluctuations determine the local CR scattering rate and therefore, the mean free path $\Lambda(p)$ and its momentum dependence. The kinetic coefficients satisfy some transcendental renormalization equations given in Bykov and Toptygin (1993)and Bykov (2001) which connect these to the transverse and longitudinal parts of the Fourier components of the turbulent velocity correlation tensor and the correlations between velocity jumps on shocks and also between shocks and smooth rarefactions.

Two distinct regimes of CR acceleration in a turbulent bubble characterized by an energy-containing scale $l$ with the amplitude of the bulk plasma speed $u$ should be recognized. At low enough CR energies of $p<p_{*}$, where $\Lambda\left(p_{*}\right)=l u / v$, the CR transport is determined by turbulent advection (here $v$ is the CR particle velocity). The diffusion tensor $\chi_{\alpha \beta}$ is nearly momentum independent. CR particles are strongly coupled to plasma motions, CR acceleration time is close to the turbulence turnaround time and particles may strongly affect the the supersonic turbulence. In a nonlinear model by Bykov (2001) where a simplified description of the long-wavelength turbulence was considered, an efficient conversion of the shock turbulence power into CRs of momenta $p<p_{*}$ and a strong time evolution were found for particles injected initially with the same momentum $p_{0}<p_{*}$. In nonlinear models no superposition principle holds and therefore, the distribution function calculated for monoenergetic injection can not be used as a Green function.

In Figure 13 the evolution of CR distribution is illustrated with two models. In the first model shown in the left panel the proton injection momentum $p_{0}$ was chosen to be non-relativistic (a few $\mathrm{keV}$ ). The CR spectra evolution in this nonlinear model follow soft-hard-soft scenario with time asymptotic power-law momentum distribution of non-relativistic particles $p^{2} F(p) \propto p^{-3}$ (curve 3 in the left panel in Fig. 13). After an appropriate scaling this case can be confronted against satellite observations of low energy $\mathrm{CR}$ population in the corotation interaction regions of the heliosphere, where multiple shocks and compressive waves accelerate particles.

Analysing quiet epoch suprathermal ion distributions in the heliosphere Fisk and Gloeckler (2006, 2012) pointed out that the observed suprathermal tails follow power laws $\propto v^{-5}$ and the spectral slope of index " -5 " is universal in this objects. The quiet epoch spectra should correspond to our mean distribution function, while in shock vicinities the spectra are harder. The universal index " 5 " exactly corresponds to asymptotic power-law $p^{2} F(p) \propto p^{-3}$ in Fig. 13 . Long wavelength compressible motions are likely to play an important role in shaping CR distributions in the interplanetary medium (Fisk and Gloeckler 2012, Jokipii and Lee 2010, Zhang and Lee 2013, Antecki et al 2013). The low energy CR spectra in a superbubble could be tested with gamma-ray line observations in $\mathrm{MeV}$ regime. In the right panel in Figure 14 we show a simulated spectrum of gamma-ray line emission which could be detected from a superbubble at a few kpc distance with $\mathrm{MeV}$ regime detectors of sensitivity $\sim 10^{-7} \mathrm{ph} \mathrm{cm}^{-2} \mathrm{~s}^{-1}$ which are not available yet.

In the case of relativistic CR injection, but still in the regime of low energy CRs, where $p<p_{*}$, the proton spectrum in Fig. 13) again demonstrates a soft- 

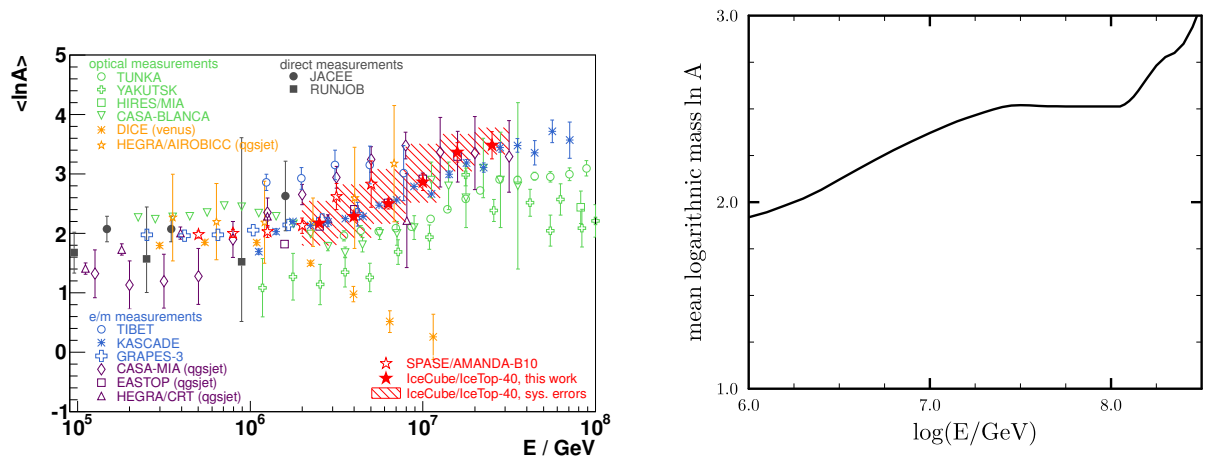

Fig. 15 Left Panel: The mean logarithm mass of galactic cosmic rays as observed by different detectors and compiled by IceCube Collaboration et al (2013b). Right Panel: The mean logarithm mass of CRs accelerated in a superbubble as simulated by Bykov and Toptygin (2001).

hard-soft behavior, but the asymptotic power-law distribution is $p^{2} F(p) \propto p^{-2}$ which is harder than that in the non-relativistic case. The pion production in $p p$ collisions of the protons in the energy range between $\mathrm{GeV}$ and $\mathrm{TeV}$ regimes accelerated in a superbubble may explain the $\mathrm{GeV}-\mathrm{TeV}$ emission from the Cygnus cocoon discovered with Fermi-LAT by Ackermann et al (2011) and shown in Fig. 4 (see also The ARGO-YBJ Collaboration et al 2014). Somewhat softer observed gamma-ray spectra may be attributed to the elongated shape of the cocoon, as only a spherical superbubble was modelled. The elongated shape would alleviate CR escape from the cocoon resulting in softer spectra. The value of the maximal momentum $p_{*}$ depends on the amplitude of small scale collisionless turbulence which can not be resolved and can be estimated only indirectly, e.g., from electron density fluctuations derived from the observed interstellar scintillation of pulsar signals (see, e.g., Armstrong et al 1995). One can expect the transition energy $c p_{*}$ to be at $\mathrm{TeV}$ regime in an extended superbubble. A possible presence of a 'kink' in CR particle spectra at about $0.2 \mathrm{TeV}$ per nucleon found in direct measurements of galactic CRs was attributed by Erlykin and Wolfendale (2012) to propagation and acceleration processes in the Local Bubble.

High energy ions of $p>p_{*}$ accelerated in superbubbles may contribute to observed distribution of galactic cosmic rays even at energies above the Knee where a substantial enrichment with heavy ions is expected in the superbubble model. In the right panel of Figure 15 we present the mean atomic weight of CRs as a function of CR energy as it was simulated by Bykov and Toptygin (2001). This can be compared to the recent compilation of observations available from IceCube Collaboration et al (2013b). Models of CR acceleration in superbubbles were argued by Higdon and Lingenfelter $(2005)$ as a favourable way to explain the anomalous ${ }^{22} \mathrm{Ne} /{ }^{20} \mathrm{Ne}$ ratio in CRs (c.f. Casse and Paul (1982)), the cosmic-ray actinide/Pt group and $\mathrm{UPuCm} / \mathrm{Th}$ ratios, and the constant $\mathrm{LiBeB} /(\mathrm{C}+\mathrm{O})$ ratio observed in very old, metal-poor stars (see also Parizot et al 2004, Binns et al 2008). However, Prantzos (2012) has recently argued against the superbubble model of the ${ }^{22} \mathrm{Ne} /{ }^{20} \mathrm{Ne} \mathrm{CR}$ isotope anomaly, thus further investigation of the superbubble models of CR origin is needed. 


\section{Concluding remarks}

A significant progress has been recently made in detection and investigation of gamma-ray spectra of starburst galaxies M82, NGC 253, NGC 1068 with the Large Area Telescope aboard Fermi gamma-ray observatory and imaging atmospheric Cherenkov telescopes H.E.S.S., MAGIC, and VERITAS. The observations revealed the presence of efficient mechanisms of particle acceleration at least up to the $\mathrm{TeV}$ range. For the first time gamma-ray emission was detected from the extended cocoon associated with OB-star complexes in Cygnus X, which may represent the class of galactic superbubbles. High resolution X-ray imaging and spectroscopy of compact clusters of massive stars Westerlund I, Arches, Quintuplet and the superbubbles in LMC with Chandra, XMM-Newton, INTEGRAL, and NuSTAR $\mathrm{X}$-ray observatories provided clear evidences of multi-component emission which is produced by thermal and non-thermal sources due to violent processes of wind collisions and to compact remnants of supernovae.

In general, processes of particle acceleration in the violent starburst regions are due to efficient conversion of kinetic power of the fast outflows of winds from massive stars and supernovae into energetic particles and fluctuating magnetic fields, which occurs in the vicinities of fast shocks and colliding supersonic flows. The efficiency of kinetic power conversion into cosmic rays may reach tens of percent which indicates a substantial backreaction of the accelerated particles on the flow dynamics. Therefore, MHD modeling of plasma heating and large scale wind formation in compact clusters of massive stars and superbubbles should account for the pressure of non-thermal particles and magnetic fields.

Nonlinear models of energetic particle acceleration discussed in this review illustrate the diversity of the acceleration process. Supernova shells colliding with a cluster wind may give birth to compact sources with hard spectra of $\mathrm{PeV}$ regime CRs accelerated on a time scale shorter than 1,000 yrs. The subsequent escape of the accelerated particles should produce extended sources of gamma-rays and neutrinos. This can be tested with the next generation of Cherenkov telescopes and with the existing neutrino telescopes like the South Pole neutrino observatory Ice Cube.

CRs may be efficiently accelerated and confined in superbubbles on Myrs time scale. The long lived superbubbles may contain a low-energy CR component, whose partial pressure is substantial. The low-energy component can be constrained via a search for $\mathrm{MeV}$ range gamma-ray lines, which would require a new generation of sensitive spectrometers to appear. Being an energetically significant the long-lived CR component produced during collisionless relaxation of violent plasma outflows in starburst environments provide ionization of the starforming clouds and thus it is an important integral part of galactic ecology.

Acknowledgements The invitation to this review by T. Courvoisier is deeply acknowledged. The author benefited from his collaborations with A. Artemyev, C. Georgy, I. Grenier, D.C. Ellison, G. Fleishman, P. Gladilin, M. Gustov, A. Marcowith, K.P. Levenfish, D. Folini, S. Osipov, E. Parizot, Yu. Uvarov, R. Walder. Special thanks are due to I.N. Toptygin and A.M. Krassilchtchikov for their help at various stages of my work. 


\section{References}

Aartsen MG, Abbasi R, Abdou Y, Ackermann M, Adams J, Aguilar JA, Ahlers M, Altmann D, Auffenberg J, Bai X, et al (2013) First Observation of PeV-Energy Neutrinos with IceCube. Physical Review Letters 111(2):021103, DOI 10.1103/PhysRevLett.111.021103

Aartsen MG, Ackermann M, Adams J, Aguilar JA, Ahlers M, Ahrens M, Altmann D, Anderson T, Arguelles C, Arlen TC, et al (2014) Observation of High-Energy Astrophysical Neutrinos in Three Years of IceCube Data.Physical Review Letters 113(10):101101, DOI 10.1103/ PhysRevLett.113.101101

Abbott DC (1982) The theory of radiatively driven stellar winds. II - The line acceleration. Astrophys J 259:282-301, DOI 10.1086/160166

Abdo AA, Ackermann M, Ajello M, Atwood WB, Axelsson M, Baldini L, Ballet J, Band DL, Barbiellini G, Bastieri D, et al (2009) Fermi/Large Area Telescope Bright Gamma-Ray Source List. Astrophys J Suppl 183:46-66, DOI 10.1088/0067-0049/183/1/46, 0902.1340

Abdo AA, Fermi LAT Collaboration (2010a) Detection of Gamma-Ray Emission from the Starburst Galaxies M82 and NGC 253 with the Large Area Telescope on Fermi. Astrophys J Lett 709:L152-L157, DOI 10.1088/2041-8205/709/2/L152,0911.5327

Abdo AA, Ackermann M, Fermi LAT Collaboration (2010b) Observations of the Large Magellanic Cloud with Fermi. Astron Astrophys 512:A7, DOI 10.1051/0004-6361/200913474

Abramowski A, et al (2012a) Discovery of extended VHE $\gamma$-ray emission from the vicinity of the young massive stellar cluster Westerlund 1. Astron Astrophys 537:A114, DOI $10.1051 / 0004-6361 / 201117928,1111.2043$

Abramowski A, H E S S Collaboration (2012b) Spectral Analysis and Interpretation of the $\gamma$-Ray Emission from the Starburst Galaxy NGC 253. Astrophys J 757:158, DOI 10.1088/ 0004-637X/757/2/158, 1205.5485

Acero F, et al (2009) Detection of Gamma Rays from a Starburst Galaxy. Science 326:1080-, DOI 10.1126/science.1178826, 0909.4651

Ackermann M, et al (2011) A Cocoon of Freshly Accelerated Cosmic Rays Detected by Fermi in the Cygnus Superbubble. Science 334:1103-, DOI 10.1126/science.1210311

Ackermann M, et al (2012) GeV Observations of Star-forming Galaxies with the Fermi Large Area Telescope. Astrophys J 755:164, DOI 10.1088/0004-637X/755/2/164, 1206.1346

Aharonian F, et al HEGRA Collaboration (2002) An unidentified TeV source in the vicinity of Cygnus OB2. Astron Astrophys 393:L37-L40, DOI 10.1051/0004-6361:20021171, arXiv: astro-ph/0207528

Aharonian F, et al (2006) The H.E.S.S. Survey of the Inner Galaxy in Very High Energy Gamma Rays. Astrophys J 636:777-797, DOI 10.1086/498013, astro-ph/0510397

Aharonian F, et al (2007) Primary particle acceleration above $100 \mathrm{TeV}$ in the shell-type supernova remnant iASTROBJ ¿RX J1713.7-3946i/ASTROBJ $i$ with deep HESS observations. Astron Astrophys 464:235-243, DOI 10.1051/0004-6361:20066381, astro-ph/0611813

Aharonian FA (2004) Very high energy cosmic gamma radiation : a crucial window on the extreme Universe, River Edge, NJ: World Scientific Publishing

Albert J, et al (2008) MAGIC Observations of the Unidentified $\gamma$-Ray Source TeV J2032+4130. Astrophys J Lett 675:L25-L28, DOI 10.1086/529520, 0801.2391

Alexandrova O, Chen CHK, Sorriso-Valvo L, Horbury TS, Bale SD (2013) Solar Wind Turbulence and the Role of Ion Instabilities. Space Sci Rev 178:101-139, DOI 10.1007/ s11214-013-0004-8, 1306.5336

Alonso-Herrero A, Rieke GH, Rieke MJ, Kelly DM (2003) The [Fe II] 1.644 Micron Emission in M82 and NGC 253: Is It a Measure of the Supernova Rate? Astronom J 125:1210-1225, DOI $10.1086 / 367790$, astro-ph/0212142

Amato E (2014) The origin of galactic cosmic rays. International Journal of Modern Physics D 23:1430013, DOI 10.1142/S0218271814300134, 1406.7714

Amato E, Blasi P (2005) A general solution to non-linear particle acceleration at nonrelativistic shock waves. MNRAS 364:L76-L80, DOI 10.1111/j.1745-3933.2005.00110.x, arXiv:astro-ph/0509673

Anchordoqui LA, Paul TC, da Silva LHM, Torres DF, Vlcek BJ (2014) What IceCube data tell us about neutrino emission from star-forming galaxies (so far). Phys Rev D 89(12):127304, DOI 10.1103/PhysRevD.89.127304, 1405.7648

Anderson J, Piotto G, King IR, Bedin LR, Guhathakurta P (2009) Mixed Populations in Globular Clusters: Et Tu, 47 Tuc? Astrophys J Lett 697:L58-L62, DOI 10.1088/0004-637X/ $697 / 1 / \mathrm{L} 58,0904.1626$ 
Andrews JE, Calzetti D, Chandar R, Lee JC, Elmegreen BG, Kennicutt RC, Whitmore B, Kissel JS, da Silva RL, Krumholz MR, O'Connell RW, Dopita MA, Frogel JA, Kim H (2013) An Initial Mass Function Study of the Dwarf Starburst Galaxy NGC 4214. Astrophys J 767:51, DOI 10.1088/0004-637X/767/1/51, 1302.5006

Antecki T, Schlickeiser R, Zhang M (2013) Stochastic Acceleration of Suprathermal Particles under Pressure Balance Conditions. Astrophys J 764:89, DOI 10.1088/0004-637X/764/1/ 89

Apel WD, et al (2013) Separation of the light and heavy mass groups of $10^{16}-10^{18} \mathrm{eV}$ cosmic rays by studying the ratio muon size to shower size of KASCADE-Grande data. Journal of Physics Conference Series 409(1):012095, DOI 10.1088/1742-6596/409/1/012095

Armstrong JW, Rickett BJ, Spangler SR (1995) Electron density power spectrum in the local interstellar medium. Astrophys J 443:209-221, DOI 10.1086/175515

Arons J (2003) Magnetars in the Metagalaxy: An Origin for Ultra-High-Energy Cosmic Rays in the Nearby Universe. Astrophys J 589:871-892, DOI 10.1086/374776, arXiv:astro-ph/ 0208444

Artemyev AV, Hoshino M, Lutsenko VN, Petrukovich AA, Imada S, Zelenyi LM (2013) Double power-law spectra of energetic electrons in the Earth magnetotail. Annales Geophysicae 31:91-106, DOI 10.5194/angeo-31-91-2013

Ashour-Abdalla M, El-Alaoui M, Goldstein ML, Zhou M, Schriver D, Richard R, Walker R, Kivelson MG, Hwang KJ (2011) Observations and simulations of non-local acceleration of electrons in magnetotail magnetic reconnection events. Nature Physics 7:360-365, DOI $10.1038 /$ nphys 1903

Axford WI (1981) The acceleration of galactic cosmic rays. In: Setti G, Spada G, Wolfendale AW (eds) Origin of Cosmic Rays, IAU Symposium, vol 94, pp 339-358

Axford WI (1994) The origins of high-energy cosmic rays. Astrophys J Suppl 90:937-944, DOI 10.1086/191928

Bale SD, Kellogg PJ, Mozer FS, Horbury TS, Reme H (2005) Measurement of the Electric Fluctuation Spectrum of Magnetohydrodynamic Turbulence. Physical Review Letters 94(21):215002, DOI 10.1103/PhysRevLett.94.215002, physics/0503103

Bamba A, Ueno M, Nakajima H, Koyama K (2004) Thermal and Nonthermal X-Rays from the Large Magellanic Cloud Superbubble 30 Doradus C. Astrophys J 602:257-263, DOI 10.1086/380957, astro-ph/0310713

Bamba A, Yamazaki R, Yoshida T, Terasawa T, Koyama K (2005) A Spatial and Spectral Study of Nonthermal Filaments in Historical Supernova Remnants: Observational Results with Chandra. Astrophys J 621:793-802, DOI 10.1086/427620, astro-ph/0411326

Bastian N, Covey KR, Meyer MR (2010) A Universal Stellar Initial Mass Function? A Critical Look at Variations. Ann Rev Astron Astrophys 48:339-389, DOI 10.1146/ annurev-astro-082708-101642, 1001.2965

Batejat F, Conway JE, Hurley R, Parra R, Diamond PJ, Lonsdale CJ, Lonsdale CJ (2011) Resolution of the Compact Radio Continuum Sources in Arp220. Astrophys J 740:95, DOI 10.1088/0004-637X/740/2/95, 1109.6443

Baumgartner V, Breitschwerdt D (2013) Superbubble evolution in disk galaxies. I. Study of blow-out by analytical models. Astron Astrophys 557:A140, DOI 10.1051/0004-6361/ 201321261, 1402.0194

Beck R (2012) Magnetic Fields in Galaxies. Space SciRev 166:215-230, DOI 10.1007/ s11214-011-9782-z

Bednarek W (2005) GeV gamma-rays and TeV neutrinos from very massive compact binary systems: the case of WR 20a. Monthly Not Royal Astron Soc 363:L46-L50, DOI 10.1111/ j.1745-3933.2005.00081.x, astro-ph/0507565

Bell AR (1978) The acceleration of cosmic rays in shock fronts. I. MNRAS 182:147-156

Bell AR (2004) Turbulent amplification of magnetic field and diffusive shock acceleration of cosmic rays. MNRAS 353:550-558, DOI 10.1111/j.1365-2966.2004.08097.x

Bell AR (2013) Cosmic ray acceleration. Astroparticle Physics 43:56-70, DOI 10.1016/j. astropartphys.2012.05.022

Bell AR, Lucek SG (2001) Cosmic ray acceleration to very high energy through the nonlinear amplification by cosmic rays of the seed magnetic field. Monthly Not Royal Astron Soc 321:433-438, DOI 10.1046/j.1365-8711.2001.04063.x

Bell AR, Schure KM, Reville B, Giacinti G (2013) Cosmic-ray acceleration and escape from supernova remnants. Monthly Not Royal Astron Soc 431:415-429, DOI 10.1093/mnras/ stt179, 1301.7264 
Berezhko EG, Krymskii GF (1988) REVIEWS OF TOPICAL PROBLEMS: Acceleration of cosmic rays by shock waves. Soviet Physics Uspekhi 31:27-51, DOI 10.1070/ PU1988v031n01ABEH002534

Berezhnev SF, et al (2013) The Tunka - Multi-component EAS detector for high energy cosmic ray studies. Nuclear Instruments and Methods in Physics Research A 732:281-285, DOI 10.1016/j.nima.2013.05.180

Berezinskii VS, Bulanov SV, Dogiel VA, Ginzburg VL, Ptuskin VS (1990) Astrophysics of cosmic rays. Amsterdam: North-Holland

Bessho N, Bhattacharjee A (2012) Fast Magnetic Reconnection and Particle Acceleration in Relativistic Low-density Electron-Positron Plasmas without Guide Field. Astrophys J 750:129, DOI 10.1088/0004-637X/750/2/129

Biermann P (1976) On the radio continuum flux from the disks of spiral galaxies. Astron Astrophys 53:295-303

Biermann PL (1997) TOPICAL REVIEW: The origin of the highest energy cosmic rays. Journal of Physics G Nuclear Physics 23:1-27, DOI 10.1088/0954-3899/23/1/002

Binns WR, Wiedenbeck ME, Arnould M, Cummings AC, de Nolfo GA, Goriely S, Israel MH, Leske RA, Mewaldt RA, Stone EC, von Rosenvinge TT (2008) The OB association origin of galactic cosmic rays. New Astronomy Reviews 52:427-430, DOI 10.1016/j.newar.2008. 05.008

Birn J, Priest ER (2007) Reconnection of Magnetic Fields

Birn J, Thomsen MF, Hesse M (2004) Electron acceleration in the dynamic magnetotail: Test particle orbits in three-dimensional magnetohydrodynamic simulation fields. Physics of Plasmas 11:1825-1833, DOI 10.1063/1.1704641

Birn J, Artemyev AV, Baker DN, Echim M, Hoshino M, Zelenyi LM (2012) Particle Acceleration in the Magnetotail and Aurora. Space SciRev 173:49-102, DOI 10.1007/ s11214-012-9874-4

Biskamp D (2008) Magnetohydrodynamic Turbulence

Bisnovatyi-Kogan GS, Silich SA (1995) Shock-wave propagation in the nonuniform interstellar medium. Reviews of Modern Physics 67:661-712, DOI 10.1103/RevModPhys.67.661

Bland-Hawthorn J, Cohen M (2003) The Large-Scale Bipolar Wind in the Galactic Center. Astrophys J 582:246-256, DOI 10.1086/344573, astro-ph/0208553

Blandford R, Eichler D (1987) Particle acceleration at astrophysical shocks: A theory of cosmic ray origin. Phys Rep 154:1-75, DOI 10.1016/0370-1573(87)90134-7

Blasi P (2002) A semi-analytical approach to non-linear shock acceleration. Astroparticle Physics 16:429-439, DOI 10.1016/S0927-6505(01)00127-X, astro-ph/0104064

Blasi P (2013) The origin of galactic cosmic rays. Astron Astrophys Rev 21:70, DOI 10.1007/ s00159-013-0070-7, 1311.7346

Blasi P, Epstein RI, Olinto AV (2000) Ultra-High-Energy Cosmic Rays from Young Neutron Star Winds. Astrophys J Lett 533:L123-L126, DOI 10.1086/312626, astro-ph/9912240

Blasi P, Morlino G, Bandiera R, Amato E, Caprioli D (2012) Collisionless Shocks in a Partially Ionized Medium. I. Neutral Return Flux and its Effects on Acceleration of Test Particles. Astrophys J 755:121, DOI 10.1088/0004-637X/755/2/121, 1202.3080

Blitz L, Rosolowsky E (2006) The Role of Pressure in GMC Formation II: $\mathrm{The}_{2}$-Pressure Relation. Astrophys J 650:933-944, DOI 10.1086/505417, astro-ph/0605035

Bocchino F, Bykov AM, Chen Y, Krassilchtchikov AM, Levenfish KP, Miceli M, Pavlov GG, Uvarov YA, Zhou X (2012) A population of isolated hard X-ray sources near the supernova remnant Kes 69. Astron Astrophys 541:A152, DOI 10.1051/0004-6361/201219005, 1204. 3470

Bolatto AD, Warren SR, Leroy AK, Walter F, Veilleux S, Ostriker EC, Ott J, Zwaan M, Fisher DB, Weiss A, Rosolowsky E, Hodge J (2013) Suppression of star formation in the galaxy NGC 253 by a starburst-driven molecular wind. Nature 499:450-453, DOI $10.1038 /$ nature 12351

Bonnell IA, Larson RB, Zinnecker H (2007) The Origin of the Initial Mass Function. Protostars and Planets V pp 149-164, astro-ph/0603447

Booth CM, Agertz O, Kravtsov AV, Gnedin NY (2013) Simulations of Disk Galaxies with Cosmic Ray Driven Galactic Winds. Astrophys J Lett 777:L16, DOI 10.1088/2041-8205/ $777 / 1 /$ L16, 1308.4974

Brandenburg A, Nordlund $\AA$ (2011) Astrophysical turbulence modeling. Reports on Progress in Physics 74(4):046901, DOI 10.1088/0034-4885/74/4/046901, 0912.1340

Bromm V, Larson RB (2004) The First Stars. Ann Rev Astron Astrophys 42:79-118, DOI 
10.1146/annurev.astro.42.053102.134034, astro-ph/0311019

Bulanov SV, Sasorov PV (1976) Energy spectrum of particles accelerated in the neighborhood of a line of zero magnetic field. Soviet Astronomy Lett 19:464-468

Burgess D, Scholer M (2013) Microphysics of Quasi-parallel Shocks in Collisionless Plasmas. Space Sci Rev 178:513-533, DOI 10.1007/s11214-013-9969-6

Butt YM, Bykov AM (2008) A Cosmic-Ray Resolution to the Superbubble Energy Crisis. Astrophys J Lett 677:L21-L22, DOI 10.1086/587875, 0802.3805

Butt YM, Benaglia P, Combi JA, Corcoran M, Dame TM, Drake J, Kaufman Bernadó M, Milne P, Miniati F, Pohl M, Reimer O, Romero GE, Rupen M (2003) Chandra/Very Large Array Follow-Up of TeV J2032+4131, the Only Unidentified TeV Gamma-Ray Source. Astrophys J 597:494-512, DOI 10.1086/378121, astro-ph/0302342

Bykov AM (2001) Particle Acceleration and Nonthermal Phenomena in Superbubbles. Space Science Reviews 99:317-326

Bykov AM (2002) X-ray line emission from supernova ejecta fragments. Astron Astrophys 390:327-335, DOI 10.1051/0004-6361:20020694, arXiv:astro-ph/0205225

Bykov AM (2003) Faint hard X-ray sources in the Galactic Center region: Supernova ejecta fragments population. Astron Astrophys 410:L5-L8, DOI 10.1051/0004-6361:20031355, arXiv:astro-ph/0309234

Bykov AM, Fleishman GD (1992) On non-thermal particle generation in superbubbles. Monthly Not Royal Astron Soc 255:269-275

Bykov AM, Fleishman GD (2009) Particle Acceleration by Strong Turbulence in Solar Flares: Theory of Spectrum Evolution. Astrophys J Lett 692:L45-L49, DOI 10.1088/0004-637X/ 692/1/L45, 0901.2677

Bykov AM, Toptygin I (1993) Particle kinetics in highly turbulent plasmas (renormalization and self-consistent field methods). Physics Uspekhi 36:1020-1052, DOI 10.1070/ PU1993v036n11ABEH002179

Bykov AM, Toptygin IN (2001) A Model of Particle Acceleration to High Energies by Multiple Supernova Explosions in OB Associations. Astronomy Letters 27:625-633, DOI 10.1134/ 1.1404456

Bykov AM, Treumann RA (2011) Fundamentals of collisionless shocks for astrophysical application, 2. Relativistic shocks. Astron Astrophys Rev 19:42, DOI 10.1007/ s00159-011-0042-8, 1105.3221

Bykov AM, Chevalier RA, Ellison DC, Uvarov YA (2000) Nonthermal Emission from a Supernova Remnant in a Molecular Cloud. Astrphysical Journal 538:203-216, DOI 10.1086/309103, arXiv: astro-ph/0003235

Bykov AM, Dolag K, Durret F (2008) Cosmological Shock Waves. Space Sci Rev 134:119-140, DOI $10.1007 / \mathrm{s} 11214-008-9312-9,0801.0995$

Bykov AM, Ellison DC, Renaud M (2012) Magnetic Fields in Cosmic Particle Acceleration Sources. Space Sci Rev 166:71-95, DOI 10.1007/s11214-011-9761-4, 1105.0130

Bykov AM, Brandenburg A, Malkov MA, Osipov SM (2013a) Microphysics of Cosmic Ray Driven Plasma Instabilities. Space Sci Rev 178:201-232, DOI 10.1007/s11214-013-9988-3, 1304.7081

Bykov AM, Gladilin PE, Osipov SM (2013b) Non-linear model of particle acceleration at colliding shock flows. Monthly Not Royal Astron Soc 429:2755-2762, DOI 10.1093/mnras/ sts553, 1212.1556

Bykov AM, Malkov MA, Raymond JC, Krassilchtchikov AM, Vladimirov AE (2013c) Collisionless Shocks in Partly Ionized Plasma with Cosmic Rays: Microphysics of Non-thermal Components. Space Sci Rev 178:599-632, DOI 10.1007/s11214-013-9984-7, 1304.0998

Bykov AM, Ellison DC, Osipov SM, Vladimirov AE (2014) Magnetic Field Amplification in Nonlinear Diffusive Shock Acceleration Including Resonant and Non-resonant Cosmic-Ray Driven Instabilities. Astrophys J 789:137, DOI 10.1088/0004-637X/789/2/137, 1406.0084

Capelli R, Warwick RS, Porquet D, Gillessen S, Predehl P (2011) Fe K $\alpha$ line emission from the Arches cluster region - evidence for ongoing particle bombardment? Astron Astrophys 530:A38, DOI 10.1051/0004-6361/201116574, 1104.2039

Caprioli D, Spitkovsky A (2013) Cosmic-Ray-induced Filamentation Instability in Collisionless Shocks. Astrophys J Lett 765:L20, 1211.6765

Caprioli D, Amato E, Blasi P (2010) Non-linear diffusive shock acceleration with free-escape boundary. Astroparticle Physics 33:307-311, DOI 10.1016/j.astropartphys.2010.03.001, 0912.2714

Carretti E, Crocker RM, Staveley-Smith L, Haverkorn M, Purcell C, Gaensler BM, Bernardi 
G, Kesteven MJ, Poppi S (2013) Giant magnetized outflows from the centre of the Milky Way. Nature 493:66-69, DOI 10.1038/nature11734, 1301.0512

Casse M, Paul JA (1982) On the stellar origin of the Ne-22 excess in cosmic rays. Astrophys J 258:860-863, DOI 10.1086/160132

Castor J, McCray R, Weaver R (1975) Interstellar bubbles. Astrophys J Lett 200:L107-L110, DOI $10.1086 / 181908$

Cesarsky CJ, Montmerle T (1983) Gamma rays from active regions in the galaxy - The possible contribution of stellar winds. Space Sci Rev 36:173-193, DOI 10.1007/BF00167503

Chabrier G, Baraffe I (2000) Theory of Low-Mass Stars and Substellar Objects. Ann Rev Astron Astrophys 38:337-377, DOI 10.1146/annurev.astro.38.1.337, astro-ph/0006383

Chevalier RA (1999) Supernova Remnants in Molecular Clouds. Astrophys J 511:798-811, DOI $10.1086 / 306710$, astro-ph/9805315

Chevalier RA (2005) Young Core-Collapse Supernova Remnants and Their Supernovae. Astrophys J 619:839-855, DOI 10.1086/426584, astro-ph/0409013

Chevalier RA (2014) Supernova interaction with dense mass loss. In: Ray A, McCray RA (eds) IAU Symposium, IAU Symposium, vol 296, pp 95-102, DOI 10.1017/S1743921313009290, 1304.5500

Chevalier RA, Clegg AW (1985) Wind from a starburst galaxy nucleus. Nature 317:44, DOI $10.1038 / 317044 a 0$

Chiosi C, Maeder A (1986) The evolution of massive stars with mass loss. Ann Rev Astron Astrophys 24:329-375, DOI 10.1146/annurev.aa.24.090186.001553

Chu YH (2008) Bubbles and Superbubbles: Observations and Theory. In: Bresolin F, Crowther PA, Puls J (eds) IAU Symposium, IAU Symposium, vol 250, pp 341-354, DOI 10.1017/ S1743921308020681

Clark JS, Negueruela I, Ritchie B, Crowther P, Dougherty S (2010) Dissecting the Galactic Super Star Cluster Westerlund 1 - A Laboratory for Stellar Evolution. The Messenger 142:31-35

Combes F (1991) Distribution of CO in the Milky Way. Ann Rev Astron Astrophys 29:195-237, DOI 10.1146/annurev.aa.29.090191.001211

Condon JJ (1992) Radio emission from normal galaxies. Ann Rev Astron Astrophys 30:575611, DOI 10.1146/annurev.aa.30.090192.003043

Cram L, Hopkins A, Mobasher B, Rowan-Robinson M (1998) Star Formation Rates in Faint Radio Galaxies. Astrophys J 507:155-160, DOI 10.1086/306333, astro-ph/9805327

Crocker RM (2012) Non-thermal insights on mass and energy flows through the Galactic Centre and into the Fermi bubbles. Monthly Not Royal Astron Soc 423:3512-3539, DOI 10.1111/j.1365-2966.2012.21149.x, 1112.6247

Crowther PA, Schnurr O, Hirschi R, Yusof N, Parker RJ, Goodwin SP, Kassim HA (2010) The R136 star cluster hosts several stars whose individual masses greatly exceed the accepted $150 \mathrm{M}_{\text {solar }}$ stellar mass limit. Monthly Not Royal Astron Soc 408:731-751, DOI 10.1111/ j.1365-2966.2010.17167.x, 1007.3284

Damineli A, et al (2008) The periodicity of the $\eta$ Carinae events. Monthly Not Royal Astron Soc 384:1649-1656, DOI 10.1111/j.1365-2966.2007.12815.x, 0711.4250

De Becker M (2007) Non-thermal emission processes in massive binaries. Astron Astrophys Rev 14:171-216, DOI 10.1007/s00159-007-0005-2, 0709.4220

De Becker M, Raucq F (2013) Catalogue of particle-accelerating colliding-wind binaries. Astron Astrophys 558:A28, DOI 10.1051/0004-6361/201322074, 1308.3149

de Jong T, Klein U, Wielebinski R, Wunderlich E (1985) Radio continuum and far-infrared emission from spiral galaxies - A close correlation. Astron Astrophys 147:L6-L9

Dobrovolskas V, Kučinskas A, Bonifacio P, Korotin SA, Steffen M, Sbordone L, Caffau E, Ludwig HG, Royer F, Prakapavičius D (2014) Abundances of lithium, oxygen, and sodium in the turn-off stars of Galactic globular cluster 47 Tucanae. Astron Astrophys 565:A121, DOI 10.1051/0004-6361/201322868, 1311.1072

Dogiel V, Chernyshov D, Koyama K, Nobukawa M, Cheng KS (2011a) K-Shell Emission of Neutral Iron Line from Sagittarius B2 Excited by Subrelativistic Protons. Publ Astron Soc Japan 63:535-, DOI 10.1093/pasj/63.3.535, 1104.4484

Dogiel VA, Cheng KS, Chernyshov DO, Ip WH, Ko CM, Tatischeff V (2011b) X- and GammaRay Emission from the Galactic Center. In: Morris MR, Wang QD, Yuan F (eds) The Galactic Center: a Window to the Nuclear Environment of Disk Galaxies, Astronomical Society of the Pacific Conference Series, vol 439, p 426, 1002.1379

Dorfi EA, Breitschwerdt D (2012) Time-dependent galactic winds. I. Structure and evolution 
of galactic outflows accompanied by cosmic ray acceleration. Astron Astrophys 540:A77, DOI 10.1051/0004-6361/201118082, 1304.1311

Dougherty SM, Beasley AJ, Claussen MJ, Zauderer BA, Bolingbroke NJ (2005) HighResolution Radio Observations of the Colliding-Wind Binary WR 140. Astrophys J 623:447-459, DOI 10.1086/428494, astro-ph/0501391

Draine BT (2011) Physics of the Interstellar and Intergalactic Medium. Princeton University Press

Drake JF, Swisdak M, Che H, Shay MA (2006) Electron acceleration from contracting magnetic islands during reconnection. Nature 443:553-556, DOI 10.1038/nature05116

Drury LO (1983) An introduction to the theory of diffusive shock acceleration of energetic particles in tenuous plasmas. Reports on Progress in Physics 46:973-1027, DOI 10.1088/ 0034-4885/46/8/002

Dubus G (2013) Gamma-ray binaries and related systems. Astron Astrophys Rev 21:64, DOI $10.1007 / \mathrm{s} 00159-013-0064-5,1307.7083$

Dudok de Wit T, Alexandrova O, Furno I, Sorriso-Valvo L, Zimbardo G (2013) Methods for Characterising Microphysical Processes in Plasmas. Space Sci Rev 178:665-693, DOI $10.1007 / \mathrm{s} 11214-013-9974-9,1306.5303$

Durré M, Mould J (2014) Young Star Clusters In The Circumnuclear Region Of NGC 2110. ArXiv e-prints 1402.3339

Dwarkadas VV, Telezhinsky I, Pohl M (2012) On the maximum energy and escape of accelerated particles in young supernova remnants. In: Aharonian FA, Hofmann W, Rieger FM (eds) American Institute of Physics Conference Series, American Institute of Physics Conference Series, vol 1505, pp 245-248, DOI 10.1063/1.4772243

Dwek E, Krennrich F (2013) The extragalactic background light and the gamma-ray opacity of the universe. Astroparticle Physics 43:112-133, DOI 10.1016/j.astropartphys.2012.09.003, 1209.4661

Eichler D, Usov V (1993) Particle acceleration and nonthermal radio emission in binaries of early-type stars. Astrophys J 402:271-279, DOI 10.1086/172130

Ellison DC, Bykov AM (2011) Gamma-ray Emission of Accelerated Particles Escaping a Supernova Remnant in a Molecular Cloud. Astrophys J 731:87, DOI 10.1088/0004-637X/ $731 / 2 / 87,1102.3885$

Ellison DC, Slane P, Patnaude DJ, Bykov AM (2012) Core-collapse Model of Broadband Emission from SNR RX J1713.7-3946 with Thermal X-Rays and Gamma Rays from Escaping Cosmic Rays. Astrophys J 744:39, DOI 10.1088/0004-637X/744/1/39, 1109.0874

Elmegreen BG (2011a) Star Formation on Galactic Scales: Empirical Laws. In: Charbonnel C, Montmerle T (eds) EAS Publications Series, EAS Publications Series, vol 51, pp 3-17, DOI 10.1051 eas/1151001, 1101.3108

Elmegreen BG (2011b) Star Formation Patterns and Hierarchies. In: Charbonnel C, Montmerle T (eds) EAS Publications Series, EAS Publications Series, vol 51, pp 31-44, DOI 10.1051/ eas/1151003, 1101.3111

Elmegreen BG (2011c) Triggered Star Formation. In: Charbonnel C, Montmerle T (eds) EAS Publications Series, EAS Publications Series, vol 51, pp 45-58, DOI 10.1051/eas/1151004, 1101.3112

Elmegreen BG (2012) What triggers star formation in galaxies? In: Tuffs RJ, Popescu CC (eds) IAU Symposium, IAU Symposium, vol 284, pp 317-329, DOI 10.1017/S1743921312009350, 1201.3659

Elmegreen BG, Lada CJ (1977) Sequential formation of subgroups in OB associations. Astrophys J 214:725-741, DOI 10.1086/155302

Erlykin AD, Wolfendale AW (2012) A new component of cosmic rays? Astroparticle Physics 35:449-456, DOI 10.1016/j.astropartphys.2011.11.012

Farnier C, Walter R, Leyder JC (2011) $\eta$ Carinae: a very large hadron collider. Astron Astrophys 526:A57, DOI 10.1051/0004-6361/201015590

Federrath C, Sur S, Schleicher DRG, Banerjee R, Klessen RS (2011) A New Jeans Resolution Criterion for (M)HD Simulations of Self-gravitating Gas: Application to Magnetic Field Amplification by Gravity-driven Turbulence. Astrophys J 731:62, DOI 10.1088/0004-637X/731/1/62, 1102.0266

Feigelson ED, Townsley LK, Broos PS, Busk HA, Getman KV, King RR, Kuhn MA, Naylor T, Povich MS, Baddeley A, Bate MR, Indebetouw R, Luhman KL, McCaughrean MJ, Pittard JM, Pudritz RE, Sills A, Song Y, Wadsley J (2013) Overview of the Massive Young Star-Forming Complex Study in Infrared and X-Ray (MYStIX) Project. Astrophys 
J Suppl 209:26, DOI 10.1088/0067-0049/209/2/26, 1309.4483

Fermi E (1949) On the Origin of the Cosmic Radiation. Physical Review 75:1169-1174, DOI 10.1103/PhysRev.75.1169

Fermi E (1954) Galactic Magnetic Fields and the Origin of Cosmic Radiation. Astrophys J 119:1, DOI 10.1086/145789

Ferrand G, Marcowith A (2010) On the shape of the spectrum of cosmic rays accelerated inside superbubbles. Astron Astrophys 510:A101, DOI 10.1051/0004-6361/200913520,0911.4457

Ferrière K (2008) Large-scale distribution of interstellar matter in the central region of our Galaxy. Astronomische Nachrichten 329:992, DOI 10.1002/asna.200811054

Figer DF (2008) Young Massive Clusters. In: Bresolin F, Crowther PA, Puls J (eds) IAU Symposium, IAU Symposium, vol 250, pp 247-256, DOI 10.1017/S1743921308020565, 0801.4178

Figer DF, Najarro F, Gilmore D, Morris M, Kim SS, Serabyn E, McLean IS, Gilbert AM, Graham JR, Larkin JE, Levenson NA, Teplitz HI (2002) Massive Stars in the Arches Cluster. Astrophys J 581:258-275, DOI 10.1086/344154, astro-ph/0208145

Fisk LA, Gloeckler G (2006) The Common Spectrum for Accelerated Ions in the Quiet-Time Solar Wind. Astrophys J Lett 640:L79-L82, DOI 10.1086/503293

Fisk LA, Gloeckler G (2012) Particle Acceleration in the Heliosphere: Implications for Astrophysics. Space Sci Rev 173:433-458, DOI 10.1007/s11214-012-9899-8

Gabici S, Aharonian FA, Casanova S (2009) Broad-band non-thermal emission from molecular clouds illuminated by cosmic rays from nearby supernova remnants. Monthly Not Royal Astron Soc 396:1629-1639, DOI 10.1111/j.1365-2966.2009.14832.x, 0901.4549

Gargaté L, Spitkovsky A (2012) Ion Acceleration in Non-relativistic Astrophysical Shocks. Astrophys J 744:67, DOI 10.1088/0004-637X/744/1/67, 1107.0762

Georgy C, Walder R, Folini D, Bykov A, Marcowith A, Favre JM (2013) Circumstellar medium around rotating massive stars at solar metallicity. Astron Astrophys 559:A69, DOI 10. $1051 / 0004-6361 / 201321226,1309.1360$

Georgy C, Granada A, Ekström S, Meynet G, Anderson RI, Wyttenbach A, Eggenberger P, Maeder A (2014) Populations of rotating stars. III. SYCLIST, the new Geneva population synthesis code. Astron Astrophys 566:A21, DOI 10.1051/0004-6361/201423881, 1404.6952

Giacalone J, Burgess D, Schwartz SJ, Ellison DC, Bennett L (1997) Injection and acceleration of thermal protons at quasi-parallel shocks: A hybrid simulation parameter survey. J Geophys Res 102:19,789-19,804, DOI 10.1029/97JA01529

Ginzburg VL, Syrovatskii SI (1964) The Origin of Cosmic Rays. New York: Macmillan

Grigis PC, Benz AO (2006) Electron acceleration in solar flares: theory of spectral evolution. Astron Astrophys 458:641-651, DOI 10.1051/0004-6361:20065809, astro-ph/0606339

Groh JH, Nielsen KE, Damineli A, Gull TR, Madura TI, Hillier DJ, Teodoro M, Driebe T, Weigelt G, Hartman H, Kerber F, Okazaki AT, Owocki SP, Millour F, Murakawa K, Kraus S, Hofmann KH, Schertl D (2010) Detection of high-velocity material from the wind-wind collision zone of Eta Carinae across the 2009.0 periastron passage. Astron Astrophys 517:A9, DOI 10.1051/0004-6361/200913937, 1003.4527

Guesten R, Mezger PG (1982) Star formation and abundance gradients in the galaxy. Vistas in Astronomy 26:159-224, DOI 10.1016/0083-6656(82)90005-8

Guibert J, Lequeux J, Viallefond F (1978) Star formation in interstellar gas density in our Galaxy. Astron Astrophys 68:1-15

Hanasz M, Otmianowska-Mazur K, Kowal G, Lesch H (2009) Cosmic-ray-driven dynamo in galactic disks. A parameter study. Astron Astrophys 498:335-346, DOI 10.1051/ 0004-6361/200810279, 0812.3906

Harayama Y, Eisenhauer F, Martins F (2008) The Initial Mass Function of the Massive Starforming Region NGC 3603 from Near-Infrared Adaptive Optics Observations. Astrophys J 675:1319-1342, DOI 10.1086/524650, 0710.2882

Hartquist TW, Pittard JM, Falle SAEG (2007) Diffuse Matter from Star Forming Regions to Active Galaxies - A Volume Honouring John Dyson. DOI 10.1007/978-1-4020-5425-9

Heiles C (1990) Clustered supernovae versus the gaseous disk and halo. Astrophys J 354:483491, DOI 10.1086/168709

Heiles C (2001) The McKee/Ostriker Model: Paradigm? In: Woodward CE, Bicay MD, Shull JM (eds) Tetons 4: Galactic Structure, Stars and the Interstellar Medium, Astronomical Society of the Pacific Conference Series, vol 231, p 294, astro-ph/0010047

Heiles C, Haverkorn M (2012) Magnetic Fields in the Multiphase Interstellar Medium. Space Sci Rev 166:293-305, DOI 10.1007/s11214-012-9866-4 
Helder EA, Vink J, Bykov AM, Ohira Y, Raymond JC, Terrier R (2012) Observational Signatures of Particle Acceleration in Supernova Remnants. Space Sci Rev 173:369-431, DOI 10.1007/s11214-012-9919-8, 1206.1593

Helou G, Soifer BT, Rowan-Robinson M (1985) Thermal infrared and nonthermal radio Remarkable correlation in disks of galaxies. Astrophys J Lett 298:L7-L11, DOI 10.1086/ 184556

Hennebelle P, Falgarone E (2012) Turbulent molecular clouds. Astron Astrophys Rev 20:55, DOI 10.1007/s00159-012-0055-y, 1211.0637

Hennebelle P, Iffrig O (2014) Simulations of magnetized multiphase galactic disk regulated by supernovae explosions. ArXiv e-prints 1405.7819

HESS Collaboration, Abramowski A, et al HESS Collaboration (2011) Revisiting the Westerlund 2 field with the HESS telescope array. Astron Astrophys 525:A46, DOI 10.1051/ 0004-6361/201015290, 1009.3012

Higdon JC, Lingenfelter RE (2005) OB Associations, Supernova-generated Superbubbles, and the Source of Cosmic Rays. Astrophys J 628:738-749, DOI 10.1086/430814

Higdon JC, Lingenfelter RE, Ramaty R (1998) Cosmic-Ray Acceleration from Supernova Ejecta in Superbubbles. Astrophys J Lett 509:L33-L36, DOI 10.1086/311757

Hillas AM (2005) TOPICAL REVIEW: Can diffusive shock acceleration in supernova remnants account for high-energy galactic cosmic rays? Journal of Physics G Nuclear Physics 31:95, DOI 10.1088/0954-3899/31/5/R02

Hoshino M (2005) Electron surfing acceleration in magnetic reconnection. Journal of Geophysical Research (Space Physics) 110:A10215, DOI 10.1029/2005JA011229, astro-ph/0507528

Hoshino M (2012) Stochastic Particle Acceleration in Multiple Magnetic Islands during Reconnection. Physical Review Letters 108(13):135003, DOI 10.1103/PhysRevLett.108.135003, 1201.0837

Huang ZP, Thuan TX, Chevalier RA, Condon JJ, Yin QF (1994) Compact radio sources in the starburst galaxy M82 and the Sigma-D relation for supernova remnants. Astrophys J 424:114-125, DOI 10.1086/173876

IceCube Collaboration, Aartsen MG, Abbasi R, Abdou Y, Ackermann M, Adams J, Aguilar JA, Ahlers M, Altmann D, Auffenberg J, et al (2013a) Evidence for High-Energy Extraterrestrial Neutrinos at the IceCube Detector. Science 342:1242,856, DOI 10.1126/science. $1242856,1311.5238$

IceCube Collaboration, Abbasi R, et al (2013b) Cosmic ray composition and energy spectrum from 1-30 PeV using the 40-string configuration of IceTop and IceCube. Astroparticle Physics 42:15-32, DOI 10.1016/j.astropartphys.2012.11.003, 1207.3455

Jokipii JR, Lee MA (2010) Compression Acceleration in Astrophysical Plasmas and the Production of $\mathrm{f}(\mathrm{v})$ vprop $\mathrm{v}^{-5}$ Spectra in the Heliosphere. Astrophys J 713:475-483, DOI 10.1088/0004-637X/713/1/475

Jones FC (1994) A theoretical review of diffusive shock acceleration. Astrophys J Suppl 90:561565 , DOI $10.1086 / 191875$

Jones FC, Ellison DC (1991) The plasma physics of shock acceleration. Space Science Reviews 58:259-346, DOI 10.1007/BF01206003

Kamae T, Karlsson N, Mizuno T, Abe T, Koi T (2006) Parameterization of $\gamma, \mathrm{e}^{+/-}$, and Neutrino Spectra Produced by p-p Interaction in Astronomical Environments. ApJ 647:692708, DOI 10.1086/505189, arXiv: astro-ph/0605581

Karimabadi H, Roytershteyn V, Wan M, Matthaeus WH, Daughton W, Wu P, Shay M, Loring B, Borovsky J, Leonardis E, Chapman SC, Nakamura TKM (2013) Coherent structures, intermittent turbulence, and dissipation in high-temperature plasmas. Physics of Plasmas 20(1):012,303, DOI 10.1063/1.4773205

Kashiyama K, Mészáros P (2014) Galaxy Mergers as a Source of Cosmic Rays, Neutrinos, and Gamma Rays. Astrophys J Lett 790:L14, DOI 10.1088/2041-8205/790/1/L14, 1405.3262

Kato TN, Takabe H (2008) Nonrelativistic Collisionless Shocks in Unmagnetized Electron-Ion Plasmas. Astrophys J Lett 681:L93-L96, DOI 10.1086/590387, 0804.0052

Kato TN, Takabe H (2010) Nonrelativistic Collisionless Shocks in Weakly Magnetized ElectronIon Plasmas: Two-dimensional Particle-in-cell Simulation of Perpendicular Shock. Astrophys J 721:828-842, DOI 10.1088/0004-637X/721/1/828, 1008.0265

Kavanagh PJ, Norci L, Meurs EJA (2011) Diffuse thermal X-ray emission in the core of the young massive cluster Westerlund 1. New Astronomy 16:461-469, DOI 10.1016/j.newast. 2011.04.001, 1106.2665

Kavanagh PJ, Sasaki M, Bozzetto LM, Filipovic MD, Points SD, Maggi P, Haberl F (2014) 
XMM-Newton study of 30 Dor C and a newly identified MCSNR J0536-6913 in the Large Magellanic Cloud. ArXiv e-prints 1409.6547

Kelner SR, Aharonian FA, Bugayov VV (2006) Energy spectra of gamma rays, electrons, and neutrinos produced at proton-proton interactions in the very high energy regime. Physical Review D 74(3):034,018, DOI 10.1103/PhysRevD.74.034018, arXiv:astro-ph/0606058

Kennicutt RC, Evans NJ (2012) Star Formation in the Milky Way and Nearby Galaxies. Ann Rev Astron Astrophys 50:531-608, DOI 10.1146/annurev-astro-081811-125610, 1204.3552

Kennicutt RC Jr (1998a) Star Formation in Galaxies Along the Hubble Sequence. Ann Rev Astron Astrophys 36:189-232, DOI 10.1146/annurev.astro.36.1.189, astro-ph/9807187

Kennicutt RC Jr (1998b) The Global Schmidt Law in Star-forming Galaxies. Astrophys J 498:541-552, DOI 10.1086/305588, astro-ph/9712213

Kirk JG, Duffy P, Gallant YA (1996) Stochastic particle acceleration at shocks in the presence of braided magnetic fields. Astron Astrophys 314:1010-1016, astro-ph/9604056

Klepach EG, Ptuskin VS, Zirakashvili VN (2000) Cosmic ray acceleration by multiple spherical shocks. Astroparticle Physics 13:161-172, DOI 10.1016/S0927-6505(99)00108-5

Kolmogorov A (1941) The Local Structure of Turbulence in Incompressible Viscous Fluid for Very Large Reynolds' Numbers. Akademiia Nauk SSSR Doklady 30:301-305

Krause M, Fierlinger K, Diehl R, Burkert A, Voss R, Ziegler U (2013) Feedback by massive stars and the emergence of superbubbles. I. Energy efficiency and Vishniac instabilities. Astron Astrophys 550:A49, DOI 10.1051/0004-6361/201220060, 1207.7231

Krause M, Diehl R, Böhringer H, Freyberg M, Lubos D (2014) Feedback by massive stars and the emergence of superbubbles II. X-ray properties. ArXiv e-prints 1405.0037

Krivonos RA, et al (2014) First Hard X-Ray Detection of the Non-thermal Emission around the Arches Cluster: Morphology and Spectral Studies with NuSTAR. Astrophys J 781:107, DOI 10.1088/0004-637X/781/2/107,1312.2635

Kronberg PP, Biermann P, Schwab FR (1985) The nucleus of M82 at radio and X-ray bands - Discovery of a new radio population of supernova candidates. Astrophys J 291:693-707, DOI $10.1086 / 163108$

Kroupa P (2001) On the variation of the initial mass function. Monthly Not Royal Astron Soc 322:231-246, DOI 10.1046/j.1365-8711.2001.04022.x, astro-ph/0009005

Kroupa P (2002) The Initial Mass Function of Stars: Evidence for Uniformity in Variable Systems. Science 295:82-91, DOI 10.1126/science.1067524, astro-ph/0201098

Krumholz MR (2014) The Big Problems in Star Formation: the Star Formation Rate, Stellar Clustering, and the Initial Mass Function. ArXiv e-prints 1402.0867

Krumholz MR, McKee CF (2005) A General Theory of Turbulence-regulated Star Formation, from Spirals to Ultraluminous Infrared Galaxies. Astrophys J 630:250-268, DOI 10.1086/ 431734, astro-ph/0505177

Krumholz MR, Bate MR, Arce HG, Dale JE, Gutermuth R, Klein RI, Li ZY, Nakamura F, Zhang Q (2014) Star Cluster Formation and Feedback. ArXiv e-prints 1401.2473

Kudritzki RP, Puls J (2000) Winds from Hot Stars. Ann Rev Astron Astrophys 38:613-666, DOI 10.1146/annurev.astro.38.1.613

Lacki BC (2013) From 10K to 10 TK: Insights on the Interaction Between Cosmic Rays and Gas in Starbursts. In: Torres DF, Reimer O (eds) Cosmic Rays in Star-Forming Environments. Advances in Solid State Physics, vol 34, p 411, DOI 10.1007/978-3-642-35410-629, 1308. 5241

Lacki BC, Thompson TA, Quataert E, Loeb A, Waxman E (2011) On the GeV and TeV Detections of the Starburst Galaxies M82 and NGC 253. Astrophys J 734:107, DOI 10.1088/0004-637X/734/2/107, 1003.3257

Lada CJ, Lada EA (2003) Embedded Clusters in Molecular Clouds. Ann Rev Astron Astrophys 41:57-115, DOI 10.1146/annurev.astro.41.011802.094844, astro-ph/0301540

Lamers HJGLM, Cassinelli JP (1999) Introduction to Stellar Winds

Lang CC, Johnson KE, Goss WM, Rodríguez LF (2005) Stellar Winds and Embedded Star Formation in the Galactic Center Quintuplet and Arches Clusters: Multifrequency Radio Observations. Astronom J 130:2185-2196, DOI 10.1086/496976, astro-ph/0508178

Langer N (2013) Magnetic Fields in Stars: Origin and Impact. ArXiv e-prints 1312.2373

Law C, Yusef-Zadeh F (2004) X-Ray Observations of Stellar Clusters Near the Galactic Center. Astrophys J 611:858-870, DOI 10.1086/422307, astro-ph/0404544

Law CJ (2010) A Multiwavelength View of a Mass Outflow from the Galactic Center. Astrophys J 708:474-484, DOI 10.1088/0004-637X/708/1/474, 0911.2061

Lazarian A, Opher M (2009) A Model of Acceleration of Anomalous Cosmic Rays by Re- 
connection in the Heliosheath. Astrophys J 703:8-21, DOI 10.1088/0004-637X/703/1/8, 0905.1120

Leamon RJ, Smith CW, Ness NF, Matthaeus WH, Wong HK (1998) Observational constraints on the dynamics of the interplanetary magnetic field dissipation range. J Geophys Res 103:4775, DOI 10.1029/97JA03394

Lemoine M, Pelletier G (2010) On electromagnetic instabilities at ultra-relativistic shock waves. Monthly Not Royal Astron Soc 402:321-334, DOI 10.1111/j.1365-2966.2009.15869.x, 0904. 2657

Lemoine M, Kotera K, Pétri J (2014) On ultra-high energy cosmic ray acceleration at the termination shock of young pulsar winds. ArXiv e-prints 1409.0159

Lim B, Chun MY, Sung H, Park BG, Lee JJ, Sohn ST, Hur H, Bessell MS (2013) The Starburst Cluster Westerlund 1: The Initial Mass Function and Mass Segregation. DOI 10.1088/ 0004-6256/145/2/46, 1211.5832

Liu RY, Wang XY, Inoue S, Crocker R, Aharonian F (2014) Diffuse PeV neutrinos from EeV cosmic ray sources: Semirelativistic hypernova remnants in star-forming galaxies. Phys Rev D 89(8):083004, DOI 10.1103/PhysRevD.89.083004, 1310.1263

Loeb A, Waxman E (2006) The cumulative background of high energy neutrinos from starburst galaxies. J. Cosmol. Astropart. Phys. 5:003, DOI 10.1088/1475-7516/2006/05/003, astro-ph/0601695

Lyne AG, Manchester RN, Taylor JH (1985) The galactic population of pulsars. Monthly Not Royal Astron Soc 213:613-639

Mac Low MM, McCray R (1988) Superbubbles in disk galaxies. Astrophys J 324:776-785, DOI 10.1086/165936

Mackey J, Langer N, Meyer DMA, Gvaramadze VV, Mohamed S, Neilson HR, Mignone A (2014) The Circumstellar Medium of Massive Stars in Motion. ArXiv e-prints 1406.0878

Maddox LA, Williams RM, Dunne BC, Chu YH (2009) Nonthermal X-ray Emission in the N11 Superbubble in the Large Magellanic Cloud. Astrophys J 699:911-916, DOI 10.1088/ 0004-637X/699/1/911, 0904.1821

Madura TI, Gull TR, Okazaki AT, Russell CMP, Owocki SP, Groh JH, Corcoran MF, Hamaguchi K, Teodoro M (2013) Constraints on decreases in $\eta$ Carinae's mass-loss from 3D hydrodynamic simulations of its binary colliding winds. Monthly Not Royal Astron Soc 436:3820-3855, DOI 10.1093/mnras/stt1871, 1310.0487

Maeder A, Meynet G (1988) Tables of evolutionary star models from 0.85 to 120 solar masses with overshooting and mass loss. Astron Astrophys Suppl 76:411-425

Maeder A, Meynet G (2012) Rotating massive stars: From first stars to gamma ray bursts. Reviews of Modern Physics 84:25-63, DOI 10.1103/RevModPhys.84.25

Maeder A, Meynet G, Georgy C, Ekström S (2009) The basic role of magnetic fields in stellar evolution. In: Strassmeier KG, Kosovichev AG, Beckman JE (eds) IAU Symposium, IAU Symposium, vol 259, pp 311-322, DOI 10.1017/S1743921309030671, 0812.2764

Malkov MA (1997) Analytic Solution for Nonlinear Shock Acceleration in the Bohm Limit. Astrophysical Journal 485:638, DOI 10.1086/304471, arXiv:astro-ph/9707152

Malkov MA, Drury L (2001) Nonlinear theory of diffusive acceleration of particles by shock waves. Reports on Progress in Physics 64:429-481, DOI 10.1088/0034-4885/64/4/201

Mannheim K, Elsässer D, Tibolla O (2012) Gamma-rays from pulsar wind nebulae in starburst galaxies. Astroparticle Physics 35:797-800, DOI 10.1016/j.astropartphys.2012.02. 009, 1010.2185

Marcowith A, Parizot E, van der Swaluw E, Bykov A, Tatischeff V, Ferrand G (2005) Superbubbles: a laboratory for high energy astrophysics and cosmic-ray physics. In: Aharonian FA, Völk HJ, Horns D (eds) High Energy Gamma-Ray Astronomy, American Institute of Physics Conference Series, vol 745, pp 287-292, DOI 10.1063/1.1878418

Martin P (2014) Interstellar gamma-ray emission from cosmic rays in star-forming galaxies. Astron Astrophys 564:A61, DOI 10.1051/0004-6361/201323329, 1402.0383

Massey P (1998) The Initial Mass Function of Massive Stars in the Local Group. In: Gilmore G, Howell D (eds) The Stellar Initial Mass Function (38th Herstmonceux Conference), Astronomical Society of the Pacific Conference Series, vol 142, p 17

Massey P, Hunter DA (1998) Star Formation in R136: A Cluster of O3 Stars Revealed by Hubble Space Telescope Spectroscopy. Astrophys J 493:180-194, DOI 10.1086/305126

Matthaeus WH, Velli M (2011) Who Needs Turbulence?. A Review of Turbulence Effects in the Heliosphere and on the Fundamental Process of Reconnection. Space Sci Rev 160:145-168, DOI 10.1007/s11214-011-9793-9 
McClure-Griffiths NM, Green JA, Hill AS, Lockman FJ, Dickey JM, Gaensler BM, Green AJ (2013) Atomic Hydrogen in a Galactic Center Outflow. Astrophys J Lett 770:L4, DOI $10.1088 / 2041-8205 / 770 / 1 / \mathrm{L} 4,1304.7538$

McCray R, Kafatos M (1987) Supershells and propagating star formation. Astrophys J 317:190-196, DOI 10.1086/165267

McKee CF, Ostriker EC (2007) Theory of Star Formation. Ann Rev Astron Astrophys 45:565687, DOI 10.1146/annurev.astro.45.051806.110602, 0707.3514

Mereghetti S (2008) The strongest cosmic magnets: soft gamma-ray repeaters and anomalous X-ray pulsars. Astron Astrophys Rev 15:225-287, DOI 10.1007/s00159-008-0011-z, 0804. 0250

Meylan G, Heggie DC (1997) Internal dynamics of globular clusters. Astron Astrophys Rev 8:1143, DOI $10.1007 / \mathrm{s} 001590050008$, astro-ph/9610076

Molinari S, Bally J, Glover S, Moore T, Noriega-Crespo A, Plume R, Testi L, VázquezSemadeni E, Zavagno A, Bernard JP, Martin P (2014) The Milky Way as a Star Formation Engine. ArXiv e-prints 1402.6196

Montmerle T (1979) On gamma-ray sources, supernova remnants, OB associations, and the origin of cosmic rays. Astrophys J 231:95-110, DOI 10.1086/157166

Morris M, Serabyn E (1996) The Galactic Center Environment. Ann Rev Astron Astrophys 34:645-702, DOI 10.1146/annurev.astro.34.1.645

Muno MP, et al (2006a) A Neutron Star with a Massive Progenitor in Westerlund 1. Astrophys J Lett 636:L41-L44, DOI 10.1086/499776, astro-ph/0509408

Muno MP, Law C, Clark JS, Dougherty SM, de Grijs R, Portegies Zwart S, Yusef-Zadeh F (2006b) Diffuse, Nonthermal X-Ray Emission from the Galactic Star Cluster Westerlund 1. Astrophys J 650:203-211, DOI 10.1086/507175, astro-ph/0606492

Muno MP, et al (2009) A Catalog of X-Ray Point Sources from Two Megaseconds of Chandra Observations of the Galactic Center. Astrophys J Suppl 181:110-128, DOI 10.1088/0067-0049/181/1/110, 0809.1105

Norman CA, Ikeuchi S (1989) The disk-halo interaction - Superbubbles and the structure of the interstellar medium. Astrophys J 345:372-383, DOI 10.1086/167912

Ohm S (2012) $\gamma$-rays from starburst galaxies. In: Aharonian FA, Hofmann W, Rieger FM (eds) American Institute of Physics Conference Series, American Institute of Physics Conference Series, vol 1505, pp 64-71, DOI 10.1063/1.4772221, 1210.6888

Ohm S, Hinton JA, White R (2013) $\gamma$-ray emission from the Westerlund 1 region. Monthly Not Royal Astron Soc 434:2289-2294, DOI 10.1093/mnras/stt1170, 1306.5642

Oka M, Phan TD, Krucker S, Fujimoto M, Shinohara I (2010) Electron Acceleration by MultiIsland Coalescence. Astrophys J 714:915-926, DOI 10.1088/0004-637X/714/1/915, 1004. 1154

Ong RA, for the VERITAS Collaboration (2013) Recent VERITAS Results on VHE Gammaray Sources in Cygnus. ArXiv e-prints 1307.5003

Oskinova LM (2005) Evolution of X-ray emission from young massive star clusters. Monthly Not Royal Astron Soc 361:679-694, DOI 10.1111/j.1365-2966.2005.09229.x, astro-ph/ 0505512

Oskinova LM, Gruendl RA, Ignace R, Chu YH, Hamann WR, Feldmeier A (2010) Hard XRay Emission in the Star-Forming Region ON 2: Discovery with XMM-Newton. Astrophys J 712:763-777, DOI 10.1088/0004-637X/712/2/763, 1001.4798

Owocki SP (2014) Instabilities in the Envelopes and Winds of Very Massive Stars. ArXiv e-prints 1403.6745

Owocki SP, Castor JI, Rybicki GB (1988) Time-dependent models of radiatively driven stellar winds. I - Nonlinear evolution of instabilities for a pure absorption model. Astrophys J 335:914-930, DOI 10.1086/166977

Parizot E, Marcowith A, van der Swaluw E, Bykov AM, Tatischeff V (2004) Superbubbles and energetic particles in the Galaxy. I. Collective effects of particle acceleration. Astron Astrophys 424:747-760, DOI 10.1051/0004-6361:20041269, astro-ph/0405531

Parkin ER, Pittard JM (2008) A 3D dynamical model of the colliding winds in binary systems. Monthly Not Royal Astron Soc 388:1047-1061, DOI 10.1111/j.1365-2966.2008.13511.x, 0805.4529

Pedlar A, Wills KA (2007) The Messier 82 Starburst Galaxy, in book Diffuse Matter from Star Forming Regions to Active Galaxies, p 307

Pelletier G (2001) Fermi Acceleration of Astroparticles. In: Lemoine M, Sigl G (eds) Physics and Astrophysics of Ultra-High-Energy Cosmic Rays, Lecture Notes in Physics, Berlin 
Springer Verlag, vol 576, p 58

Petrosian V, Bykov AM (2008) Particle Acceleration Mechanisms. Space Sci Rev 134:207-227, DOI 10.1007/s11214-008-9315-6, 0801.0923

Pittard JM, Corcoran MF (2002) In hot pursuit of the hidden companion of eta Carinae: An X-ray determination of the wind parameters. Astron Astrophys 383:636-647, DOI 10.1051/0004-6361:20020025, astro-ph/0201105

Pittard JM, Dougherty SM (2006) Radio, X-ray, and $\gamma$-ray emission models of the collidingwind binary WR140. Monthly Not Royal Astron Soc 372:801-826, DOI 10.1111/j. 1365-2966.2006.10888.x, astro-ph/0603787

Portegies Zwart SF, McMillan SLW, Gieles M (2010) Young Massive Star Clusters. Ann Rev Astron Astrophys 48:431-493, DOI 10.1146/annurev-astro-081309-130834, 1002.1961

Prantzos N (2012) On the origin and composition of Galactic cosmic rays. Astron Astrophys 538:A80, DOI 10.1051/0004-6361/201117448, 1112.4343

Price DJ, Bate MR (2008) The effect of magnetic fields on star cluster formation. Monthly Not Royal Astron Soc 385:1820-1834, DOI 10.1111/j.1365-2966.2008.12976.x, 0801.3293

Price DJ, Bate MR (2009) Inefficient star formation: the combined effects of magnetic fields and radiative feedback. Monthly Not Royal Astron Soc 398:33-46, DOI 10.1111/j.1365-2966. 2009.14969.x, 0904.4071

Pritchett PL (2006) Relativistic electron production during guide field magnetic reconnection. Journal of Geophysical Research (Space Physics) 111:A10212, DOI 10.1029/2006JA011793

Ptuskin V, Zirakashvili V, Seo ES (2010) Spectrum of Galactic Cosmic Rays Accelerated in Supernova Remnants. Astrophys J 718:31-36, DOI 10.1088/0004-637X/718/1/31, 1006. 0034

Ptuskin VS, Zirakashvili VN (2005) On the spectrum of high-energy cosmic rays produced by supernova remnants in the presence of strong cosmic-ray streaming instability and wave dissipation. Astron Astrophys 429:755-765, DOI 10.1051/0004-6361:20041517, astro-ph/ 0408025

Puls J, Vink JS, Najarro F (2008) Mass loss from hot massive stars. Astron Astrophys Rev 16:209-325, DOI 10.1007/s00159-008-0015-8, 0811.0487

Ramaty R, Lingenfelter RE, Kozlovsky B (2001) Spallogenic Light Elements and Cosmic-ray Origin. Space Sci Rev 99:51-60

Rampadarath H, Morgan JS, Lenc E, Tingay SJ (2014) Multi-epoch Very Long Baseline Interferometric Observations of the Nuclear Starburst Region of NGC 253: Improved Modeling of the Supernova and Star formation Rates. Astronom J 147:5, DOI 10.1088/0004-6256/147/1/5, 1310.8033

Renaud F, Bournaud F, Kraljic K, Duc PA (2014) Starbursts triggered by intergalactic tides andinterstellar compressive turbulence. Monthly Not Royal Astron Soc 442:L33-L37, DOI $10.1093 / \mathrm{mnrasl} / \mathrm{slu} 050,1403.7316$

Ressler SM, Katsuda S, Reynolds SP, Long KS, Petre R, Williams BJ, Winkler PF (2014) Magnetic-Field Amplification in the Thin X-ray Rims of SN1006. ArXiv e-prints 1406.3630

Reville B, Kirk JG, Duffy P (2009) Steady-State Solutions in Nonlinear Diffusive Shock Acceleration. Astrophys J 694:951-958, DOI 10.1088/0004-637X/694/2/951, 0812.3993

Revnivtsev M, Sazonov S, Churazov E, Forman W, Vikhlinin A, Sunyaev R (2009) Discrete sources as the origin of the Galactic X-ray ridge emission. Nature 458:1142-1144, DOI 10.1038 /nature07946, 0904.4649

Revnivtsev MG, Churazov EM, Sazonov SY, Sunyaev RA, Lutovinov AA, Gilfanov MR, Vikhlinin AA, Shtykovsky PE, Pavlinsky MN (2004) Hard X-ray view of the past activity of $\mathrm{Sgr} \mathrm{A}^{*}$ in a natural Compton mirror. Astron Astrophys 425:L49-L52, DOI 10.1051/0004-6361:200400064, astro-ph/0408190

Reynolds SP, Gaensler BM, Bocchino F (2012) Magnetic Fields in Supernova Remnants and Pulsar-Wind Nebulae. Space Sci Rev 166:231-261, DOI 10.1007/s11214-011-9775-y, 1104. 4047

Rockefeller G, Fryer CL, Melia F, Wang QD (2005) Diffuse X-Rays from the Arches and Quintuplet Clusters. Astrophys J 623:171-180, DOI 10.1086/428605, astro-ph/0409704

Rogers H, Pittard JM (2013) Feedback from winds and supernovae in massive stellar clusters - I. Hydrodynamics. Monthly Not Royal Astron Soc 431:1337-1351, DOI 10.1093/mnras/ stt255, 1302.2443

Rogers H, Pittard JM (2014) Feedback from Winds and Supernovae in Massive Stellar Clusters - II. X-Ray Emission. ArXiv e-prints 1405.5115

Rosenberg MJF, van der Werf PP, Israel FP (2012) [FeII] as a tracer of supernova rate in nearby 
starburst galaxies. Astron Astrophys 540:A116, DOI 10.1051/0004-6361/201218772, 1202. 2713

Sagdeev RZ (1966) Cooperative Phenomena and Shock Waves in Collisionless Plasmas. Reviews of Plasma Physics 4:23

Sakamoto K, Wang J, Wiedner MC, Wang Z, Peck AB, Zhang Q, Petitpas GR, Ho PTP, Wilner DJ (2008) Submillimeter Array Imaging of the CO(3-2) Line and $860 \mu \mathrm{m}$ Continuum of Arp 220: Tracing the Spatial Distribution of Luminosity. Astrophys J 684:957-977, DOI $10.1086 / 590484,0806.0217$

Salem M, Bryan GL (2014) Cosmic ray driven outflows in global galaxy disc models. Monthly Not Royal Astron Soc 437:3312-3330, DOI 10.1093/mnras/stt2121

Salpeter EE (1955) The Luminosity Function and Stellar Evolution. Astrophys J 121:161, DOI $10.1086 / 145971$

Sanchez Almeida J, Elmegreen BG, Munoz-Tunon C, Elmegreen DM (2014) Star formation sustained by gas accretion. ArXiv e-prints 1405.3178

Scalo JM (1986) The stellar initial mass function. Fundamentals of Cosmic Physics 11:1-278

Schekochihin AA, Cowley SC, Dorland W, Hammett GW, Howes GG, Quataert E, Tatsuno T (2009) Astrophysical Gyrokinetics: Kinetic and Fluid Turbulent Cascades in Magnetized Weakly Collisional Plasmas. Astrophys J Suppl 182:310-377, DOI 10.1088/0067-0049/ $182 / 1 / 310,0704.0044$

Schleicher DRG, Beck R (2013) A new interpretation of the far-infrared - radio correlation and the expected breakdown at high redshift. Astron Astrophys 556:A142, DOI 10.1051/ 0004-6361/201321707, 1306.6652

Schneider FRN, Izzard RG, de Mink SE, Langer N, Stolte A, de Koter A, Gvaramadze VV, Hußmann B, Liermann A, Sana H (2014) Ages of Young Star Clusters, Massive Blue Stragglers, and the Upper Mass Limit of Stars: Analyzing Age-dependent Stellar Mass Functions. Astrophys J 780:117, DOI 10.1088/0004-637X/780/2/117, 1312.0607

Schure KM, Bell AR, O'C Drury L, Bykov AM (2012) Diffusive Shock Acceleration and Magnetic Field Amplification. Space Sci Rev DOI 10.1007/s11214-012-9871-7, 1203.1637

Servidio S, Dmitruk P, Greco A, Wan M, Donato S, Cassak PA, Shay MA, Carbone V, Matthaeus WH (2011) Magnetic reconnection as an element of turbulence. Nonlinear Processes in Geophysics 18:675-695, DOI 10.5194/npg-18-675-2011

Siejkowski H, Otmianowska-Mazur K, Soida M, Bomans DJ, Hanasz M (2014) 3D global simulations of a cosmic-ray-driven dynamo in dwarf galaxies. Astron Astrophys 562:A136, DOI 10.1051/0004-6361/201220367, 1401.5293

Sironi L, Spitkovsky A (2012) Particle-in-cell simulations of shock-driven reconnection in relativistic striped winds. Computational Science and Discovery 5(1):014014, DOI 10.1088/ $1749-4699 / 5 / 1 / 014014,1208.4998$

Smith HE, Lonsdale CJ, Lonsdale CJ, Diamond PJ (1998) A Starburst Revealed-Luminous Radio Supernovae in the Nuclei of ARP 220. Astrophys J Lett 493:L17-L21, DOI 10.1086/ 311122

Socrates A, Davis SW, Ramirez-Ruiz E (2008) The Eddington Limit in Cosmic Rays: An Explanation for the Observed Faintness of Starbursting Galaxies. Astrophys J 687:202215, DOI 10.1086/590046, astro-ph/0609796

Stevens IR, Blondin JM, Pollock AMT (1992) Colliding winds from early-type stars in binary systems. Astrophys J 386:265-287, DOI 10.1086/171013

Strong AW, Moskalenko IV, Ptuskin VS (2007) Cosmic-Ray Propagation and Interactions in the Galaxy. Annual Review of Nuclear and Particle Science 57:285-327, DOI 10.1146/ annurev.nucl.57.090506.123011, astro-ph/0701517

Su M, Slatyer TR, Finkbeiner DP (2010) Giant Gamma-ray Bubbles from Fermi-LAT: Active Galactic Nucleus Activity or Bipolar Galactic Wind? Astrophys J 724:1044-1082, DOI 10.1088/0004-637X/724/2/1044, 1005.5480

Sunyaev R, Churazov E (1998) Equivalent width, shape and proper motion of the iron fluorescent line emission from molecular clouds as an indicator of the illuminating source X-ray flux history. Monthly Not Royal Astron Soc 297:1279-1291, DOI 10.1046/j.1365-8711.1998. 01684.x, astro-ph/9805038

Sunyaev RA, Markevitch M, Pavlinsky M (1993) The center of the Galaxy in the recent past - A view from GRANAT. Astrophys J 407:606-610, DOI 10.1086/172542

Tamborra I, Ando S, Murase K (2014) Star-forming galaxies as the origin of diffuse high-energy backgrounds: Gamma-ray and neutrino connections, and implications for starburst history. Journal of Cosmology and Astroparticle Physics 2014(09):043, astro-ph/1404.1189 
Tan JC, Beltran MT, Caselli P, Fontani F, Fuente A, Krumholz MR, McKee CF, Stolte A (2014) Massive Star Formation. ArXiv e-prints 1402.0919

Tananbaum H, Weisskopf M, Tucker W, Wilkes B, Edmonds P (2014) Highlights and Discoveries from the Chandra X-ray Observatory. ArXiv e-prints 1405.7847

Tatischeff V, Decourchelle A, Maurin G (2012) Nonthermal X-rays from low-energy cosmic rays: application to the $6.4 \mathrm{keV}$ line emission from the Arches cluster region. Astron Astrophys 546:A88, DOI 10.1051/0004-6361/201219016, 1210.2108

Tavani M, et al (2009) Detection of Gamma-Ray Emission from the Eta-Carinae Region. Astrophys J Lett 698:L142-L146, DOI 10.1088/0004-637X/698/2/L142, 0904.2736

Terrier R, Ponti G, Bélanger G, Decourchelle A, Tatischeff V, Goldwurm A, Trap G, Morris MR, Warwick R (2010) Fading Hard X-ray Emission from the Galactic Center Molecular Cloud Sgr B2. Astrophys J 719:143-150, DOI 10.1088/0004-637X/719/1/143, 1005.4807

The ARGO-YBJ Collaboration, :, Bartoli B, et al (2014) Identification of the TeV Gamma-ray Source ARGO J2031+4157 with the Cygnus Cocoon. ArXiv e-prints 1406.6436

Thompson MA, Urquhart JS, Moore TJT, Morgan LK (2012) The statistics of triggered star formation: an overdensity of massive young stellar objects around Spitzer bubbles. Monthly Not Royal Astron Soc 421:408-418, DOI 10.1111/j.1365-2966.2011.20315.x, 1111.0972

Tidman DA, Krall NA (1971) Shock waves in collisionless plasmas

Toptygin IN (1985) Cosmic rays in interplanetary magnetic fields. D. Reidel Publishing Co.

Torres DF, Reimer O (eds) (2013) Cosmic Rays in Star-Forming Environments, Advances in Solid State Physics, vol 34, DOI 10.1007/978-3-642-35410-6

Treumann RA (2009) Fundamentals of collisionless shocks for astrophysical application, 1 . Nonrelativistic shocks. Astron Astrophys Rev 17:409-535, DOI 10.1007/s00159-009-0024-2

Tu CY, Marsch E (1995) MHD structures, waves and turbulence in the solar wind: Observations and theories. Space Sci Rev 73:1-210, DOI 10.1007/BF00748891

Tutukov AV (1978) Early Stages of Dynamical Evolution of Star Cluster Models. Astron Astrophys 70:57

Uchiyama Y, Aharonian FA, Tanaka T, Takahashi T, Maeda Y (2007) Extremely fast acceleration of cosmic rays in a supernova remnant. Nature 449:576-578, DOI 10.1038/nature06210

Unger SW, Pedlar A, Axon DJ, Wilkinson PN, Appleton PN (1984) Young supernovae in the starbust galaxy M82. Monthly Not Royal Astron Soc 211:783-792

Valinia A, Tatischeff V, Arnaud K, Ebisawa K, Ramaty R (2000) On the Origin of the Iron $\mathrm{K}$ Line in the Spectrum of The Galactic X-Ray Background. Astrophys J 543:733-739, DOI 10.1086/317133, astro-ph/0006202

van Marle AJ, Meliani Z, Marcowith A (2012) A hydrodynamical model of the circumstellar bubble created by two massive stars. Astron Astrophys 541:L8, DOI 10.1051/0004-6361/ 201219180, 1204.2078

Veilleux S, Cecil G, Bland-Hawthorn J (2005) Galactic Winds. Ann Rev Astron Astrophys 43:769-826, DOI 10.1146/annurev.astro.43.072103.150610, astro-ph/0504435

Velázquez PF, Koenigsberger G, Raga AC (2003) A Supernova Remnant Collision with a Stellar Wind. Astrophys J 584:284-292, DOI 10.1086/345663, astro-ph/0211491

Vink J (2012) Supernova remnants: the X-ray perspective. Astron Astrophys Rev 20:49, DOI $10.1007 / \mathrm{s} 00159-011-0049-1,1112.0576$

Vink J, Kuiper L (2006) Supernova remnant energetics and magnetars: no evidence in favour of millisecond proto-neutron stars. Monthly Not Royal Astron Soc 370:L14-L18, DOI 10.1111/j.1745-3933.2006.00178.x, astro-ph/0604187

Vink JS, de Koter A, Lamers HJGLM (2000) New theoretical mass-loss rates of O and B stars. Astron Astrophys 362:295-309, astro-ph/0008183

Vink JS, de Koter A, Lamers HJGLM (2001) Mass-loss predictions for O and B stars as a function of metallicity. Astron Astrophys 369:574-588, DOI 10.1051/0004-6361:20010127, astro-ph/0101509

Voelk HJ (1989) The correlation between radio and far-infrared emission for disk galaxies - A calorimeter theory. Astron Astrophys 218:67-70

Voelk HJ, Biermann PL (1988) Maximum energy of cosmic-ray particles accelerated by supernova remnant shocks in stellar wind cavities. Astrophys J Lett 333:L65-L68, DOI $10.1086 / 185289$

Walder R, Folini D, Meynet G (2012) Magnetic Fields in Massive Stars, Their Winds, and Their Nebulae. Space Sci Rev 166:145-185, DOI 10.1007/s11214-011-9771-2, 1103.3777

Wang QD (2014) Supernovae and the Galactic Ecosystem. In: Ray A, McCray RA (eds) IAU Symposium, IAU Symposium, vol 296, pp 273-281, DOI 10.1017/S1743921313009587, 
1401.6209

Wang QD, Dong H, Lang C (2006) The interplay between star formation and the nuclear environment of our Galaxy: deep X-ray observations of the Galactic centre Arches and Quintuplet clusters. Monthly Not Royal Astron Soc 371:38-54, DOI 10.1111/j.1365-2966. 2006.10656.x, astro-ph/0606282

Weaver R, McCray R, Castor J, Shapiro P, Moore R (1977) Interstellar bubbles. II - Structure and evolution. Astrophys J 218:377-395, DOI 10.1086/155692

Weidner C, Bonnell IA, Zinnecker H (2010a) Super Star Clusters Versus OB Associations. Astrophys J 724:1503-1508, DOI 10.1088/0004-637X/724/2/1503, 1009.1618

Weidner C, Kroupa P, Bonnell IAD (2010b) The relation between the most-massive star and its parental star cluster mass. Monthly Not Royal Astron Soc 401:275-293, DOI 10.1111/ j.1365-2966.2009.15633.x, 0909.1555

Weidner C, Kroupa P, Pflamm-Altenburg J (2011) Top-heavy integrated galactic stellar initial mass functions in starbursts. Monthly Not Royal Astron Soc 412:979-986, DOI 10.1111/ j.1365-2966.2010.17959.x, 1011.3814

Weidner C, Kroupa P, Pflamm-Altenburg J (2014) Sampling methods for stellar masses and the mmax-Mecl relation in the starburst dwarf galaxy NGC 4214. ArXiv e-prints 1404.1075

Werner M, Reimer O, Reimer A, Egberts K (2013) Fermi-LAT upper limits on gamma-ray emission from colliding wind binaries. Astron Astrophys 555:A102, DOI 10.1051/0004-6361/ $201220502,1308.2573$

Whitmore BC, Schweizer F (1995) Hubble space telescope observations of young star clusters in NGC-4038/4039, 'the antennae' galaxies. Astronom J 109:960-980, DOI 10.1086/117334

Whitmore BC, Chandar R, Schweizer F, Rothberg B, Leitherer C, Rieke M, Rieke G, Blair WP, Mengel S, Alonso-Herrero A (2010) The Antennae Galaxies (NGC 4038/4039) Revisited: Advanced Camera for Surveys and NICMOS Observations of a Prototypical Merger. Astronom J 140:75-109, DOI 10.1088/0004-6256/140/1/75, 1005.0629

Winske D, Quest KB (1988) Magnetic field and density fluctuations at perpendicular supercritical collisionless shocks. J Geophys Res 93:9681-9693, DOI 10.1029/JA093iA09p09681

Winske D, Thomas VA, Omidi N, Quest KB (1990) Re-forming supercritical quasiparallel shocks. II - Mechanism for wave generation and front re-formation. J Geophys Res 95:18,821-18,832, DOI 10.1029/JA095iA11p18821

Wright NJ, Parker RJ, Goodwin SP, Drake JJ (2014) Constraints on massive star formation: Cygnus OB2 was always an association. Monthly Not Royal Astron Soc 438:639-646, DOI $10.1093 / \mathrm{mnras} / \mathrm{stt} 2232,1311.4537$

Wu EMH, Hui CY, Kong AKH, Tam PHT, Cheng KS, Dogiel VA (2014) Chandra detection of a new diffuse X-ray component from the globular cluster 47 Tucanae. Astrophys J Lett 788(2):L40, URL http://stacks .iop.org/2041-8205/788/i=2/a=L40 1405.5481

Yoast-Hull TM, Gallagher JS III, Zweibel EG, Everett JE (2014) Active Galactic Nuclei, Neutrinos, and Interacting Cosmic Rays in NGC 253 and NGC 1068. Astrophys J 780:137, DOI 10.1088/0004-637X/780/2/137, 1311.5586

Yusef-Zadeh F (2003) The Origin of the Galactic Center Nonthermal Radio Filaments: Young Stellar Clusters. Astrophys J 598:325-333, DOI 10.1086/378715, astro-ph/0308008

Yusef-Zadeh F, Wardle M, Lis D, Viti S, Brogan C, Chambers E, Pound M, Rickert M (2013) 74 $\mathrm{MHz}$ Nonthermal Emission from Molecular Clouds: Evidence for a Cosmic Ray Dominated Region at the Galactic Center. Journal of Physical Chemistry A 117:9404-9419, DOI $10.1021 / \mathrm{jp} 311240 \mathrm{~h}, 1305.1047$

Zelenyi LM, Lominadze JG, Taktakishvili AL (1990) Generation of the energetic proton and electron bursts in planetary magnetotails. J Geophys Res 95:3883-3891, DOI 10.1029/ JA095iA04p03883

Zhang M, Lee MA (2013) Stochastic Acceleration of Energetic Particles in the Heliosphere. Space Sci Rev 176:133-146, DOI 10.1007/s11214-011-9754-3

Zharkova VV, et al (2011) Recent Advances in Understanding Particle Acceleration Processes in Solar Flares. Space Sci Rev 159:357-420, DOI 10.1007/s11214-011-9803-y, 1110.2359

Zimbardo G, Perri S (2013) From Lévy Walks to Superdiffusive Shock Acceleration. Astrophys J 778:35, DOI 10.1088/0004-637X/778/1/35

Zinnecker H, Yorke HW (2007) Toward Understanding Massive Star Formation. Ann Rev Astron Astrophys 45:481-563, DOI 10.1146/annurev.astro.44.051905.092549, 0707.1279

Zweibel E (2013) Astrophysics: Recipe for regularity. Nature 502:453-454, DOI 10.1038/ $502453 \mathrm{a}$

Zweibel EG (2003) Cosmic-Ray History and Its Implications for Galactic Magnetic Fields. 
Astrophys J 587:625-637, DOI 10.1086/368256, astro-ph/0212559

Zweibel EG, Everett JE (2010) Environments for Magnetic Field Amplification by Cosmic Rays. Astrophys J 709:1412-1419, DOI 10.1088/0004-637X/709/2/1412, 0912.3511

Zweibel EG, Yamada M (2009) Magnetic Reconnection in Astrophysical and Laboratory Plasmas. Ann Rev Astron Astrophys 47:291-332, DOI 10.1146/annurev-astro-082708-101726 